\title{
Myeloperoxidase and anti-myeloperoxidase autoantibodies in renal inflammation
}

Citation for published version (APA):

Huugen, D. (2007). Myeloperoxidase and anti-myeloperoxidase autoantibodies in renal inflammation. [Doctoral Thesis, Maastricht University]. Universiteit Maastricht. https://doi.org/10.26481/dis.20071005dh

Document status and date:

Published: 01/01/2007

DOI:

$10.26481 /$ dis.20071005dh

Document Version:

Publisher's PDF, also known as Version of record

\section{Please check the document version of this publication:}

- A submitted manuscript is the version of the article upon submission and before peer-review. There can be important differences between the submitted version and the official published version of record.

People interested in the research are advised to contact the author for the final version of the publication, or visit the DOI to the publisher's website.

- The final author version and the galley proof are versions of the publication after peer review.

- The final published version features the final layout of the paper including the volume, issue and page numbers.

Link to publication

\footnotetext{
General rights rights.

- You may freely distribute the URL identifying the publication in the public portal. please follow below link for the End User Agreement:

www.umlib.nl/taverne-license

Take down policy

If you believe that this document breaches copyright please contact us at:

repository@maastrichtuniversity.nl

providing details and we will investigate your claim.
}

Copyright and moral rights for the publications made accessible in the public portal are retained by the authors and/or other copyright owners and it is a condition of accessing publications that users recognise and abide by the legal requirements associated with these

- Users may download and print one copy of any publication from the public portal for the purpose of private study or research.

- You may not further distribute the material or use it for any profit-making activity or commercial gain

If the publication is distributed under the terms of Article $25 \mathrm{fa}$ of the Dutch Copyright Act, indicated by the "Taverne" license above, 
Myeloperoxidase and anti-myeloperoxidase autoantibodies in renal inflammation

Dennis Huugen 
Financial support by the Dutch Kidney Foundation (Nierstichting), the Wegener's Foundation (Friedrich Wegener Stichting) and the J.E. Jurriaanse Stichting is gratefully acknowledged.

In addition, printing of this thesis was financially supported by Baxter, Boehringer Ingelheim, and Novartis Pharma

(C) D. Huugen, Maastricht 2007

ISBN 978-90-9022189-2

Printed by Gildeprint BV, Enschede 


\title{
Myeloperoxidase and anti-myeloperoxidase autoantibodies in renal inflammation
}

\author{
PROEFSCHRIFT
}

Ter verkrijging van de graad van doctor aan de Universiteit Maastricht, op gezag van de Rector Magnificus, Prof. mr. G.P.M.F. Mols volgens het besluit van het College van Decanen, in het openbaar te verdedigen op vrijdag 5 oktober 2007 om 14.00 uur

door

Dennis Huugen 


\section{Promotor:}

Prof. Dr. J.W. Cohen Tervaert

\section{Copromotor:}

Dr. P. Heeringa (Universitair Medisch Centrum Groningen)

\section{Beoordelingscommissie:}

Prof. Dr. M. Daha (Universiteit Leiden)

Dr. R. Goldschmeding (Universiteit Utrecht)

Prof. Dr. F. J. van Schooten

Prof. Dr. H. Schouten

Prof. Dr. E. Wouters (voorzitter) 




\section{Table of contents}

Table of contents.

Chapter 1 - General introduction.

Chapter 2 - Aggravation of anti-myeloperoxidase antibody-induced glomerulonephritis by bacterial lipopolysaccharide: role of tumor necrosis factor $\alpha$

Chapter 3 - Tumor necrosis factor-alpha bioactivity-inhibiting therapy in ANCA-

associated vasculitis - clinical and experimental considerations.

Chapter 4 - Inhibition of complement factor C5 protects against anti-myeloperoxidase antibody-mediated crescentic glomerulonephritis in mice

Chapter 5 - Monoclonal antibodies specific for murine myeloperoxidase aggravate mild anti-glomerular basement membrane (GBM) antibody-induced glomerulonephritis .77

Chapter $6-$ MPO is critically involved in the induction of organ damage after renal ischemia reperfusion

Chapter 7 - Summary and discussion

References

Nederlandse samenvatting

Dankwoord

Curriculum vitae

Publications

Appendix with full color pictures 



\section{Chapter 1 - General introduction}

In part published as:

Huugen D, Tervaert JW, Heeringa P. Antineutrophil cytoplasmic autoantibodies and pathophysiology: new insights from animal models. Curr Opin Rheumatol. 2004 Jan;16(1):4-8. 


\section{The systemic vasculitides}

Vasculitides are "chronic inflammatory diseases in which blood vessels are targeted by an immune insult" [1]. They can occur as primary disease (primary vasculitis), but also in response to several drugs, or in the context of an underlying disease, such as various malignancies, myelodysplasia, and several bacterial and viral infections (secondary vasculitis).

Historically, the classification of vasculitides is predominantly based upon histopathological and immunohistochemical characteristics, such as the size of the vessels that are involved, and the presence or absence of immune-complexes. Until 1994, it was very difficult to obtain a uniform, widely accepted, classification system. In 1994 however, a classification scheme for systemic idiopathic (primary) vasculitides was presented as a result of the Chapel Hill Consensus Conference [2]. This classification scheme is now widely used, and importantly facilitates the diagnosis, treatment as well as research in this category of diseases. In Figure 1-1, the primary systemic vasculitides are arranged according to their main site of occurrence in the vascular system, as proposed by the Chapel Hill Consensus conference. This thesis focuses on a subgroup of small vessel vasculitides that is associated with the presence of anti-neutrophil cytoplasmic autoantibodies (ANCA).



Figure 1-1: Preferred sites of vascular involvement by selected vasculitides. The widths of the trapezoids indicate the frequencies of involvement of various portions of the vasculature. LCA: leukocytoclastic angiitis (adapted from Jennette et al., 1997 [3]) 


\section{ANCA-associated vasculitis}

Wegener's Granulomatosis (WG), Churg-Strauss Syndrome (CSS) microscopic polyangiitis (MPA), and idiopathic necrotizing crescentic glomerulonephritis (NCGN) have several characteristics in common. First, their diagnosis is based histopathologically on the presence of destructive inflammation of the arterioles, capillaries and postcapillary venules without extensive depositions of immune components such as complement and immunoglobulins (pauci-immune). Second, they are often accompanied by elevated levels of anti-neutrophil cytoplasmic autoantibodies (ANCA), predominantly specific for proteinase 3 (PR3-ANCA) and myeloperoxidase (MPO-ANCA). Therefore, they are collectively referred to as ANCA-associated small-vessel vasculitides [2-6].
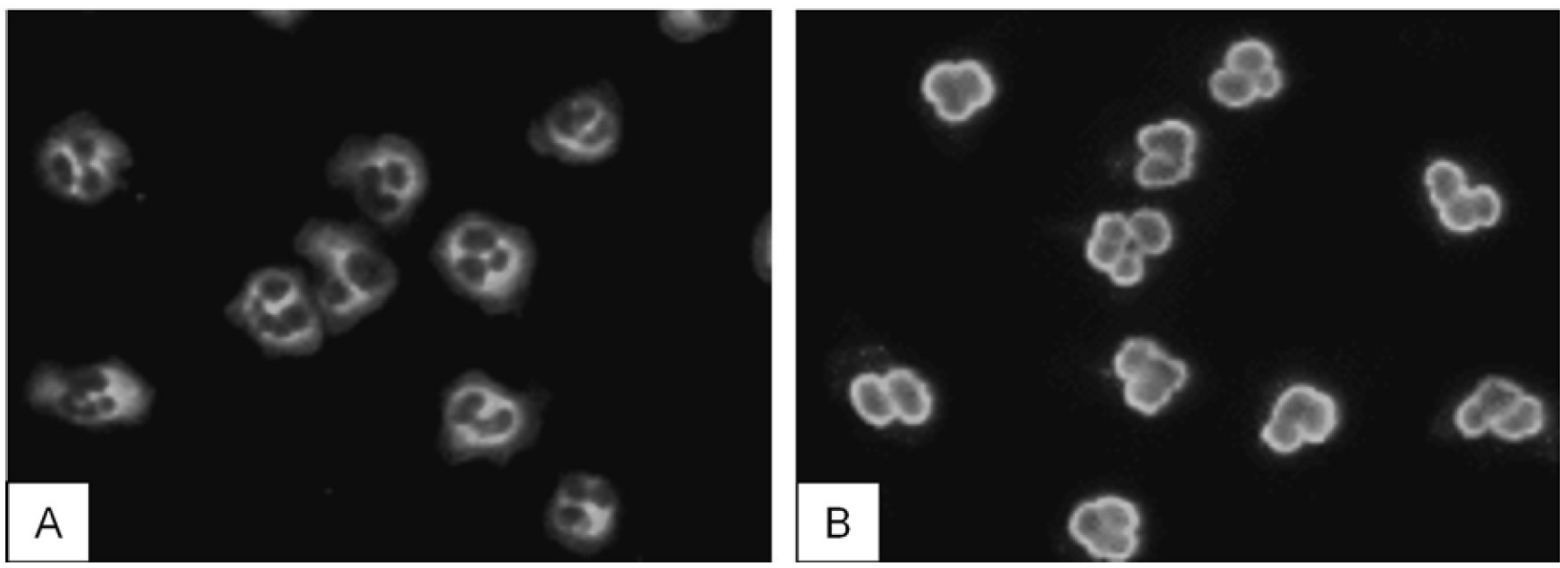

Figure 1-2: The presence of ANCA can be visualized by indirect immunofluorescence on normal human PMNs. (a) Cytoplasmic (C-ANCA) staining observed after incubation with serum positive for PR3-ANCA; (b) Perinuclear (PANCA) pattern observed after incubation with serum positive for MPO-ANCA.

As shown in Figure 1-2, the presence of ANCA in serum can be visualized by indirect immunofluorescence (IIF). In most cases, MPO-ANCA show a perinuclear staining pattern on human PMNs (P-ANCA), whereas PR3-ANCA result in a cytoplasmic staining pattern (CANCA). Testing for ANCA specificities is done by ELISA using purified antigens. For their association with the above-mentioned diseases, determination of ANCA-titers is not only of great value in the diagnosis, but also in the prediction and prevention of relapses during follow-up [7]. Although every organ can be affected by ANCA-associated vasculitis (Table 1-1; reviewed in [5]), severe renal failure and pulmonary hemorrhage in particular result in severe morbidity, and may be fatal if left untreated. Current standard therapy consists of immunosuppression with high dose 
corticosteroids and cyclophosphamide during the first 3-6 months, and azathioprine for 18 months as maintenance therapy [8]. Plasma exchange should be added in case of severe histopathological signs of crescentic glomerulonephritis or fulminant extrarenal disease manifestations. Although this regimen leads to remission in the majority of patients, considerable treatment-related morbidity and failure to induce or maintain remission underline the importance of less toxic and more effective therapies.

Table 1-1: Approximate frequency (\%) of organ-system manifestations in several forms of small-vessel vasculitis (Adapted from Jennette et al., NEJM 1997).

\begin{tabular}{cccccc}
\hline Organ system & $\begin{array}{c}\text { Henoch- } \\
\text { Schönlein } \\
\text { purpura }\end{array}$ & $\begin{array}{c}\text { Cryoglobulinemic } \\
\text { vasculitis }\end{array}$ & $\begin{array}{c}\text { Microscopic } \\
\text { polyangiitis }\end{array}$ & $\begin{array}{c}\text { Wegener's } \\
\text { granulomatosis }\end{array}$ & $\begin{array}{c}\text { Churg- } \\
\text { Strauss } \\
\text { syndrome }\end{array}$ \\
\hline Cutaneous & 90 & 90 & 40 & 40 & 60 \\
Renal & 50 & 55 & 90 & 80 & 45 \\
Pulmonary & $<5$ & $<5$ & 50 & 90 & 70 \\
Ear, nose, and & $<5$ & $<5$ & 35 & 90 & 50 \\
throat & & & & & \\
Musculoskeletal & 75 & 70 & 60 & 60 & 50 \\
Neurological & 10 & 40 & 30 & 50 & 70 \\
Gastrointestinal & 60 & 30 & 50 & 50 & 50 \\
\hline
\end{tabular}

\section{The pathogenesis of ANCA-associated vasculitis - in vitro studies}

The association between small-vessel vasculitides and ANCA has been well established, and a role for the latter in the pathogenesis of disease has been long suggested by multiple in vitro observations. Sera or purified IgG from ANCA-positive patients, as well as monoclonal antibodies directed against MPO or PR3, have been found to induce an oxidative burst in healthy human neutrophils that are pretreated with inflammatory cytokines such as tumor necrosis factor alpha (TNF $\alpha)$ [9-13] and IL-18 [14]. Furthermore, ANCA-activated PMNs are capable of damaging cultured human umbilical vein endothelial cells (HUVECs) [15], and firm adhesion of PMNs to a monolayer of TNF $\alpha$-treated HUVECs is greatly increased after pretreatment with 
ANCA-IgG [16]. Such studies might provide a link between ANCA-positivity and the occurrence of vascular disease. Indeed, freshly isolated and untreated PMNs from patients with ANCAassociated vasculitis are found to produce significantly more superoxide than PMNs from normal controls [17]. From these data, a hypothesis can be deducted, in which three key-players act together to generate full-blown ANCA-mediated disease (Figure 1-3 [18]). The first one is the polymorphonuclear neutrophil (PMN), being the most prominent source of the ANCA antigens, MPO and PR3. The second one, a proinflammatory environment, typically created in vitro by adding a priming concentration of TNF $\alpha$ to the system, is required to "pre-activate" the PMNs, leading to the upregulation of adhesion molecules and, importantly, to an increased availability of the ANCA antigens on the outer membrane of circulating PMNs and monocytes. ANCA then act as the third player by binding to the monocyte and PMN cell membrane, resulting in their activation and the release of lytic granule constituents and reactive oxygen radicals. Finally, activated neutrophils adhere to susceptible endothelium, where they initiate an inflammatory cascade that ultimately results in symptomatology as clinically observed [18].

Although the clinical symptoms of vasculitis patients positive for PR3-ANCA and patients with MPO-ANCA are similar, they differ in a number of ways. This might be consequential to differences in immunogenicity, tissue retention, and binding or functional behavior of the respective autoantigens $[5,19,20]$. MPO is a $140 \mathrm{kD}$ heme protein that is predominantly stored in the lysosomes of monocytes and in the azurophilic granules of resting PMNs. Its capacity to catalyze the formation of hypochlorite $(\mathrm{HOCl})$ out of hydrogen peroxide $\left(\mathrm{H}_{2} \mathrm{O}_{2}\right)$ and chlorideions makes it a powerful tool in the bactericidal armament of these cells. In vitro, MPO however also mediates CD11b/CD18- (MAC-1) mediated binding of PMNs [21] and more importantly, this specific binding of MPO to CD11b/CD18 has recently been shown to result in PMN activation [22]. In addition, there is convincing in vitro and in vivo evidence for a role of MPO in apoptosis [23-25], as well as in direct activation of the complement system [26, 27]. Interestingly, it has been shown that MPO-ANCA are capable of inhibiting the binding of MPO to its natural inhibitor, ceruloplasmin $[28,29]$. The resultant state of uninhibited activity of MPO should not be ignored as a possible pathogenic factor in MPO-ANCA associated vasculitis.

To summarize, during the last decade many factors involved in the pathogenesis of ANCAassociated vasculitis have been elucidated. However, to definitively prove that ANCA are pathogenic, and to further assess their precise role so that new, more targeted and less toxic therapies can be developed, animal models are indispensable. 




Figure 1-3: Representation of ANCA-mediated neutrophil responses that are putatively involved in the pathogenesis of ANCA-associated small vessel vasculitis. (a) Proinflammatory cytokines and chemokines (e.g. tumor necrosis factor $\alpha$ ) released as a result of local or systemic infection cause upregulation of endothelial adhesion molecules (e.g. selectins, ICAM-1, and VCAM) and prime the neutrophil. (b) Neutrophil priming causes upregulation of neutrophil adhesion molecules (CD11b) and translocation of the ANCA antigens from their lysosomal compartments to the cell surface. (c) Engagement of the $\mathrm{F}\left(\mathrm{ab}^{\prime}\right) 2$ portion of ANCA with ANCA antigens on the cell surface and interaction of the Fc part of the antibody with Fc receptors activates the neutrophil, causing increased neutrophil-vessel wall adherence and transmigration. (d) ANCA-mediated neutrophil activation also triggers reactive oxygen radical production and possibly causes neutrophil degranulation, with consequent release of proteolytic enzymes, leading to vasculitis [18] 


\section{The theory of autoantigen complementarity}

Although the role of autoantibodies in a variety of autoimmune diseases is already well established, it is still largely unclear by which mechanism self-tolerance of the human immune system is evaded, and how this leads to the initiation of an autoimmune response. In ANCAassociated vasculitis, a role for molecular mimicry between the autoantigen and an infectious microorganism has been long suspected in this respect (reviewed in [30] and [4]). Indeed, correlations have been found between the occurrence of infection and the development of ANCA-associated vasculitis, but the evidence for molecular mimicry between infectious agents and ANCA antigens being the driving force behind the induction of ANCA has remained weak. With an interesting modification on the theory of idiotypic manipulation (reviewed in [31]), Pendergraft and colleagues recently proposed an alternative mechanism responsible for ANCA induction [32]. In their study, immunization of mice with a peptide translated from the DNA strand complementary to the gene encoding for human PR3 (complementary PR3, cPR3), initially induced an immune response against this peptide (anti-cPR3 IgG). This is followed by an antiidiotypic immune response, that is directed against the antigen-specific part of anti-cPR3 IgG (anti-anti-cPR3 IgG), but also cross-reacts with human PR3, the ANCA autoantigen (Figure 1-4 [32]). Interestingly, the cPR3 peptide bears considerable homology with proteins expressed by a variety of microorganisms, several of which have already been associated with PR3-ANCA associated disease in previous studies. In particular the homology between cPR3 and Staphylococcus aureus proteins is of note, since in several studies, an association is suggested between Staphylococcus aureus positivity and WG (reviewed in [33]).

The most remarkable aspect of the study described above, is the finding that translation of a complementary DNA strand results in a protein that can has the capacity to function as a complementary protein in an idiotype/anti-idiotype network. It remains to be seen whether this is a phenomenon that is more or less unique to PR3, or whether this mechanism is also involved in the generation of other autoantibodies, in particular MPO-ANCA. Nevertheless, although in this non-homologous system the pathogenic role of PR3-ANCA can not be addressed, the theory of autoantigen complementarity provides an attractive explanation for the initiation of the antiPR3 immune response in PR3-ANCA associated vasculitis. 


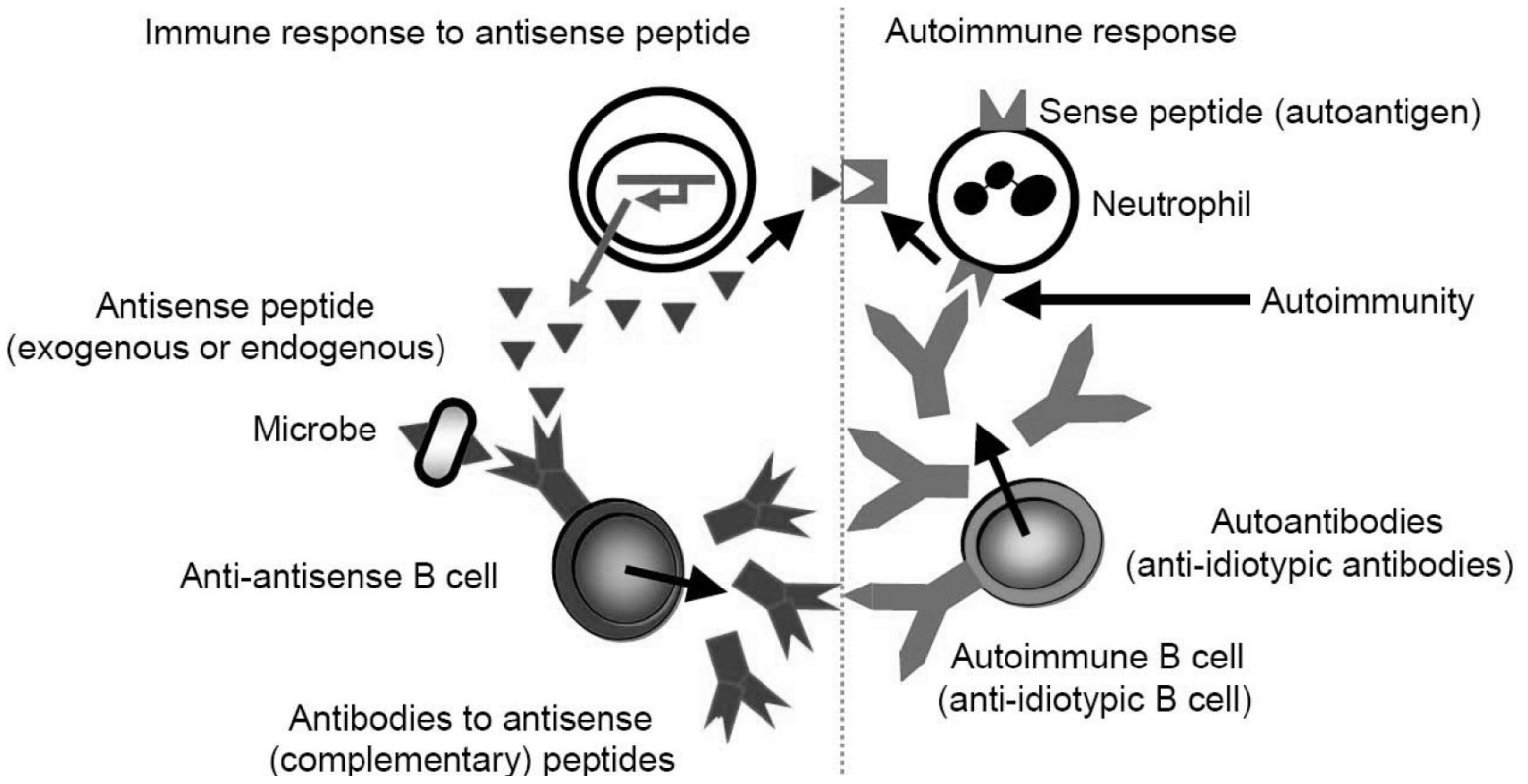

Figure 1-4: Schematic of a new mechanism for the development of autoimmunity, termed the theory of autoantigen complementarity. Autoimmunity is a consequence of an immune response to a protein whose amino acid sequence is complementary to that of a self protein. The immunogen, which elicits the initial immune response (idiotypic response), is complementary in amino acid sequence to the autoantigen. This idiotypic antibody elicits a second immune response (anti-idiotypic response), in which anti-idiotypic antibodies are produced. The anti-idiotypic antibodies are now autoantibodies that react with 'self' [32]

\section{Animal studies of ANCA-associated vasculitis}

\section{A) RATS}

In a rat model of autoimmunity [34], the administration of mercuric chloride $\left(\mathrm{HgCl}_{2}\right)$ to Brown Norway $(\mathrm{BN})$ rats leads to a syndrome characterized by the presence of autoantibodies against a variety of antigens, including DNA, collagen, thyroglobulin, glomerular basement membrane (GBM) components, and MPO [35]. On pathological examination of the animals after sacrifice at days 12-18, moderate acute tubular necrosis and lymphocytic infiltration, in the interstitium as well as perivascularly, can be observed. In addition, in some animals a mild interstitial pneumonitis is seen in the lungs, and multiple other organs contain sites of lymphocytic infiltration. The disease can be divided into an early, T-cell independent, and a late, T-cell dependent phase [36]. The importance of T-cell activation in this model has been confirmed and extended by Macphee et al [37]. They recently showed that blocking CD80 (B7.1) and CD86 (B7.2) on B-lymphocytes inhibits T-lymphocyte activation through inhibition of costimulation via CD28, resulting in decreased caecal vasculitis scores. The above-described model seems well 
applicable to the study of immune responses that are mainly driven by T-cells, such as graftversus-host disease. In contrast, there is still much dispute on the importance of T-lymphocytes in human ANCA-associated vasculitis [38-42], and Xiao et al [43] clearly show the importance of humoral immunity in a study that will be described later in this chapter. To our knowledge, the capability of $\mathrm{HgCl}_{2}$-induced ANCA to induce an oxidative burst in rat PMNs has not been demonstrated thus far, although the observation that administration of antioxidants attenuates $\mathrm{HgCl}_{2}$-induced vasculitis suggests an important role for oxidative stress in this model [44]. The absence of crescentic glomerulonephritis and/or pulmonary granuloma formation, and the multitude of different autoantibodies that are found in this model comprise other important limitations for the use of this model for the study of ANCA-associated vasculitis.

Antibodies against human MPO that cross-react with rat MPO, are observed in BN rats immunized with human MPO $[45,46]$. In such rats, administration of human MPO and $\mathrm{H}_{2} \mathrm{O}_{2}$, the substrate of MPO, via the right jugular vein, leads to pulmonary and gastrointestinal vasculitis, but not glomerulonephritis [46]. In contrast, severe NCGN occurs when kidneys from $\mathrm{BN}$ rats that are immunized with human MPO are perfused with human neutrophil lysosomal extract and $\mathrm{H}_{2} \mathrm{O}_{2}$ [47]. In this study, renal perfusion of MPO-immunized rats with $\mathrm{H}_{2} \mathrm{O}_{2}$ only, or renal perfusion of non-immunized rats, did not lead to glomerulonephritis, whereas perfusion of immunized rats with MPO led to moderate immune complex glomerulonephritis. Interestingly, on pathological evaluation deposits of $\operatorname{Ig} G$ are found in kidneys of rats sacrificed 4 or 24 hours after perfusion, but are absent at later timepoints. This suggests that in fact immune complexes could play a role in the pathogenesis of NCGN, but that they might already have disappeared by the time kidney biopsies are generally taken. However, in contrast to these results considerable immune depositions were found by Yang et al. in BN rats as well as in spontaneously hypertensive rats at day 10 after perfusion [48].

Recently, a novel rat model of ANCA-associated vasculitis is presented that provides strong evidence for pathogenicity of the anti-MPO immune response in this disease [49]. In this model, immunization of Wistar Kyoto (WKY) rats with human MPO leads to the generation of antibodies against human MPO, that cross-react with rat neutrophils. In addition, on pathologic examination after 8 weeks, MPO-immunized rats display mild pauci-immune crescentic glomerulonephritis that is histopathologically similar to human ANCA-associated glomerulonephritis. In the lungs, signs of alveolar hemorrhage, perivascular cuffing by leukocytes and fibrin deposits are also found. The close resemblance between the histopathology seen in this 
model and in humans, makes this a promising model for the study of ANCA-associated vasculitis and its pathogenesis.

Also in Wistar rats, the role of bacterial infection in ANCA-associated vasculitis (reviewed in [4] and [30]) was recently investigated [50]. Immunization with pasteurized and sonicated preparations of S. aureus or E. coli led to segmental glomerular sclerosis, albeit in a very limited number of animals. Rats were given injections of the preparation together with Complete or Incomplete Freund's Adjuvant at 10 day intervals and sacrificed at day 60 or 70 . The affected E. coli-immunized rat was found to be C-ANCA positive, but antigen specificity could not be determined, nor could T-cell reactivity be observed in response to MPO or PR3.

\section{B) MICE}

In 1995, Blank et al. reported the development of a novel animal model in which PR3-ANCAassociated vasculitis can be studied [51]. Based on the theory of idiotypic manipulation, mice were immunized with human anti-PR3, resulting in the development of murine antibodies to human PR3 at 4 months. Anti-PR3 IgG-immunized, but not normal human IgG-immunized mice sacrificed at 8 months showed massive sterile micro-abscesses or perivascular lymphocytic infiltration, but not necrotizing vasculitis, in the lungs. Remarkably, immunization with human PR3-ANCA also led to elevated titers of mouse antibodies against human MPO and endothelial cells. Reactivity of murine sera to murine antigens was not examined. In contrast, Jenne et al. found that autoantibodies from 40 different WG patients do not bind to the murine homologue of PR3 [52]. It is therefore unlikely that the histopathology observed by Blank et al. can be attributed to the presence of mouse anti-human PR3.

Rauova et al [53] reported the development of antibodies to human MPO in wild type (WT) mice after immunization with live or apoptotic human PMNs. Incubation of unprimed human PMNs with these antibodies led to an increased respiratory burst compared to control mouse IgG. It is however unclear whether these antibodies bind to and activate murine PMNs, and histological examination after sacrifice at several timepoints revealed no pathologic alterations. Nevertheless, this study underlines the potential role of apoptosis in the initiation of ANCA production, a notion that was confirmed in other animal studies (e.g. ref [54]; discussed in more detail in Chapter 7). In this respect, a study by Clayton et al. showed that apoptotic neutrophils are phagocytosed by dendritic cells (DCs), an effect that was enhanced by incubation with ANCA [55]. This however did not lead to sensitization of DCs, but to reduced allogeneic $\mathrm{T}$ cell responses, an effect that was abolished by coincubation with TNF $\alpha$. 
SCG $/ \mathrm{Kj}$ mice are the product of breeding with (BXSB x MRL/Mp-lpr/lpr) F1 mice that are selected for having parents with the most severe crescentic glomerulonephritis (CGN) [56]. BXSB is a mouse strain that spontaneously develops autoimmune disease and immune complex glomerulonephritis [57], and MRL/Mp-lpr/lpr mice spontaneously develop neutrophilic vasculitis with histological features of microscopic angiitis $[58,59]$. Interestingly, in the latter strain some mice also develop anti-MPO autoantibodies, and their presence is strongly associated with the occurrence of clinical vasculitis. SCG/Kj mice however spontaneously develop CGN and proteinuria to a higher degree than MRL/Mp-lpr/lpr or BXSB mice [56]. Systemic vasculitis is also observed, although the kidneys are not frequently affected. Immunohistochemically, deposits of $\operatorname{IgG}$, IgM and C3 in the glomeruli are scanty, and serum levels of immune complexes and anti-DNA antibodies were equal to, or lower than the levels observed in MRL/Mp-lpr/lpr mice. However, Neumann et al. recently reported significant glomerular immune deposits in all animals with CGN, and found no evidence for ANCA [60]. The contrast with small-vessel vasculitis in humans, a disease predominantly characterized by paucity of immune reactants, is evident, and makes the SCG/Kj model not representative for human ANCA-associated vasculitis.

\section{The mouse model of anti-MPO IgG-induced glomerulonephritis}

Recently, Xiao et al. provided strong evidence that MPO-ANCA play a pivotal role in the development of MPO-ANCA-associated vasculitis [43]. They described an animal model in which an immune response against murine MPO is raised in mice lacking MPO [61], by immunization with murine MPO (muMPO) isolated from WEHI-3, a murine myeloid cell line. Adoptive transfer of splenocytes from muMPO-immunized $M p o^{-/}$mice to RAG2 knockout $\left(\operatorname{Rag}^{-/}\right)$mice, that lack mature B and T lymphocytes, induces a dose-dependent elevation of antimuMPO titers, that kept rising until sacrifice at day 13. In mice receiving $\geq 5 \times 10^{7}$ anti-muMPO splenocytes, markedly elevated BUN and serum creatinine levels, as well as hematuria, proteinuria and leukocyturia were seen. Moreover, on pathologic evaluation, extensive focal necrotizing glomerulonephritis could be observed, with crescent formation and/or necrosis in $80 \%$ of glomeruli. Also in other organs such as the lungs, spleen, and lymph nodes, manifestations of vascular inflammation were found. Transfer of splenocytes from mice immunized with bovine serum albumin (BSA) to Rag2 ${ }^{-1}$ mice, as well as the transfer of muMPO-immunized splenocytes to $M p o^{-1-}$ mice, only led to a mild immune complex glomerulonephritis. 




Figure 1-5: Transfer of IgG from $M p o^{-/}$mice immunized with murine MPO to wildtype or immune-deficient $\left(\operatorname{Rag}^{-/}\right)$ mice results in NCGN, whereas splenocytes or IgG from sham (BSA-) immunized $M p 0^{-/}$mice do not [43]

In addition to the above-described adoptive transfer experiments, the effect of a passive transfer of $\mathrm{IgG}$ from muMPO-immunized $\mathrm{Mpo}^{-/-}$mice to WT or Rag $2^{-/}$mice was investigated. Sera from immunized mice were pooled and $\operatorname{IgG}$ was isolated and injected intravenously into WT and Rag2 ${ }^{-1}$ mice (see Figure 1-5 for a schematic representation of the mouse model). After three days, both groups had comparable anti-MPO titers. Both groups also developed urine abnormalities, although $\mathrm{Rag}^{-1}$ mice tended to be somewhat more affected than WT mice. On pathologic evaluation at 6 days, WT as well as Rag ${ }^{-1}$ mice had mild focal necrotizing glomerulonephritis with crescents. Notably, the percentages of crescentic and necrotic glomeruli were considerably higher in Rag2 ${ }^{\%}$ than in WT mice. Control mice from both groups that received $\operatorname{IgG}$ isolated from BSA-immunized $\mathrm{Mpo}^{-/-}$mice, developed no urine or pathologic abnormalities. Remarkably, when compared to the adoptive transfer of anti-muMPO splenocytes, passive transfer led to less 
severe urine abnormalities and lower levels of necrosis and crescent formation. In addition, BUN and serum creatinine levels were not increased in the passive transfer experiments.

The mouse model described above elegantly proves that MPO-ANCA play an important role in the pathogenesis of ANCA-associated vasculitis and NCGN. However, it is remarkable that, although ANCA titers after passive transfer and adoptive transfer are comparable, disease manifestations are much more severe after adoptive transfer. This might be an effect of the described presence of low to moderate amounts of immune complex depositions in the kidneys of Rag $2^{-}$mice receiving splenocytes [62]. Moreover, it suggests considerable contributions of other factors that are introduced by the transfer of splenocytes. There is support from human studies for a role of autoreactive T-cells in the development of vasculitis [39-42]. T-cell proliferation in response to MPO is also increased in rats immunized with human MPO, as determined by delayed-type hypersensitivity (DTH) reaction and T-cell proliferation assay [47, 50]. Therefore, it might be interesting to identify muMPO-reactive T-lymphocytes in the spleens of muMPO-immunized $\mathrm{Mpo}^{-/}$mice, and to investigate whether adoptive transfer of these cells leads to disease. In addition, to extend the in vitro observed effect of neutrophil priming on ANCA-induced neutrophil oxidative burst [9-13], the animal model provides the opportunity to study the effect of pro-inflammatory stimuli on MPO-ANCA induced glomerulonephritis.

It might seem highly artificial to induce disease through the transfer of murine MPO-specific $\operatorname{IgG}$. It is therefore interesting to note in this respect a recently published case report, in which the newborn from an MPO-ANCA positive mother develops severe pulmonary hemorrhage, associated with MPO-ANCA positivity [63]. Upon the initiation of therapy with corticosteroids and exchange transfusion, the symptoms subsided, and the neonate has remained in remission during one year of follow-up. Although only casuistic, this report is the first to convincingly show pathogenicity of MPO-ANCA alone, and provides strong support for the clinical relevance of the observations in the murine transfer model as discussed above.

The success of the transfer models of vasculitis mediated by MPO-ANCA raised the question whether it was possible to establish a similar mouse model of PR3-ANCA associated disease. To address this question, Pfister and colleagues immunized mice double-deficient for PR3 and neutrophil elastase (NE) with recombinant murine PR3, and transferred their sera to WT mice [64]. The administration of serum from mPR3-immunized PR3-/NE-double-deficient mice was shown to significantly augment TNF $\alpha$-induced local panniculitis in WT mice. Remarkably however, neither healthy WT mice, nor WT mice pretreated with bacterial lipopolysaccharide (LPS) as a proinflammatory stimulus, developed vasculitis or glomerulonephritis upon serum 
transfer. Whether the apparent discrepancy between the murine model of anti-MPO IgG-induced NCGN and its PR3 equivalent is consequential to differences in the administered titer, or caused by biochemical or biophysical differences between murine MPO and murine PR3 is as yet unexplained. It can however be concluded that a suitable animal model of this disease should be awaited before a valid conclusion can be drawn regarding the pathogenicity of PR3-ANCA in clinical disease.

\section{Conclusion}

During the past two decades, a number of animal models have been used to study ANCAassociated vasculitis. Based on in vitro observations, it was suggested that ANCA play an important role in the pathogenesis of this disease, although direct evidence for a causal relation between ANCA and disease was absent. The transfer studies performed by Xiao et al., in which the administration of $\operatorname{IgG}$ from muMPO-immunized $\mathrm{Mpo}^{-/-}$mice to MPO-positive mice causes a disease remarkably similar to human NCGN and vasculitis [43], provide an animal model that is excellently suitable to investigate the link between the anti-MPO immune response and the occurrence of disease.

To summarize, recently developed animal models of ANCA-associated vasculitis provide promising tools to study the pathogenesis of this disease. As a result, they might be beneficial to the development of novel therapies. As long as a validated animal model of PR3-ANCAassociated vasculitis is absent, progress in this regard needs to be awaited.

\section{Outline of this thesis}

The main goal of this thesis is to create insight in the various factors involved in the initial phase of the development of ANCA-associated vasculitis. To this end, we utilized the mouse model of anti-MPO IgG-induced glomerulonephritis as described above, and as schematically represented in Figure 1-5. In Chapter 2, a study is presented in which we test the hypothesis obtained from in vitro studies, that proinflammatory stimuli enhance the pathogenic effect of anti-MPO antibodies. We show that LPS increases the severity of anti-MPO IgG-induced NCGN. This resulted in a modified model that better expresses the severity of human MPO-ANCA associated disease. In Chapter 3, the rationale for TNF $\alpha$ bioactivity-inhibiting therapy in humans with ANCAassociated vasculitis is discussed, bearing in mind in vitro observations, the results of various animal studies, and mounting data from clinical studies on the efficacy of various TNF $\alpha$ inhibiting drugs. 
A recent study, performed in various complement-deficient mouse strains, shows a crucial role for the complement system in anti-MPO IgG-induced NCGN. Chapter 4 describes a study in which the mouse model of anti-MPO IgG-induced NCGN is used to investigate whether complement factor $\mathrm{C} 5$ could be a therapeutic target in ANCA-associated vasculitis.

In the mouse model of anti-MPO IgG-induced NCGN, disease is induced with polyclonal antiMPO IgG, isolated from the sera of $M p o^{-/}$mice immunized with murine MPO. In Chapter 5, we describe the generation and characterization of a panel of monoclonal antibodies (moAbs) specific for murine MPO, and test their pathogenicity in the context of a systemic proinflammatory stimulus induced by the administration of LPS, as well as in the context of a local proinflammatory environment created by the administration of a low dose of heterologous antibodies specific for the glomerular basement membrane (GBM).

As discussed above, the symptomatology of MPO-ANCA associated vasculitis might be explained in part by the biological function of MPO itself. Consequently, a better understanding of the function of MPO in inflammation might provide additional insight into the pathogenesis of MPO-ANCA associated glomerulonephritis as well. Since it is impossible to study MPOANCA associated NCGN in $M{ }^{-1-}$ mice, we employed a murine model of renal warm ischemia/reperfusion injury to provide more insight in the role of MPO in renal inflammation. The results of this study are presented in Chapter 6. Finally, in Chapter $\mathbf{7}$ the results of ongoing investigations, obtained by our group as well as others, in the field of ANCA-associated vasculitis are summarized, discussed, and implemented in a modified theory, covering the pathophysiological mechanisms involved in the initial development of disease as well as the mechanisms that might be responsible for its perpetuation. 

Chapter 2 - Aggravation of anti-myeloperoxidase antibody-induced glomerulonephritis by bacterial lipopolysaccharide: role of tumor necrosis factor $\alpha$

Huugen D, Xiao H, van Esch A, Falk RJ, Peutz-Kootstra CJ, Buurman WA, Tervaert JW, Jennette JC, Heeringa P. Am J Pathol. 2005 Jul;167(1):47-58. 


\section{Abstract}

Wegener's granulomatosis, microscopic polyangiitis, Churg-Strauss syndrome, and idiopathic pauci-immune necrotizing crescentic glomerulonephritis (NCGN) are associated with anti-neutrophil cytoplasmic autoantibodies (ANCA). There is clinical and experimental evidence indicating that ANCA and pro-inflammatory stimuli of infectious origin act synergistically in causing vasculitis. Here, we tested this hypothesis using a recently developed mouse model of anti-MPO IgG induced glomerulonephritis and bacterial lipopolysaccharide (LPS), as a model proinflammatory stimulus.

Systemic administration of LPS dose-dependently increased renal injury induced by anti-MPO IgG as demonstrated by increased glomerular crescent formation and glomerular necrosis. In the early phase, LPS enhanced anti-MPO IgG induced glomerular neutrophil accumulation. Furthermore, a transient induction of circulating levels of TNF $\alpha$, followed by a marked increase in circulating levels of myeloperoxidase was observed upon administration of LPS. In vitro, anti-MPO IgG induced a respiratory burst in murine neutrophils only after priming with TNF $\alpha$. Finally, antiTNF $\alpha$ treatment attenuated, but did not prevent, the LPS mediated aggravation of anti-MPO IgG induced glomerulonephritis.

In conclusion, our study demonstrates that ANCA and pro-inflammatory stimuli act synergistically in inducing vasculitic disease and point to a potentially beneficial effect of TNF $\alpha$-bioactivity inhibiting treatment modalities on human ANCA-associated NCGN. 


\section{Introduction}

Wegener's granulomatosis (WG), microscopic polyangiitis (MPA), Churg-Strauss syndrome and idiopathic pauci-immune necrotizing crescentic glomerulonephritis (NCGN) are forms of smallvessel vasculitis of unknown etiology that are strongly associated with anti-neutrophil cytoplasmic autoantibodies (ANCA) [3]. ANCA comprise a group of autoantibodies directed against proteins contained in the lysosomal compartments of neutrophils and monocytes. The primary target antigens have been identified as proteinase $3(\operatorname{Pr} 3)$, a $29 \mathrm{kD}$ neutral serine proteinase, and myeloperoxidase (MPO), a $140 \mathrm{kD}$ protein involved in the generation of reactive oxygen species [65]. To date, detection of ANCA has proven to be a helpful diagnostic tool and many clinical studies have confirmed that Pr3-ANCA and MPO-ANCA are highly specific for WG and MPA, respectively [65]

Since the discovery of ANCA, numerous clinical and laboratory studies have been performed to determine whether ANCA are directly involved in the pathogenesis of vasculitis and glomerulonephritis. The overall concept derived from these studies is that ANCA induced vasculitis is a 'two-hit' process in which ANCA together with pro-inflammatory stimuli, most likely of infectious origin, are required for the induction of full-blown disease. In support of this model are clinical observations demonstrating that relapses in disease activity are often preceded by rising ANCA levels [66-68]. Furthermore, the frequent observation of infectious episodes prior to diagnosis and/or relapse suggests that these may play a role in the pathogenesis of ANCA-associated disease [69-72]. Consistent with these clinical observations are in vitro studies that have shown that ANCA are capable of activating neutrophils and monocytes primed with pro-inflammatory cytokines, resulting in an oxidative burst, degranulation, production of cytokines and endothelial cell damage $[9-13,73]$.

Recently, an experimental animal model of anti-MPO induced NCGN was developed that involves the adoptive transfer of mouse MPO-reactive splenocytes into immune-deficient mice [43]. These mice developed severe NCGN with pathological features that were remarkably similar to human anti-MPO associated glomerulonephritis. In addition, it was demonstrated that passive transfer of murine anti-mouse MPO antibodies alone into either immune-deficient or wildtype mice also induces NCGN, although of a substantially milder form.

Thus, the association between ANCA, small vessel vasculitis, and infections suggests that, besides ANCA, a second (nonspecific) pro-inflammatory signal is necessary to induce full-blown disease. To test this hypothesis, we used the experimental mouse model of anti-MPO antibody induced 
NCGN and investigated the effects of bacterial lipopolysaccharide (LPS), as a model (pro-) inflammatory stimulus, on disease severity.

\section{Material and methods}

\section{MICE}

$\mathrm{MPO}^{-/}$mice, backcrossed to a C57Bl/6 background for 6 times, were genotyped using PCRamplified DNA from tail clippings [61]. Wildtype (WT) female C57Bl/6 (8-12 weeks of age) were obtained from Harlan (Horst, the Netherlands). Mice were kept according to University of Maastricht animal facility regulations, and all experiments were approved by the local Animal Care and Experimentation Committee.

\section{PURIFICATION OF MURINE MPO}

Murine MPO (muMPO) was purified from WEHI-3, a myeloid cell line, that was grown in HEPES-buffered McCoy5A medium containing penicillin/streptomycin and 10\% FCS. When a density of $1.5 \times 10^{6}$ cells $/ \mathrm{ml}$ was reached, cells were harvested, resuspended in buffer A containing 6.7 $\mathrm{mM}$ sodium acetate, $3.0 \mathrm{mM} \mathrm{MgCl}, 3.0 \mathrm{mM} \mathrm{NaCl}, 0.5 \mathrm{mM}$ PMSF and 1\% CTAB, and lysed by Dounce homogenization on ice. After stirring $\left(2\right.$ hours, $\left.4^{\circ} \mathrm{C}\right)$, insoluble particles were removed by centrifugation $\left(14.000 \mathrm{~g}, 30\right.$ minutes, $\left.4^{\circ} \mathrm{C}\right)$, and the supernatant was dialyzed overnight at $4^{\circ} \mathrm{C}$ against buffer $\mathrm{B}$ containing $100 \mathrm{mM}$ sodium acetate $\left(\mathrm{pH}\right.$ 6.3) and $100 \mathrm{mM} \mathrm{NaCl}$. Next, $\mathrm{CaCl}_{2}$, $\mathrm{MgCl}_{2}$ and $\mathrm{MnCl}_{2}$ were added to a final concentration of $1 \mathrm{mM}$ each, and the solution was mixed with concanavalin A-Sepharose (Amersham Biosciences, Roosendaal, Netherlands). After centrifugation and removal of supernatant, the con A was resuspended in several washes of buffer B with $750 \mathrm{mM}$ methyl a-D-mannopyranoside to elute the MPO (overnight, $4^{\circ} \mathrm{C}$ ). The samples of buffer B containing MPO (as judged by OD at $428 \mathrm{~nm}$ ) were then dialyzed against buffer C, containing $50 \mathrm{mM}$ sodium acetate ( $\mathrm{pH} 8.5-9)$ and $100 \mathrm{mM} \mathrm{NaCl}$, loaded onto a Mono S cation exchange column (Biorad, Veenendaal, the Netherlands), eluted with $1 \mathrm{M} \mathrm{NaCl}$, and dialyzed against PBS. Purity was checked by SDS-PAGE.

\section{PREPARATION OF PATHOgENIC MOUSE ANTI-MURINE MPO IGG AND CONTROL MOUSE ANTI-BSA IGG.}

$\mathrm{MPO}^{-/}$mice were immunized as reported previously[43] with minor modifications. Briefly, mice received an intraperitoneal injection of $10 \mu \mathrm{g}$ of muMPO in complete Freund's adjuvant (Difco) 
on day 0 , followed by intraperitoneal booster injections in incomplete Freund's adjuvant on days 21 and 36. On day 42, blood was obtained and mice were sacrificed. Antibody titers were monitored by enzyme-linked immunosorbent assay (ELISA) as described previously [43]. In short, microtiter plates were coated overnight with muMPO $(0.5 \mu \mathrm{g} / \mathrm{ml})$ and blocked with bovine serum albumin (BSA). Then plates were incubated with mouse sera (1:100 starting dilution), followed by incubation with alkaline phosphatase-conjugated goat-anti-mouse $\operatorname{Ig} G$ antibodies. 4nitrophenyl phosphate (pNPP) was used as substrate, and wells were analyzed spectrophotometrically at $405 \mathrm{~nm}$.

To obtain anti-BSA $\operatorname{IgG}$, a separate group of $\mathrm{MPO}^{-/}$mice was immunized with BSA following the protocol described above. Normal serum from C57Bl/6 mice was obtained from Harlan. IgG was isolated from pooled sera by $50 \%$ ammonium sulphate precipitation followed by protein $\mathrm{G}$ column affinity chromatography. IgG containing fractions were concentrated by ultrafiltration (Centriplus, Millipore, Amsterdam, the Netherlands) and dialyzed against PBS. Protein concentrations were determined using the bicinchoninic acid protein assay kit (Pierce, Rockford, IL). The anti-MPO titer of each batch was checked by ELISA as described above, using a sample of anti-MPO $\operatorname{IgG}$ with previously established pathogenicity as a reference. Endotoxin concentrations were determined by the limulus amebocyte lysate assay (Bio Whittaker, Walkersville, MD) and depending on the isolation ranged from $0.04 \mathrm{ng} / \mathrm{ml}$ to $1.2 \mathrm{ng} / \mathrm{ml}$.

\section{INDUCTION OF GLOMERULONEPHRITIS BY PASSIVE TRANSFER OF ANTI-MPO IGG}

C57Bl/ 6 mice received a dose of $100 \mu \mathrm{g} / \mathrm{g}$ body weight of sterile-filtered $(0.2 \mu \mathrm{m}$ filter, Schleicher\&Schuell, Dassel, Germany) anti-MPO IgG by intraperitoneal injection. Where stated, groups of mice additionally received a single intraperitoneal injection of 5.0, 0.5 , or $0.05 \mu \mathrm{g} / \mathrm{g}$ of LPS (E. coli, serotype 026-B6, Sigma St. Louis MO, $n=4-5$ in each group) dissolved in sterile PBS one hour after the administration of IgG. Control mice were injected with anti-BSA IgG (100 $\mu \mathrm{g} / \mathrm{g}, \mathrm{n}=5)$ followed by $5.0 \mu \mathrm{g} / \mathrm{g}$ of LPS one hour later, or with LPS (5.0 $\mu \mathrm{g} / \mathrm{g}, \mathrm{n}=4)$ alone. Circulating anti-MPO IgG was monitored by ELISA as described above, using a serum pool from MPO-immunized $\mathrm{MPO}^{-/}$mice as reference. Results are expressed as a percentage of the absorbance units (OD $405 \mathrm{~nm}$ ) of a positive control pool serum.

\section{MEASUREMENT OF SERUM MPO AND TNF $\alpha$ LEVELS}

In a separate experiment, the effect of intraperitoneal injection of LPS on serum TNF $\alpha$ - and MPO levels was determined. Blood was taken from mice 1 week before, 1 hour after and 1 and 6 
days after the administration of $0.05,0.5$ or $5.0 \mu \mathrm{g} / \mathrm{g}$ LPS ( $\mathrm{n}=4$ in each group) in endotoxin-free $0.9 \%$ saline. Samples were centrifuged and serum was taken and stored at $-20^{\circ} \mathrm{C}$.

To detect circulating MPO, we generated a mouse-anti-mouse MPO monoclonal antibody (moab) from splenocytes obtained from muMPO-immunized $\mathrm{MPO}^{-/}$mice using standard procedures. The resulting moab (IgG1, designated 8F4) recognized murine MPO and crossreacted with rat but not human MPO as determined by direct ELISA and immunohistochemistry on normal spleen sections (data not shown). Using this antibody, a catching ELISA was developed for the detection of murine MPO in sera as follows. Microtiter plates were coated with Fc $\gamma$ fragment-specific goat anti mouse-IgG $(3.2 \mu \mathrm{g} / \mathrm{ml}, 100 \mu \mathrm{l} /$ well; Jackson ImmunoResearch), incubated for 48 hours at $4^{\circ} \mathrm{C}$, and blocked with $1 \%$ BSA in PBS for 30 minutes at room temperature. Plates were then incubated with $8 \mathrm{~F} 4(1.0 \mu \mathrm{g} / \mathrm{ml}, 100 \mu \mathrm{l} /$ well $)$ for 1 hour at room temperature. Next, plates were incubated with appropriately diluted serum samples for 1 hour at room temperature, followed by incubation with polyclonal rabbit anti-human MPO $(14 \mu \mathrm{g} / \mathrm{ml}$, DakoCytomation, Glostrup, Denmark) for 1 hour at room temperature and incubation with alkaline phosphatase-labeled polyclonal goat anti-rabbit IgG for 1 hour at room temperature. Finally, plates were incubated with pNPP for 30 minutes, and results were analyzed spectrophotometrically at $405 \mathrm{~nm}$. Concentrations were calculated from a standard curve of purified murine MPO (range 2.5-100 ng/ml).

TNF $\alpha$ levels were determined by capture ELISA as described previously [74]. Briefly, plates were coated overnight with TN3 $(5 \mu \mathrm{g} / \mathrm{ml})$. After blocking with BSA, samples were added to the plate for 1 hour at room temperature. Plates were then incubated for $1 \mathrm{~h}$ with rabbit anti-mouse TNF polyclonal antibody followed by incubation with peroxidase-labeled goat anti-rabbit $\operatorname{IgG}$ for 1 hour. The assay was developed with O-Phenylene Diamine (OPD), the reaction was stopped with 4N H2SO4, and the OD was measured at $490 \mathrm{~nm}$. Concentrations were calculated from a standard curve of recombinant murine TNF $\alpha$ (range $0.02-40 \mathrm{ng} / \mathrm{ml}$ ).

\section{ANTI-TNF $\alpha$ TREATMENT}

To investigate the effects of TNF $\alpha$ depletion on disease induction, groups of mice received a single intraperitoneal dose of the anti-murine TNF $\alpha$ antibody TN3 (500 $\mu \mathrm{g} /$ mouse, endotoxin concentration $<10 \mathrm{pg} / \mathrm{ml}$ ), a complementarity-determining regions-grafted murine IgG2a (kindly provided by Celltech, Slough, UK, $\mathrm{n}=8$ ) or isotype control (moab L2-3D9, endotoxin concentration $<10 \mathrm{pg} / \mathrm{ml}, \mathrm{n}=7$ ), $[75,76]$ in sterile PBS, 24 hours before anti-MPO IgG and LPS 
$(0.5 \mu \mathrm{g} / \mathrm{g})$ administration. When given 24 hours in advance, this dose of TN3 completely inhibited TNF $\alpha$ activity in sera of mice taken one hour after intraperitoneal injection of $0.5 \mu \mathrm{g} / \mathrm{g}$ LPS, as determined by cytotoxicity assay using the murine fibrosarcoma cell line WEHI 164 as described previously [77]. All mice were sacrificed six days after disease induction.

\section{LABORATORY AND PATHOLOGICAL EVALUATION OF DISEASE INDUCTION}

At the times indicated, mice were bled and sacrificed. Urine samples were tested by dipstick (Bayer, Mijdrecht, the Netherlands) for hematuria, proteinuria, and leukocyturia and scored on 0$4+$ scale. Blood urea nitrogen (BUN) and creatinine levels were determined in sera collected at the time of sacrifice by an enzymatic degradation assay on a Synchron LX20 PRO (Beckman Coulter Inc., Fullerton CA).

Tissue samples were taken from both kidneys and processed for light microscopy, immunofluorescence, and immunohistochemistry. For light microscopy, renal tissue samples were fixed in $4 \%$ formaldehyde and embedded in paraffin. $1.5 \mu \mathrm{m}$ sections were cut and hematoxylin/eosin and periodic acid Schiff staining were performed. For each animal, a crescent score was determined by evaluating crescent formation in 50 consecutive glomerular cross sections. Only glomeruli that had two or more layers of cells in Bowman's space were considered crescentic. Similarly, a glomerular necrosis score was determined for each animal by evaluating segmental or global glomerular capillary necrosis in 50 consecutive glomerular cross sections. Analysis was performed in a blinded fashion using coded slides.

Phenotypic analysis of the inflammatory cell infiltrate was performed on $4 \mu \mathrm{m}$ cryostat sections fixed in $100 \%$ acetone at room temperature. The following primary antibodies were used: rat anti-mouse CD45, rat anti-mouse neutrophils (clone NIMP-R14)[78]; rat anti-mouse CD68 (macrophages, clone FA11)[79]; rat anti-mouse CD3 (clone KT3). Endogenous peroxidase activity was blocked with $0.05 \% \mathrm{H}_{2} \mathrm{O}_{2}$ in PBS. Rabbit anti-rat IgG-PO and goat anti-rabbit IgGPO (both DakoCytomation) were used as secondary and tertiary antibodies, respectively. Antibody binding was visualized using 3-amino-9-ethylcarbazole (AEC) and $\mathrm{H}_{2} \mathrm{O}_{2}$ as substrates. Sections were counterstained with hematoxylin. Glomerular cell infiltrates were determined by counting the number of positive cells in 30 glomerular cross sections per kidney section. In experiments where effects of LPS on early (day 1) neutrophil recruitment were studied the number of glomeruli containing neutrophil aggregates was also scored. An aggregate was defined as a homotypic aggregate of 3 or more neutrophils and was evaluated in 30 glomerular cross sections. 
Mouse $\operatorname{IgG}$ and fibrin deposits were detected by immunofluorescence using rabbit anti-mouse IgG-Alexa Fluor 488 (Molecular probes, Leiden, the Netherlands) and rabbit anti-human fibrinogen-FITC (DakoCytomation). MPO deposits were detected using biotinylated mouse antimouse MPO (8F4) followed by streptavidin-Alexa 488 (Molecular probes). Endogenous avidin and biotin were blocked using a streptavidin-/biotin blocking kit (Vector Laboratories, Burlingame, CA).

\section{SUPEROXIDE ANION ASSAY}

To determine the in vitro capacity of polyclonal mouse-anti murine MPO antibodies to induce an oxidative burst in murine neutrophils, we used the superoxide dismutase inhibitable (SOD) ferricytochrome C assay [80]. Peritoneal exudate cells (PECs) were obtained by flushing of the peritoneal cavities of 3 to $4 \mathrm{WT}$ or $\mathrm{MPO}^{-/-}$mice with sterile Hank's balanced salt solution (HBSS) four hours after intraperitoneal administration of $1 \mathrm{ml} \mathrm{3 \%}$ thioglycollate. As determined on May Grünwald Giemsa-stained cytospots, the percentage of neutrophils in these cell preparations was $\geq 55 \%$. Cells were centrifuged and resuspended at a concentration of $2 \times 106$ cells $/ \mathrm{ml}$ in HBSS. The remainder of the procedure was performed at $37^{\circ} \mathrm{C}$. Cells were incubated with cytochalasin B (5 $\mu \mathrm{g} / \mathrm{ml}$, Serva, Heidelberg, Germany) for 5 minutes, followed by incubation with recombinant murine TNF $\alpha(10 \mathrm{ng} / \mathrm{ml}, \mathrm{R} \& \mathrm{D}$, Abingdon, UK) or an equal volume of HBSS for 10 minutes. Next, 96-well microtiter plates were incubated with $200 \mu$ l cell suspension $(4 \times 105$ cells/well), ferricytochrome C (70 $\mu \mathrm{M}$, Sigma, Zwijndrecht, the Netherlands), stimulus, and either SOD (42 U/ml, Sigma) or an equal volume of HBSS. As stimulus, $250 \mu \mathrm{g} / \mathrm{ml}$ anti-muMPO IgG or normal mouse IgG was used. Phorbol ester myristate acetate (PMA, 200 ng/ml, Sigma) served as a positive control. Optical density at $550 \mathrm{~nm}$ was measured every 15 minutes for 60 minutes and SOD-inhibitable production of superoxide was determined as $\Delta \mathrm{OD}$ at $550 \mathrm{~nm}$. Each test was performed in triplicate and experiments were repeated $3\left(\mathrm{MPO}^{-/}\right.$cells) to 4 (WT cells) times.

\section{STATISTICAL ANALYSIS}

Data are expressed as means $\pm \mathrm{SD}$ and were analyzed using the unpaired two-tailed Student's $\mathrm{t}$ test using Graphpad Prism 4.01 for Windows (Graphpad Software, San Diego CA, USA). For semiquantitative data from dipstick analysis, a ranked ANOVA test was used to evaluate differences across all groups. When significant differences were detected with ANOVA, the Duncan test was used to evaluate statistical differences between specific groups. A p-value $\leq 0.05$ was considered statistically significant. 


\section{Results}

\section{DEVELOPMENT OF CIRCULATING MPO-ANCA AND URINARY ABNORMALITIES AFTER PASSIVE TRANSFER OF ANTI-MPO IGG}

Upon passive transfer of anti-MPO IgG, circulating anti-MPO antibodies were detected in all subjected mice. The level of circulating anti-MPO was highest on day 1 and had declined at the time of sacrifice (day 6). On day 1, no differences were detected in levels of circulating anti-MPO antibodies between the experimental groups (Table 2-1). However, on day 6, mice that received the highest dose of LPS $(5 \mu \mathrm{g} / \mathrm{g})$ had a significantly lower anti-MPO titer compared to mice receiving anti-MPO alone (Table 2-1). No anti-MPO reactivity was detected in sera from mice treated with anti-BSA IgG and LPS $(0.5 \mu \mathrm{g} / \mathrm{g})$ or LPS alone.

Table 2-1: Anti-MPO antibody titers after passive transfer of anti-MPO IgG.

\begin{tabular}{|c|c|c|c|}
\hline \multirow[t]{2}{*}{ Groups } & \multirow[t]{2}{*}{$\mathrm{n}$} & \multicolumn{2}{|c|}{$\begin{array}{l}\text { Anti-MPO antibody titer } \\
\text { t AU (OD 405nm) of positive control) }\end{array}$} \\
\hline & & Day 1 & Day 6 \\
\hline anti-MPO & 5 & $85.2 \pm 10.0$ & $54.4 \pm 12.2$ \\
\hline $\begin{array}{l}\text { anti-MPO + } \\
5 \mu \mathrm{g} / \mathrm{g} \text { LPS }\end{array}$ & 5 & $77.4 \pm 13.5$ & $38.2 \pm 8.1^{*}$ \\
\hline $\begin{array}{l}\text { anti-MPO + } \\
0.5 \mu \mathrm{g} / \mathrm{g} \text { LPS }\end{array}$ & 5 & $83.6 \pm 2.7$ & $45.0 \pm 16.2$ \\
\hline $\begin{array}{l}\text { anti-MPO + } \\
0.05 \mu \mathrm{g} / \mathrm{g} \text { LPS }\end{array}$ & 4 & $82.5 \pm 3.1$ & $59.0 \pm 6.8$ \\
\hline $\begin{array}{l}\text { Anti-BSA + } \\
\text { LPS } 5 \mu \mathrm{g} / \mathrm{g}\end{array}$ & 5 & 0 & 0 \\
\hline
\end{tabular}

By day 1, hematuria had developed in all mice that received anti-MPO antibodies with or without LPS which persisted until the time of sacrifice at day 6 (Table 2-2). Most of these mice also had proteinuria and leukocyturia at day 6. Hematuria and leukocyturia measured at day 6 tended to be 
more severe in mice treated with anti-MPO $\operatorname{IgG}$ and $5.0 \mu \mathrm{g} / \mathrm{g}$ or $0.5 \mu \mathrm{g} / \mathrm{g}$ LPS, than in mice treated with anti-MPO IgG alone (Table 2-2). At day 6, serum creatinine and blood urea nitrogen levels were within normal range in all mice. No urinary abnormalities above background could be detected in mice treated with anti-BSA and $5.0 \mu \mathrm{g} / \mathrm{g}$ LPS, or with LPS alone (Table 2-2).

Table 2-2: Urinalysis and renal function at 6 days after disease induction

\begin{tabular}{ccccccc}
\hline Groups & $\mathbf{n}$ & Hematuria & Proteinuria & Leukocyturia & $\begin{array}{c}\text { Creatinine } \\
(\boldsymbol{\mu m o l} / \mathbf{L})\end{array}$ & $\begin{array}{c}\text { BUN } \\
(\mathbf{m m o l} / \mathbf{L})\end{array}$ \\
\hline $\begin{array}{cccccc}\text { anti-MPO } \\
\text { anti-MPO + }\end{array}$ & 5 & $2.7(1-3)$ & $2.8(2-4)$ & $0.8(0-2)$ & $15.6 \pm 7.0$ & $8.1 \pm 2.9$ \\
$5 \mu \mathrm{g} / \mathrm{g}$ LPS & 5 & $3.4^{*}(3-4)$ & $2.6(2-3)$ & $1.4(0-2)$ & $19.2 \pm 2.6$ & $8.0 \pm 0.9$ \\
$\begin{array}{c}\text { anti-MPO + } \\
0.5 \mu \mathrm{g} / \mathrm{g} \text { LPS }\end{array}$ & 5 & $3.8^{*}(3-4)$ & $3.2(2-4)$ & $2.6(2-3)$ & $18.2 \pm 2.4$ & $7.5 \pm 1.8$ \\
anti-MPO + & 4 & $2.3(1-3)$ & $2.3(1-3)$ & $1.0(1-2)$ & $21.3 \pm 2.6$ & $7.0 \pm 1.6$ \\
$0.05 \mu \mathrm{g} / \mathrm{g}$ LPS & & & & & & \\
Anti-BSA + & 5 & 0 & $1.4(1-2)$ & 0 & $18.5 \pm 2.4$ & $8.6 \pm 1.1$ \\
$5 \mu \mathrm{g} / \mathrm{g}$ LPS & & & & & & \\
\hline
\end{tabular}

Hematuria, proteinuria and leukocyturia were tested by dipstick and scored on 0-4+ scale. Values represent mean scores per group. Samples taken before antibody injection showed mean proteinuria $1.0+$, hematuria $0+$, and leukocyturia $0+$.

${ }^{*} \mathrm{p}<0.005$

\section{LPS AGGRAVATES ANTI-MPO IGG INDUCED NECROTIZING CRESCENTIC GLOMERULONEPHRITIS}

Intraperitoneal administration of anti-MPO IgG into WT mice induced a focal and segmental necrotizing crescentic glomerulonephritis in all subjected mice at day 6 , with a mean of $5.6 \%$ (range 2-10) crescentic and 9.0\% (range 7-12) necrotic glomeruli (Figure 2-1 and Figure 2-2). Administration of 5.0 or $0.5 \mu \mathrm{g} / \mathrm{g}$ LPS one hour after administration of anti-MPO IgG resulted in a marked increase in the severity of glomerulonephritis, as judged by the percentage of glomeruli containing crescents or fibrinoid necrosis. Addition of $5.0 \mu \mathrm{g} / \mathrm{g}$ LPS resulted in $20.4 \%$ (range 16-26) crescentic and $22.8 \%$ (range 12-36) necrotic glomeruli, whereas addition of 0.5 
$\mu \mathrm{g} / \mathrm{g}$ LPS resulted in 20.8\% (range 14-26) crescentic and 28.4\% (range 22-34) necrotic glomeruli (Figure 2-1 and Figure 2-2). Addition of $0.05 \mu \mathrm{g} / \mathrm{g}$ LPS did not lead to more severe disease compared to anti-MPO IgG alone, and administration of $100 \mu \mathrm{g} / \mathrm{g}$ anti-BSA $\operatorname{IgG}$ and $5.0 \mu \mathrm{g} / \mathrm{g}$ LPS, or LPS aloe, did not result in any histological abnormalities (Figure 2-1 and Figure 2-2).
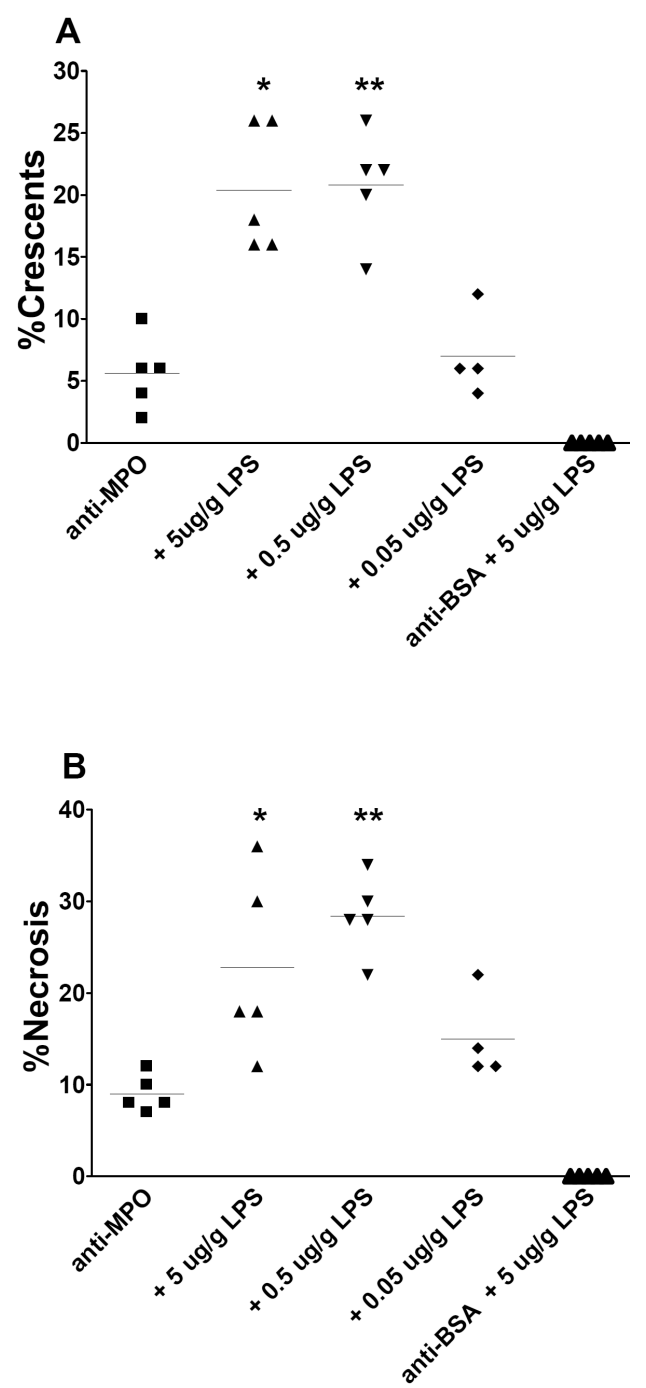

Figure 2-1: Effect of LPS on pathologic findings in C57Bl/ 6 mice 6 days after passive transfer of antiMPO IgG. a: Glomerular crescent formation expressed as a percentage of glomerular crescents in each animal. $* \mathrm{p}=0.0005, * * \mathrm{p}=0.0002$ compared to anti-MPO IgG alone b: Glomerular necrosis expressed as a percentage of glomeruli with necrosis in each animal. Horizontal lines represent mean percentages in each group. ${ }^{*} \mathrm{p}=0.02$, ** $\mathrm{p}=0.001$ compared to anti-MPO IgG alone.

Phenotypic analysis of the inflammatory cell infiltrate 6 days after administration of anti-MPO IgG demonstrated intraglomerular influx of CD45+ve leukocytes, the majority of which were found to be FA11+ macrophages (Figure 2-3, a and b, Table 2-3). A further increase in the number of glomerular infiltrating CD45+ leukocytes and FA11+ macrophages was observed upon addition of 5.0 or $0.5 \mu \mathrm{g} / \mathrm{g}$ LPS (Figure 2-3, c-f, Table 2-3). At this time point, numbers of intraglomerular CD3 $+\mathrm{T}$ cells and neutrophils were only slightly elevated in anti-MPO treated mice with or without LPS as compared to non-treated C57Bl/6 mice or mice that had received LPS $(5.0 \mu \mathrm{g} / \mathrm{g})$ alone. 

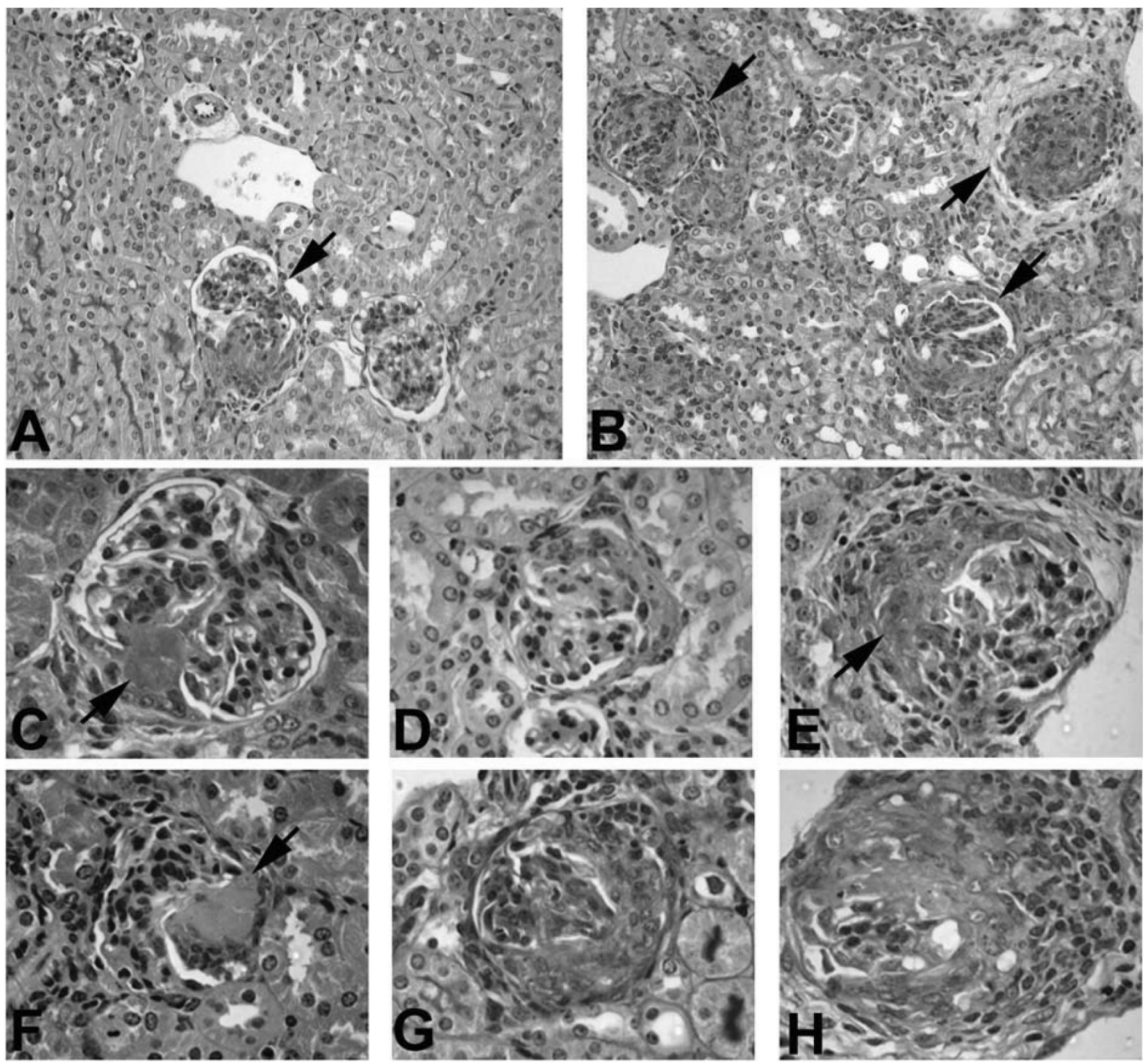

Figure 2-2: Effect of LPS on renal tissue injury in C57Bl/ 6 mice 6 days after passive transfer of anti-MPO IgG. a: Overview of renal tissue of a mouse that received anti-MPO IgG. One small crescentic glomerulus is shown (arrow). b: Overview of renal tissue in a mouse that received anti-MPO IgG and $0.5 \mu \mathrm{g} / \mathrm{g}$ LPS. Increased numbers of crescentic glomeruli are observed (arrows). c: Segmental fibrinoid necrosis in a glomerulus from a mouse that received anti-MPO IgG d: Small cellular crescent in mouse that received anti-MPO IgG. e: Glomerulus with segmental fibrinoid necrosis and crescent formation in a mouse that received anti-MPO IgG. f: Segmental fibrinoid necrosis in a glomerulus from a mouse that received anti-MPO IgG and $0.5 \mu \mathrm{g} / \mathrm{g}$ LPS. g: Large cellular crescent in mouse that received anti-MPO IgG and $0.5 \mu \mathrm{g} / \mathrm{g}$ LPS. h: Large cellular crescent with breaks in Bowman's capsule and periglomerular accumulation of inflammatory cells in a mouse that received anti-MPO IgG and $0.5 \mu \mathrm{g} / \mathrm{g}$ LPS. a,b,d,e,g,h: PAS stain. c,f: H\&E stain. Magnification a,b: x320; c-h: x400. 
Immunofluorescence revealed marked focal and segmental glomerular deposition of fibrin in anti-MPO treated mice with or without LPS at day 6 that corresponded with sites of segmental necrosis or crescent formation (Figure 2-3, i). In contrast, on day 6 glomerular staining for MPO and $\operatorname{IgG}$ was absent or scanty (Figure 2-3, g and h).
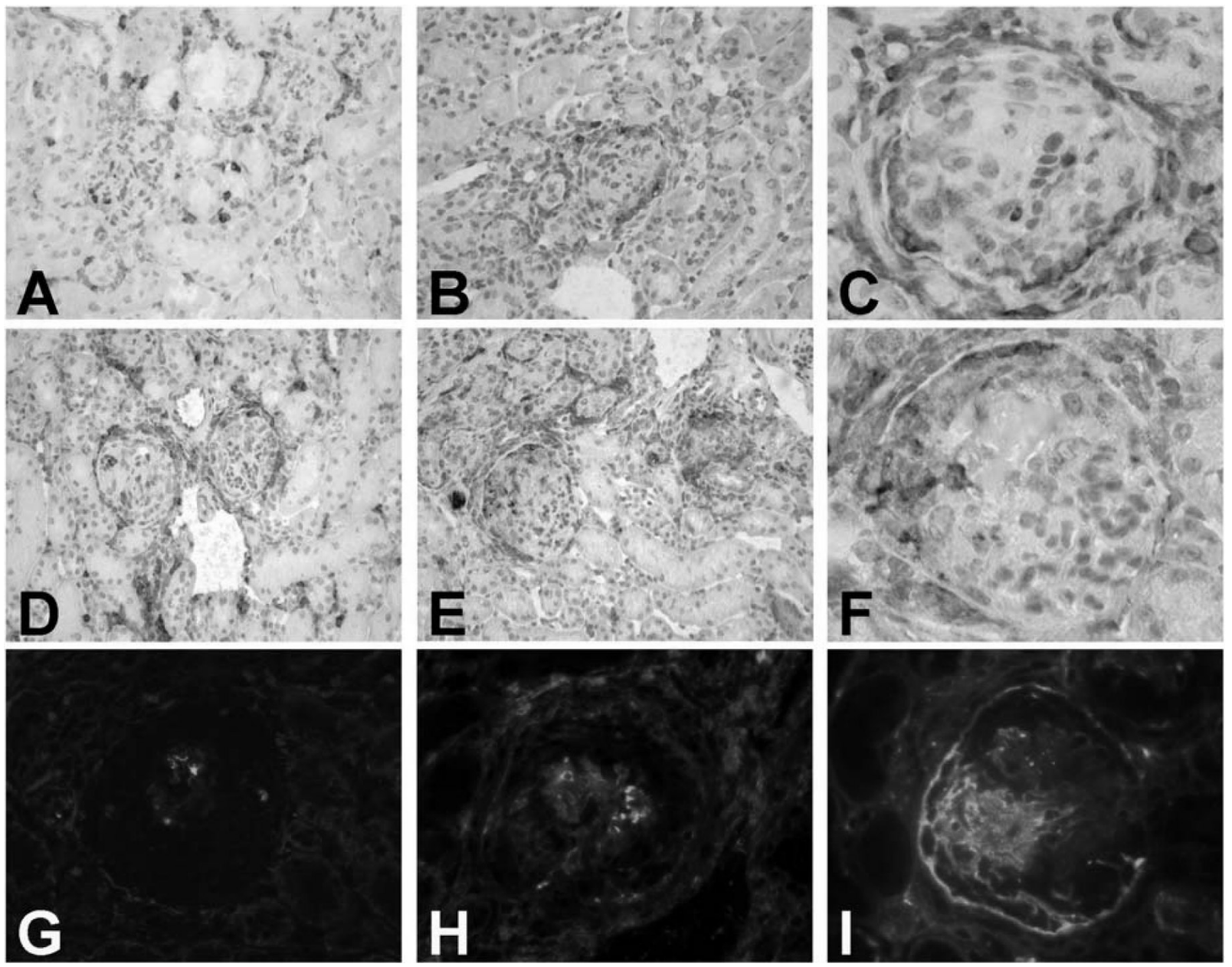

Figure 2-3: Effect of LPS on inflammatory cell recruitment in C57Bl/6 mice 6 days after passive transfer of antiMPO IgG. a: Moderate intraglomerular and interstitial infiltration of CD45 +ve leukocytes in a mouse that received anti-MPO IgG. b: Intra- and periglomerular influx of FA11 +ve macrophages in a mouse that received anti-MPO IgG. $\mathbf{c}$ and $\mathbf{d}$ : Marked intra- and periglomerular and interstitial influx of CD45 +ve leukocytes in a mouse that received anti-MPO IgG and $0.5 \mu \mathrm{g} / \mathrm{g}$ LPS. $\mathbf{e}$ and $\mathbf{f}$ : Marked intra- and periglomerular and interstitial influx of FA11 +ve macrophages in a mouse that received anti-MPO $\operatorname{IgG}$ and $0.5 \mu \mathrm{g} / \mathrm{g}$ LPS. g: Immunofluorescent staining for MPO in a mouse that received anti-MPO IgG and $0.5 \mu \mathrm{g} / \mathrm{g}$ LPS. h: Immunofluorescent staining for mouse IgG in a mouse that received anti-MPO IgG and $0.5 \mu \mathrm{g} / \mathrm{g}$ LPS. i: Immunofluorescent staining for fibrin in a mouse that received anti-MPO $\operatorname{IgG}$ and $0.5 \mu \mathrm{g} / \mathrm{g}$ LPS showing prominent staining corresponding to crescent formation. Magnification: a,b,d,e x200, c,f x630, g-i x400. 
Table 2-3: Immunophenotyping of intraglomerular inflammatory cell infiltrate at 6 days after disease induction

\begin{tabular}{|c|c|c|c|c|c|}
\hline Groups & $\mathbf{n}$ & $\begin{array}{c}\text { CD45 + } \\
\text { Leukocytes }\end{array}$ & $\begin{array}{c}\text { FA11 + } \\
\text { macrophages }\end{array}$ & $\begin{array}{l}\mathrm{CD} 3+ \\
\text { T cells }\end{array}$ & PMNs \\
\hline anti-MPO & 5 & $1.82 \pm 0.19$ & $1.34 \pm 0.13$ & $0.44 \pm 0.20$ & $0.39 \pm 0.10$ \\
\hline $\begin{array}{l}\text { anti-MPO + } \\
5 \mu \mathrm{g} / \mathrm{g} \text { LPS }\end{array}$ & 5 & $3.72 \pm 0.51^{*}$ & $2.24 \pm 0.07^{*}$ & $0.55 \pm 0.14$ & $0.48 \pm 0.19$ \\
\hline $\begin{array}{l}\text { anti-MPO + } \\
0.5 \mu \mathrm{g} / \mathrm{g} \text { LPS }\end{array}$ & 5 & $3.56 \pm 0.41 *$ & $2.45 \pm 0.19 *$ & $0.43 \pm 0.14$ & $0.39 \pm 0.10$ \\
\hline $\begin{array}{c}\text { anti-MPO + } \\
0.05 \mu \mathrm{g} / \mathrm{g} \text { LPS }\end{array}$ & 4 & $1.95 \pm 0.14$ & $1.48 \pm 0.22$ & $0.23 \pm 0.03$ & $0.21 \pm 0.07$ \\
\hline LPS $5 \mu \mathrm{g} / \mathrm{g}$ & 4 & $1.08 \pm 0.10$ & $0.44 \pm 0.15$ & $0.31 \pm 0.13$ & $0.22 \pm 0.08$ \\
\hline WT & 4 & $0.77 \pm 0.26$ & $0.36 \pm 0.20$ & $0.17 \pm 0.06$ & $0.09 \pm 0.07$ \\
\hline
\end{tabular}

${ }^{*} \mathrm{p}<0.0001$ compared to anti-MPO alone. Numbers represent mean numbers \pm SD of positive cells per glomerular cross section.

\section{LPS ENHANCES ANTI-MPO IGG INDUCED EARLY NEUTROPHIL RECRUITMENT}

At day 1, anti-MPO IgG treated mice showed increased glomerular influx of neutrophils that occasionally appeared as small neutrophilic aggregates (Figure $2-4, \mathrm{~b}$ ). This early glomerular influx of neutrophils was markedly enhanced upon addition of $0.5 \mu \mathrm{g} / \mathrm{g}$ LPS resulting in large neutrophilic aggregates (Figure 2-4, c). In contrast, injection of $0.5 \mu \mathrm{g} / \mathrm{g}$ LPS alone induced only a slight increase in the number of intraglomerular neutrophils, whereas glomerular neutrophil aggregation was not observed (Figure 2-4).

\section{LPS INCREASES SERUM MPO AND TNF $\alpha$ LEVELS}

In a separate experiment, the effect of a single dose of $5.0,0.5$, or $0.05 \mu \mathrm{g} / \mathrm{g}$ LPS on serum MPO and TNF $\alpha$ levels was determined. As shown in Figure 2-5, intraperitoneal administration of 5.0, 0.5 and $0.05 \mu \mathrm{g} / \mathrm{g}$ LPS led to a rise in serum TNF $\alpha$ levels one hour after injection. In case of the high $(5.0 \mu \mathrm{g} / \mathrm{g})$ and medium $(0.5 \mu \mathrm{g} / \mathrm{g})$ dose of LPS, this was followed by a rise in serum MPO levels at day 1. TNF $\alpha$ levels were undetectable at day 1 and day 6 after administration, whereas at the latter time point MPO levels had returned to baseline. Administration of saline alone did not lead to alterations in TNF $\alpha$ and MPO levels (data not shown). 

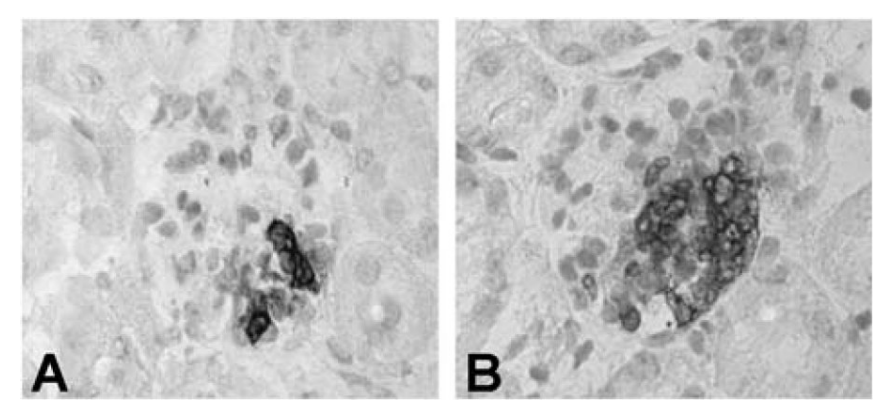

Figure 2-4: Effect of LPS on neutrophil influx and aggregation in $\mathrm{C} 57 \mathrm{Bl} / 6$ mice 1 day after passive transfer of anti-MPO IgG. a: Glomerulus from a mouse that received anti-MPO IgG showing segmental neutrophil influx and formation of a small neutrophilic aggregate. b: Glomerulus from a mouse that received anti-MPO and $0.5 \mu \mathrm{g} / \mathrm{g}$ LPS showing massive neutrophil influx and homotypic aggregation. $\mathbf{c}$ and $\mathbf{d}$ : Quantification of glomerular neutrophil influx (c) and the percentage of glomeruli that contained homotypic neutrophil aggregates $(d)$ in mice $(n=3$ in each group) that received LPS $(0.5 \mu \mathrm{g} / \mathrm{g})$ alone, antiMPO IgG alone or anti-MPO and LPS $(0.5 \mu \mathrm{g} / \mathrm{g})$. gcs, glomerular cross section. Magnification a and b: x400.* $\mathrm{p}=0.008$.
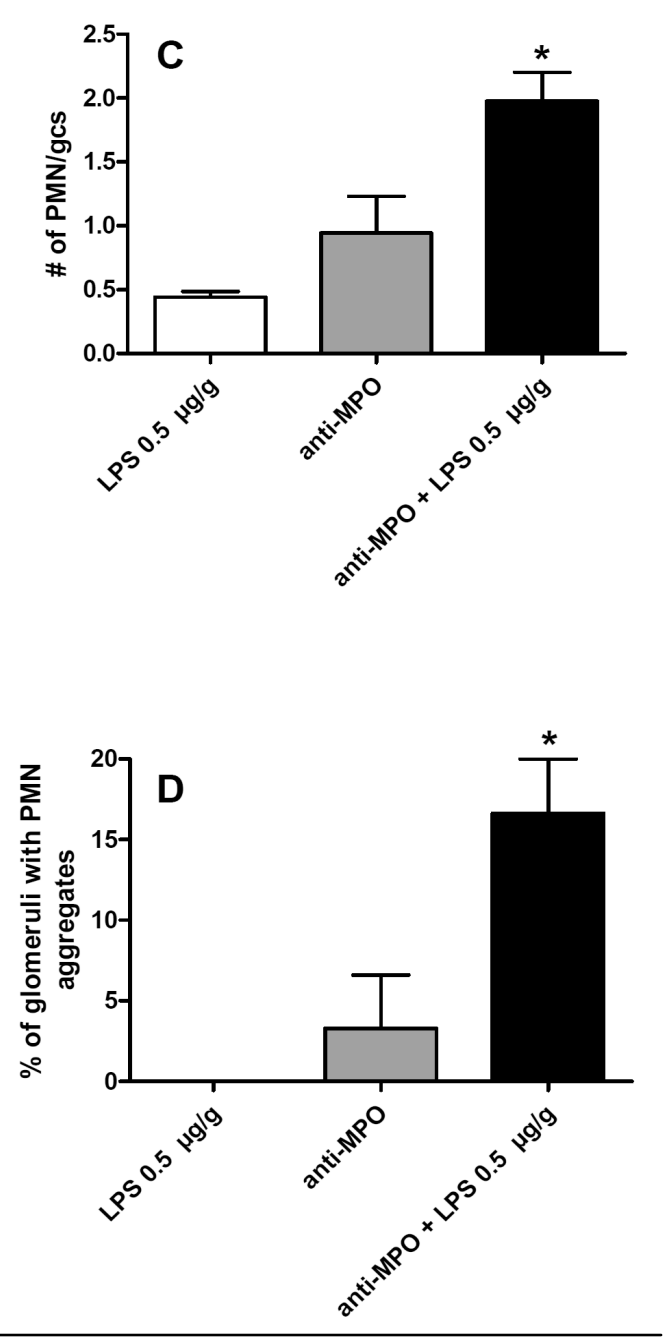


Figure 2-5: Serum MPO and $\mathrm{TNF} \alpha$ levels in $\mathrm{C} 57 \mathrm{Bl} / 6$ mice that received 0.05 (white bars), 0.5 (grey bars) or $5 \mu \mathrm{g} / \mathrm{g}$ (black bars) of LPS by intraperitoneal injection. The horizontal line indicates the mean serum MPO levels in serum samples taken before treatment. In these pretreatment samples, no $\mathrm{TNF} \alpha$ could be detected. 


\section{PretreatMent WiTH anti-TNF $\alpha$ ANTIBODY ATTENUATES \\ GLOMERULONEPHRITIS INDUCED BY ANTI-MPO IGG AND LPS}

To investigate the involvement of TNF $\alpha$ in the LPS-mediated aggravation of anti-MPO IgG induced glomerulonephritis, a neutralizing anti-murine TNF $\alpha$ antibody (TN3) was used. In these experiments, mice received an intraperitoneal injection of $500 \mu \mathrm{g}$ TN3 or isotype control on day 1, followed by administration of anti-MPO IgG and $0.5 \mu \mathrm{g} / \mathrm{g}$ LPS on day 0 . In both groups, mice developed hematuria and leukocyturia to a similar degree as measured by dipstick analysis. However, pathologic analysis at day 6 revealed that pretreatment with TN3 significantly attenuated glomerular crescent formation, whereas no difference was found in the extent of glomerular necrosis (Figure 2-6). In both groups, renal function on day 6 was unimpaired (BUN $7.8 \pm 1.4$ vs. $7.4 \pm 2.1$ and creatinine $19 . \pm 4.1$ vs. $19.3 \pm 1.9$ in TN3 and isotype control antibody treated mice, respectively).
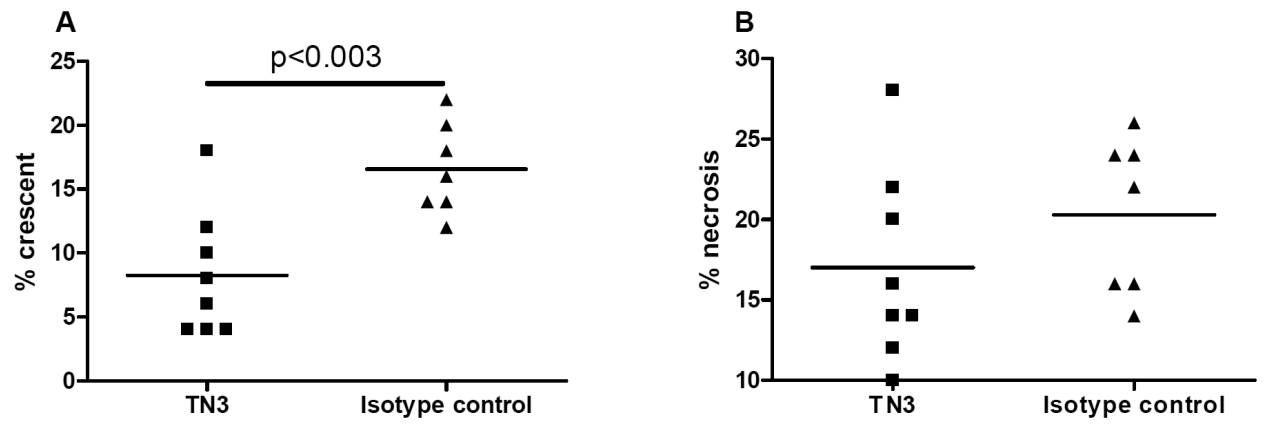

Figure 2-6: Effect of anti-TNF $\alpha$ treatment on necrotizing crescentic glomerulonephritis induced by anti-MPO IgG and LPS $(0.5 \mu \mathrm{g} / \mathrm{g})$. a: Quantification of glomerular crescent formation upon anti-TNF $\alpha$ or isotype control treatment. Glomerular crescent formation is expressed as a percentage of glomerular crescents in each animal. b: Quantification of glomerular necrosis upon anti-TNF $\alpha$ or isotype control treatment. Glomerular necrosis is expressed as a percentage of glomeruli with necrosis in each animal. 
Immunohistochemical analysis showed that pretreatment with TN3 significantly attenuated glomerular influx of CD45+ total leukocytes and FA11+ macrophages (Table 2-4). No differences were observed in intraglomerular numbers of neutrophils and T cells (Table 2-4). By immunofluorescence, glomerular IgG staining was absent or scanty in both groups.

Table 2-4: Immunophenotyping of intraglomerular inflammatory cell infiltrate at 6 days after anti-TNF $\alpha$ antibody or isotype control antibody (L2-3D9) treatment

\begin{tabular}{cccccc}
\hline Groups & $\mathbf{n}$ & $\begin{array}{c}\text { CD45 }+ \\
\text { Leukocytes }\end{array}$ & FA11 & CD3 + & PMNophages \\
& & & T cells & \\
\hline $\begin{array}{c}\text { anti-MPO }+ \\
0.5 \mu \mathrm{g} / \mathrm{g} \text { LPS } \\
+\mathrm{L} 2-3 \mathrm{D} 9\end{array}$ & 8 & $3.03 \pm 0.52^{*}$ & $2.26 \pm 0.62^{*}$ & $0.33 \pm 0.14$ & $0.37 \pm 0.10$ \\
$\begin{array}{l}\text { anti-MPO }+ \\
0.5 \mu \mathrm{g} / \mathrm{g} \text { LPS }\end{array}$ & 7 & $2.23 \pm 0.41$ & $1.53 \pm 0.29$ & $0.37 \pm 0.08$ & $0.35 \pm 0.14$ \\
$+\mathrm{TN} 3$ & & & & & \\
\hline
\end{tabular}

${ }^{*} \mathrm{p}<0.005$ compared to anti-MPO $+0.5 \mu \mathrm{g} / \mathrm{g}$ LPS $+\mathrm{TN} 3$. Numbers represent mean numbers \pm SD of positive cells per glomerular cross section.

\section{ANTI-MPO IGG INDUCES SUPEROXIDE ANION PRODUCTION IN TNF $\alpha$-PRIMED PERITONEAL EXUDATE CELLS IN VITRO}

The capacity of anti-MPO antibodies to induce neutrophil activation in vitro was determined using the SOD inhibitable ferricytochrome $\mathrm{C}$ assay. Incubation of unprimed peritoneal exudate cells with normal mouse $\operatorname{IgG}$ or anti-MPO IgG did not induce superoxide production above background (data not shown). However, when peritoneal exudate cells were pretreated with 10 $\mathrm{ng} / \mathrm{ml}$ murine TNF $\alpha$ for 10 minutes, incubation with anti-MPO IgG resulted in a modest but significant increase in superoxide production as compared to normal mouse $\operatorname{IgG}$ or buffer alone (Figure 2-7, a and b). Importantly, anti-MPO IgG did not induce superoxide anion production in TNF $\alpha$ primed, MPO deficient peritoneal exudate cells (Figure 2-7, a and c). PMA induced superoxide anion production in these cells was, however, similar to that observed in wildtype peritoneal exudate cells (Figure 2-7, a). 




\section{B}

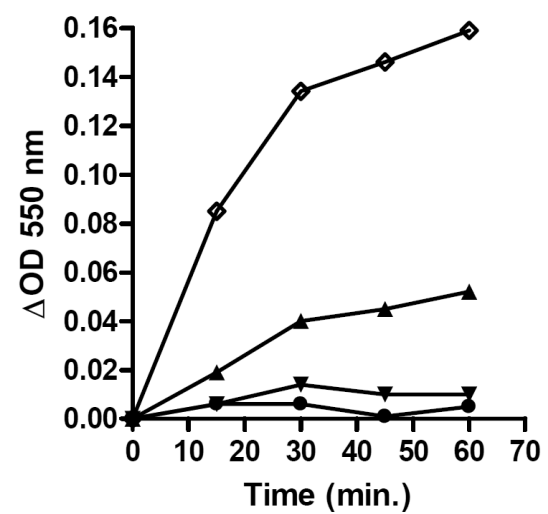

C



Figure 2-7a: Superoxide anion production in TNF $\alpha(10 \mathrm{ng} / \mathrm{ml})$ primed peritoneal exudate cells $\left(4 \times 10^{5}\right.$ cells/well) derived from wildtype $\mathrm{C} 57 \mathrm{Bl} / 6$ or $\mathrm{MPO}^{-/-}$mice as measured by the SOD inhibitable ferricytochrome $\mathrm{C}$ assay. AntiMPO IgG $(250 \mu \mathrm{g} / \mathrm{ml})$ induces significant superoxide anion production in TNF $\alpha$ primed peritoneal exudate cells from wildtype C57Bl/6 mice (black bars) as compared to normal mouse $\operatorname{IgG}(250 \mu \mathrm{g} / \mathrm{ml})$ or buffer alone. In contrast, no superoxide anion production was detected in TNF $\alpha$ primed peritoneal exudate cells from $\mathrm{MPO}^{-/-}$mice (white bars) upon stimulation with anti-MPO IgG. Results represent mean $\triangle \mathrm{OD} 550 \mathrm{~nm} \pm \mathrm{SD}$ of 3 to 4 experiments performed in triplicate. ${ }^{*} \mathrm{p}=0.02$ compared to anti-MPO IgG on MPO deficient cells. $\mathbf{b}$ and $\mathbf{c}$ : Time course of superoxide anion production in response to PMA $(\diamond, 200 \mathrm{ng} / \mathrm{ml})$, anti-MPO $\operatorname{IgG}(\boldsymbol{\Lambda}, 250 \mu \mathrm{g} / \mathrm{ml})$, normal mouse $\operatorname{IgG}(\boldsymbol{\nabla}, 250 \mu \mathrm{g} / \mathrm{ml})$ or buffer alone $(\bullet)$ using TNF $\alpha$ primed peritoneal exudates cells from wildtype C57Bl/6 (b) or $\mathrm{MPO}^{-/-}$(c) mice. Results represent mean $\Delta \mathrm{OD} 550 \mathrm{~nm}$ from single experiments performed in triplicate. 


\section{Discussion}

In the present study, three main findings are reported that are relevant to the pathogenesis of MPO-ANCA induced glomerulonephritis. First, it was demonstrated that systemic administration of bacterial LPS markedly aggravates anti-MPO induced NCGN. This effect was dependent on the dose of LPS administered and appeared to be related to LPS-induced circulating levels of TNF $\alpha$ and MPO. Second, LPS-mediated aggravation of anti-MPO IgG induced glomerulonephritis was attenuated, but not prevented, by pretreatment with neutralizing antiTNF $\alpha$ antibodies. Finally, in vitro experiments showed that murine anti-MPO $\operatorname{IgG}$ induces an oxidative burst in TNF $\alpha$-primed peritoneal exudate cells, an observation that is consistent with human data showing ANCA mediated neutrophil activation.

In our studies, glomerulonephritis induction by anti-MPO IgG alone is less severe compared to similar groups of mice reported in the companion paper by Xiao et al. The most plausible explanation for this difference in disease severity is the route of injection of the anti-MPO antibodies. In our studies, anti-MPO antibodies were injected intraperitoneally whereas in the companion paper the antibodies were administered intravenously. This could potentially lead to different levels of circulating anti-MPO antibodies but we have not formally tested this. Another explanation may be that the antibody batches used in these two studies differed in the amount of pathogenic anti-MPO antibodies. Importantly, however, in both studies, transfer of anti-MPO antibodies induced crescentic glomerulonephritis in all of the tested mice which emphasizes the pathogenic potential of these autoantibodies.

Our observation that LPS dose-dependently aggravates anti-MPO induced glomerulonephritis provides support for the hypothesis that ANCA and proinflammatory stimuli act synergistically in inducing full-blown disease in humans. With respect to the mechanisms underlying this effect several possibilities may be considered and will be discussed below.

First, as shown here and by many others, systemic administration of LPS results in an immediate, transient increase in serum TNF $\alpha$ levels. From in vitro experiments on human PMNs, it is well known that priming with TNF $\alpha$ is necessary for the induction of a respiratory burst by sera or purified IgG from ANCA-positive patients $[9-13,73]$. We confirmed this in our mouse model by showing that anti-MPO IgG induced an oxidative burst in peritoneal exudate cells only after pretreatment with TNF $\alpha$, and only when those cells were obtained from MPO-competent mice. Second, we observed that administration of LPS synergistically increased anti-MPO IgG induced 
early glomerular neutrophil recruitment. The number of infiltrating PMNs was significantly increased in anti-MPO treated mice with or without LPS that were sacrificed one day after disease induction. These results suggest an important role for PMNs in the initiation of antiMPO IgG-induced disease. The number of lesional PMNs had decreased on day 6 which most likely is a reflection of the progression of the inflammatory response that is dominated by macrophages. This is in line with in vitro data, demonstrating an important role for priming of endothelial cells with TNF $\alpha[16]$ or LPS[81] in anti-MPO IgG induced neutrophil adhesion. Subsequently, these adherent neutrophils may become activated by MPO-ANCA causing endothelial cell injury [15]. Despite aggravation of renal injury by LPS as observed by histopathological analysis, renal function in these mice was not impaired. Apparently, the number of affected glomeruli was not sufficient to cause elevations in serum creatinine and BUN levels.

The requirement for TNF $\alpha$ priming in ANCA-mediated neutrophil activation is in part explained by the observation that primed neutrophils translocate the ANCA antigens to their cell surface making them accessible for interaction with the autoantibodies. In the case of MPO-ANCA, studies have clearly demonstrated that expression of MPO is essential for MPO-ANCA mediated neutrophil activation because neutrophils obtained from MPO deficient individuals are nonresponsive [82]. However, inconsistent data exist on whether MPO-ANCA mediated neutrophil activation involves MPO expressed on the cell surface of primed neutrophils. Whereas some authors describe an enhanced surface expression of MPO on primed neutrophils, others have been unable to demonstrate this effect $[9,11,12,73]$. In preliminary experiments using flow cytometry, we have not been able to convincingly demonstrate surface MPO expression on either peripheral neutrophils from LPS-treated mice or peritoneal exudate cells treated with LPS in vitro. One possibility is that priming induces cell surface MPO expression in minute amounts that are below the detection limits of our assay system but this clearly needs further investigation. Alternatively, other mechanisms may be involved in MPO-ANCA mediated neutrophil activation. Studies by Hess and colleagues have shown that resting human neutrophils exposed to supernatants of degranulated autologous neutrophils expressed MPO, but not $\operatorname{Pr} 3$, on their cell surface and became responsive to anti-MPO autoantibodies [11]. Interestingly, high levels of circulating MPO correlate with disease severity in human MPO-ANCA associated glomerulonephritis [71]. In the present study, we found that doses of LPS that aggravated antiMPO IgG induced glomerulonephritis also gave rise to increased levels of circulating MPO. Based on these observations we speculate that circulating MPO, in the presence of high levels of 
anti-MPO antibodies, may disperse MPO-ANCA mediated activation to resting neutrophils resulting in amplification of the inflammatory response.

As described above, ANCA induced neutrophil activation is enhanced upon priming with proinflammatory stimuli, in particular TNF $\alpha$. This is a potent pro-inflammatory cytokine produced by many cell types, and there is mounting evidence that TNF $\alpha$ plays a major role in glomerular inflammation and scarring [83]. The actions of TNF $\alpha$ are numerous and include the stimulation of release of other cytokines and chemokines, and the induction of adhesion molecule expression on the endothelium [84]. To directly investigate the contribution of TNF $\alpha$ on the LPS-mediated aggravation of anti-MPO IgG induced glomerulonephritis, we treated mice with a neutralizing anti-TNF $\alpha$ antibody prior to disease induction. It was found that TNF $\alpha$-inhibition reduced glomerular crescent formation whereas development of glomerular necrosis was unaffected. These results are in agreement with studies in TNF $\alpha$-deficient mice, in which the development of accelerated anti-glomerular basement antibody (GBM) glomerulonephritis is also attenuated but not completely prevented [85]. On the other hand, complete prevention of glomerular crescent formation has been observed in a rat model of anti-GBM glomerulonephritis upon treatment with the soluble TNF $\alpha$ receptor P55 [86]. Our observations suggest that, as yet unknown, TNF $\alpha$ independent effects also play a role in the LPS-mediated aggravation of anti-MPO IgG induced glomerulonephritis but this clearly needs to be investigated in more detail.

In conclusion, the results of the present study are in agreement with the hypothesis that in ANCA-associated diseases, autoantibody mediated effects and proinflammatory signals synergize in causing vascular inflammation by promoting neutrophil adhesion to the endothelium and enhancing neutrophil activation. Furthermore, our findings indicate that therapeutic strategies aimed at neutralizing TNF $\alpha$ bioactivity could be beneficial in the treatment of ANCA- associated diseases. In fact, such clinical studies have already been initiated and in a first preliminary publication, promising results have been reported [87]. 

Chapter 3 - Tumor necrosis factor-alpha bioactivityinhibiting therapy in ANCA-associated vasculitis clinical and experimental considerations

Huugen D, Cohen Tervaert JW, Heeringa P. Clin. J. Am. Soc. Nephrol. 2006 Sep;1:1100-1107. 


\section{Abstract}

Wegener's granulomatosis, microscopic polyangiitis, idiopathic necrotizing crescentic glomerulonephritis, and ChurgStrauss syndrome are associated with the presence of anti-neutrophil cytoplasmic autoantibodies (ANCA) with specificity for myeloperoxidase (MPO-ANCA) or proteinase 3 (PR3-ANCA). Current therapy consists mainly of corticosteroids and cyclophosphamide, but since this treatment regimen is associated with considerable morbidity, other treatment modalities remain desirable.

There is compelling evidence that tumor necrosis factor-alpha (TNF $\alpha$ ) plays an important role in the pathogenesis of ANCA-associated vasculitis. Consequentially, inhibition of TNF $\alpha$ bioactivity potentially results in attenuation of disease. In this review, we discuss whether TNF $\alpha$ bioactivity-inhibiting drugs are useful in the treatment of ANCAassociated vasculitis. The results of in vitro and in vivo experiments, as well as clinical studies are evaluated. Although the importance of TNF $\alpha$ during lesion development is evident, clinical trials using TNF $\alpha$ blockers in patients with ANCA-associated vasculitis give mixed results. Importantly, in a large-scale randomized trial treatment with etanercept was found to be not effective and resulted in an excess of treatment-related morbidity. It remains to be investigated whether inhibition of TNF $\alpha$ bioactivity is effective in a subgroup of patients. 


\section{Introduction}

Wegener's granulomatosis (WG), microscopic polyangiitis (MPA), idiopathic necrotizing crescentic glomerulonephritis (NCGN), and Churg-Strauss syndrome (CSS) are associated with anti-neutrophil cytoplasmic autoantibodies (ANCA) [5]. They are not only widely used as serum markers for the diagnosis of systemic vasculitis, but also for follow-up, since ANCA-levels predict disease reactivation in most patients [7]. In vasculitis and glomerulonephritis, the main ANCA-antigens are the myeloid enzymes myeloperoxidase (MPO) and proteinase 3 (PR3), but in a minority of patients, ANCA are specific for other neutrophil proteins such as elastase.

To date, therapy for ANCA-associated disease consists mainly of immunosuppression with highdose glucocorticoids and cyclophosphamide during the induction phase and azathioprine in combination with low-dose steroids as maintenance therapy. This therapeutic regimen is associated with considerable morbidity, and often proves to be insufficient for the induction of a sustained remission, since most patients develop relapsing disease activity during follow-up. Consequently, renal, pulmonary, and/or other organ damage is encountered [88].

In various immune-mediated diseases, including rheumatoid arthritis [89-91], Crohn's disease [92] and ankylosing spondylitis [93, 94], the efficacy of TNF $\alpha$-inhibiting treatment is well established. Also in several forms of vasculitis, such as Takayasu arteritis [95] and Behçet's disease [96-98], there is evidence that inhibition of TNF $\alpha$ bioactivity-inhibition is beneficial.

Here, we review the literature on the role of TNF $\alpha$ in ANCA-associated vasculitides. In vitro studies as well as in vivo experimental data and clinical trials on the feasibility of TNF $\alpha$ bioactivity inhibition will be discussed, as well as their consequences for the future use of this treatment modality in patients with ANCA-associated disease.

\section{The role of TNF $\alpha$ in ANCA-associated vasculitis - in vitro observations}

There is a considerable amount of data obtained from in vitro experiments pointing towards a role for TNF $\alpha$ in the pathogenesis of ANCA-associated disease. Importantly, ANCA-induced neutrophil activation is greatly enhanced by $\mathrm{TNF} \alpha$, leading to an increased release of oxygen radicals and toxic granule constituents [9-13]. The exact mechanism of this effect is uncertain, but several reports demonstrate an increased presence of MPO and/or PR3 on the outer membrane of neutrophils after incubation with TNF $\alpha[11,12,99,100]$. This increases the availability of the 
ANCA-antigens for binding of the autoantibodies. In addition, neutrophil priming with TNF $\alpha$ causes upregulation of various molecules involved in adhesion of neutrophils to the endothelium [101], and it has been convincingly demonstrated that in particular one of these molecules, the $\beta_{2}$ integrin CD11b/CD18 (CR3), is critically involved in ANCA-induced neutrophil activation [73]. In vitro, TNF $\alpha$ pretreatment of endothelial cells makes them more susceptible to damage induced by incubation with ANCA-stimulated neutrophils, in particular if those neutrophils are also preincubated with TNF $\alpha[15,102]$. Moreover, pretreatment of human umbilical cord vascular endothelial cells with TNF $\alpha$ is necessary for the establishment of firm adhesion of ANCAstimulated neutrophils to those cells [16].

Taken together, in vitro data point towards a mechanism in which TNF $\alpha$ and ANCA together induce the activation of neutrophils. These activated neutrophils attach to the endothelium, release their toxic granule constituents and oxygen radicals, and thus cause vascular damage.

\section{TNF $\alpha$ in animal models of autoimmune crescentic}

\section{glomerulonephritis}

In crescentic glomerulonephritis, the role of $\mathrm{TNF} \alpha$ has been investigated most thoroughly in animal models of anti-glomerular basement membrane (GBM) glomerulonephritis. In these models, heterologous antibodies to the GBM are administered to mice or rats, respectively, either with (in the accelerated model) or without (in the heterologous model) preceding immunization with unspecific heterologous antibodies. This results in crescentic glomerulonephritis characterized by massive early neutrophil-influx and an abundance of immune complexes.

Le Hir et al. studied the role of TNF $\alpha$ in the accelerated model of anti-GBM glomerulonephritis using TNF $\alpha$-knockout $\left(\mathrm{TNF}^{-/}\right)$mice, and found that TNF $\alpha$-deficiency led to attenuation, but no complete inhibition, of disease as reflected by delayed onset of proteinuria and attenuation of histopathological and immunohistochemical alterations [85]. Importantly, no significant decrease in early neutrophil influx could be observed in the $\mathrm{TNF}^{-1-}$-mice.

Whereas $\mathrm{TNF}^{-/-}$-mice are only partially protected from disease in the accelerated model of antiGBM GN, treatment with daily doses of a soluble TNF $\alpha$ receptor (sTNFr) from day -1 onwards, completely prevents the development of crescents in the heterologous rat model of anti-GBM GN [86]. An explanation for the discrepancy between this study and the study in $\mathrm{TNF} \alpha^{-/-}$-mice by le Hir et al. is not provided, but it is conceivable that TNF $\alpha$ plays a more 
profound role in the heterologous, neutrophil-dependent, than in the accelerated model of antiGBM disease. Alternatively, TNF $\alpha^{-/}$-mice, due to their lifelong lack of endogenous TNF $\alpha$, may have developed a compensatory mechanism for this deficiency, thus increasing the levels of alternative proinflammatory cytokines that play a role in the development of anti-GBM GN. Interestingly, in the rat model, treatment of established anti-GBM disease (from day +4 onwards) with sTNFr resulted in a marked attenuation of disease 10 days after disease induction, reflected by reduced proteinuria, crescent formation, fibrinoid necrosis, and glomerular influx of monocytes and cytotoxic T-cells [86]. This implies that TNF $\alpha$ does not only play a role in the initial phase of disease development, but also in maintaining the disease, a notion that is further supported by a more recent study from the same group, in which the effect of a TNF $\alpha$-inhibiting monoclonal antibody (moAb) on the course of (heterologous) anti-GBM GN is studied in rats that are followed for as long as 28 days [103]. In this study, treatment of established anti-GBM GN with anti-TNF $\alpha$ moAb from day 4 or day 14 until sacrifice at day 28 significantly attenuated disease as measured by glomerular and tubulointerstitial scarring and serum creatinine level.

Investigation of the role of TNF $\alpha$ in ANCA-associated GN has long been hampered by the lack of an animal model in which the pathogenicity of ANCA could be convincingly demonstrated. In a mouse model of spontaneous crescentic glomerulonephritis in SCG/Kj mice [56], TNF $\alpha$ levels were shown to be elevated compared to healthy C57Bl/6 mice [104]. Although MPO-ANCA are detected in these mice, they also display massive glomerular immune complex depositions and elevated titers of anti-nuclear autoantibodies [60]. Therefore, the contribution of MPO-ANCA to the pathology observed in this model is questionable.

An alternative mouse model of MPO-ANCA associated vasculitis is provided by Xiao and colleagues, showing that transfer of $\operatorname{IgG}$ or splenocytes from murine MPO-immunized MPOknockout $\left(\mathrm{MPO}^{-/}\right)$mice to wildtype or immune-deficient mice induces disease manifestations similar to those observed in human MPO-ANCA associated disease [43]. Using the passive transfer model of anti-MPO IgG-induced glomerulonephritis, we recently demonstrated that a 'second hit' with lipopolysaccharide (LPS) significantly increased the severity of anti-MPO IgG induced lesions [105]. Furthermore, in this accelerated model of MPO-ANCA induced GN, we found that administration of a single dose of TNF $\alpha$ bioactivity-inhibiting moAb one day prior to disease induction led to a significant decrease, but no complete inhibition, of renal disease as measured by the degree of urinary abnormalities and the percentage of crescentic glomeruli. This study suggests that TNF $\alpha$ plays an important, although not pivotal, role in the pathogenesis of 
NCGN induced by anti-MPO IgG and LPS. However, the fact that these results are obtained after preemptive anti-TNF $\alpha$ treatment, might limit their applicability for the clinical situation. Interestingly, the effect of TNF $\alpha$ bioactivity-inhibiting therapy was recently also investigated in a novel rat model of MPO-ANCA-associated NCGN. In this model, the immunization of WKY/NCrlBR rats with human MPO leads to the generation of anti-human MPO antibodies that cross-react with rat MPO, resulting in pulmonary vasculitis and pauci-immune NCGN in some animals [49]. Additionally, treatment of established disease with a TNF $\alpha$-inhibiting moAb strongly reduced albuminuria and completely reversed crescent formation [106].

Taken together, data obtained from animal models of anti-GBM as well as MPO-ANCAassociated NCGN clearly indicate that TNF $\alpha$ plays an important role in disease induction and progression and suggest a beneficial role for TNF $\alpha$ bioactivity inhibition in humans. Due caution should, however, be taken when extrapolating the results from animal experiments to the human situation.

\section{TNF $\alpha$-inhibition - human studies}

The efficacy of two TNF $\alpha$-inhibiting drugs has been investigated in patients with ANCAassociated NCGN. Firstly, etanercept (Enbrel®) is a fusion protein of two p75 subunits of the TNF $\alpha$ receptor, linked to the Fc portion of human IgG1. Secondly, infliximab (Remicade ${ }^{\circledR}$ ) is a chimeric IgG1 monoclonal antibody that binds and inhibits soluble as well as membrane-bound TNF $\alpha$. The efficacy of both treatments in patients with rheumatoid arthritis seems to be comparable [107], but in patients with Crohn's disease, etanercept, in contrast to infliximab, seems to be not effective [108]. The role of adalimumab (Humira $\left.{ }^{\circledR}\right)$, a novel, fully human TNF $\alpha$ inhibiting monoclonal antibody [109], that has been shown to be effective in rheumatoid arthritis and Crohn's disease when the response to etanercept or infliximab is lost $[110,111]$, in the treatment of ANCA-associated vasculitis remains to be established.

From the in vitro and in vivo studies on the role of TNF $\alpha$ in ANCA-associated vasculitis discussed above, it may be hypothesized that an effect of anti-TNF $\alpha$ treatment would be the result of inhibition of TNF $\alpha$-induced preactivation of PMNs. This would lead to decreased membrane expression of the ANCA-antigens, what makes it impossible for the autoantibodies to exert their pathogenic effect. 
Several clinical studies provide indirect evidence for a role of TNF $\alpha$ in ANCA-associated vasculitis. First, plasma levels of TNF $\alpha$ are increased in patients with ANCA-associated glomerulonephritis, and increased amounts of TNF $\alpha$ can be found by immunohistochemistry [112]. Second, the fractional excretion of TNF $\alpha$ is increased in patients with ANCA-associated glomerulonephritis, suggesting local production by renal cells or infiltrating leukocytes [113]. Third, response to treatment with intravenous immunoglobulins is accompanied by a decrease in serum TNF $\alpha$ level [114]. Finally, it has been shown in rheumatoid arthritis that TNF $\alpha$ is pivotally involved in a tightly regulated network of proinflammatory cytokines, and is responsible for the increased production of the interleukins 1, 6, and 8, and granulocyte-macrophage colonystimulating factor (reviewed in [115]). Consequently, inhibition of TNF $\alpha$ bioactivity in patients with ANCA-associated vasculitis would also result in decreased levels of those cytokines, and thereby decrease vascular inflammation.

The human studies on TNF $\alpha$ bioinhibition in ANCA-associated vasculitis are briefly summarized in Table 3-1. In the first study, infliximab was added in an uncontrolled fashion to standard treatment consisting of corticosteroids and cyclophosphamide in six patients with treatmentresistant Wegener's granulomatosis [116]. This led to remission in five cases, lasting for six to 24 months. Addition of infliximab to standard immunosuppressive therapy also led to remission in a more recently published case of Wegener's disease [117].

A large, prospective, but also uncontrolled, trial among patients with MPO- or PR3-ANCApositive vasculitis has been published in 2004 [87]. Addition of infliximab to standard therapy at initial presentation, during relapse, or during persistent disease activity, was followed by a clinical response in 28 out of 32 patients, as measured with the Birmingham Vasculitis Activity Score [118] and reflected by a decrease in C-reactive protein, serum creatinine, and required steroid dose. Unfortunately, however, many side-effects were observed, including several cases of infection, thrombotic events and a case of B cell lymphoma, complicating clinical response. In addition, despite ongoing treatment with infliximab, relapses were observed frequently. This suggests an escape mechanism, that might be partly mediated by the development of antiinfliximab antibodies [91, 119-122], although this was not investigated in detail. In an accompanying paper, the administration of infliximab significantly improved endothelial function in 10 patients with active ANCA-associated disease, as measured by endothelium-dependent vasodilation, and also in these patients, a clinical response was observed [123].

Until now, studies have mainly been focusing on the addition of infliximab to standard immunosuppressive therapy, and there is only limited information on infliximab replacing 
standard treatment. In an uncontrolled study on 10 patients with conventional therapy-resistant systemic vasculitis, seven of which had WG, Bartolucci et al. found that treatment with corticosteroids and infliximab led to a considerable resolution of disease symptoms and corticosteroid requirement at 42 days and six months [124]. Although limited in size and uncontrolled, this study encourages further research into the replacement of conventional treatment by infliximab in individuals with ANCA-associated vasculitis that fail to enter remission under standard therapy. A case report, in which remission induction was achieved in a patient with MPA after primary treatment with prednisolone, cotrimoxazole/trimethoprim and 4 infusions of infliximab, supports this notion [125].

Table 3-1: Human studies on TNF $\alpha$ inhibition

\begin{tabular}{|c|c|c|c|c|c|c|}
\hline Ref. & Study type & $\mathbf{n}$ & Diagnosis (n) & Intervention & $\begin{array}{c}\text { Remission } \\
\text { (n) }\end{array}$ & $\begin{array}{c}\text { Follow-up } \\
\text { (months) }\end{array}$ \\
\hline [116] & $\begin{array}{c}\text { Uncontrolled } \\
\text { trial }\end{array}$ & 6 & WG & CS, CTX, IFX & 5 & $6-24$ \\
\hline [117] & Case study & 1 & WG & CS, CTX, IFX & 1 & 7 \\
\hline [87] & $\begin{array}{c}\text { Uncontrolled } \\
\text { trial }\end{array}$ & 32 & WG/MPA & $\begin{array}{l}\text { CS, S/T, CTX, AZ, } \\
\text { MTX, MMF, IFX }\end{array}$ & 28 & Max. 12 \\
\hline [124] & $\begin{array}{l}\text { Uncontrolled } \\
\text { trial }\end{array}$ & 10 & $\begin{array}{l}\text { WG (7)/ } \\
\text { other (3) }\end{array}$ & $\mathrm{CS}, \mathbf{I F X}^{*}$ & $\begin{array}{l}\text { CR: } 5 \\
\text { PR: } 5\end{array}$ & 6 \\
\hline [125] & Case report & 1 & $\begin{array}{l}\text { Pauci- } \\
\text { immune } \\
\text { NCGN }\end{array}$ & $\mathrm{CS}, \mathrm{S} / \mathrm{T}, \mathbf{I F X}$ & 1 & 12 \\
\hline [126] & $\begin{array}{l}\text { Controlled } \\
\text { trial }\end{array}$ & 181 & WG & $\begin{array}{l}\text { CS, CTX or MTX, } \\
\text { AZ, S/T, ETA or } \\
\text { placebo }\end{array}$ & $\begin{array}{c}\text { Exp: } \\
\text { 69.7\% } \\
\text { Ctrl: } 75.3 \%\end{array}$ & $\begin{array}{l}>9 \text { (mean: } \\
27 \text { months) }\end{array}$ \\
\hline
\end{tabular}

Abbreviations: CS, corticosteroids; CTX, cyclophosphamide; IFX, infliximab; S/T, sulfamethoxazole/trimethoprim; AZ, azathioprine; MTX, methotrexate; MMF, mycophenolate mofetil; CR, complete remission; PR, partial remission; ETA, etanercept

\footnotetext{
* Two patients with severe disease received additional therapy with CTX, and MTX and MMF, respectively
} 
In contrast to data on the use of infliximab, the effect of etanercept in patients with WG is less promising. In the only double-blind placebo-controlled multicenter trial on the use of TNF $\alpha$ inhibiting therapy in ANCA-associated vasculitis presented thus far, the Wegener's Granulomatosis Etanercept Trial (WGET) Research Group investigated the efficacy of the addition of etanercept to standard therapy [126]. Patients with limited and severe disease, either newly diagnosed or relapsing, were enrolled. Standard treatment for limited and severe disease consisted of glucocorticoids, and methotrexate or cyclophosphamide, respectively; etanercept or placebo was randomly added to this as experimental treatment. Between the etanercept and placebo group, no significant differences were found in remission rates, disease flares, and disease activity, damage, or Quality of Life scores. Remarkably, during follow-up, six cases of cancer were identified in the etanercept group, whereas no such events were seen in the control group. No significant differences were found in the incidence and severity of other adverse events.

The results of the etanercept study are surprisingly negative and seem to be in remarkable contrast with the data obtained from animal studies and clinical trials using infliximab. A discrepancy between the effect of etanercept and infliximab has been observed previously in another granulomatous disease, i.e. Crohn's disease [108], and could potentially be explained from differences in the working mechanism of the drugs. Firstly, infliximab binds soluble and membrane-bound TNF $\alpha$ much better than etanercept, resulting in an increased capacity of infliximab to inhibit TNF $\alpha$-mediated cytotoxicity and TNF $\alpha$-induced endothelial cell activation [127]. Secondly, infliximab, but not etanercept, can induce an anti-inflammatory response by reverse signaling through membrane-bound TNF $\alpha$ [128]. Finally, infliximab and etanercept exert different effects on T-lymphocytes [129, 130] and monocytes [131].

\section{Limitations to TNF $\alpha$-inhibiting therapy}

From the studies on TNF $\alpha$-inhibition in ANCA-associated vasculitis, as well as from similar studies performed in patients with other autoimmune diseases, several lessons can be drawn with respect to safety issues regarding this therapy. Generally, TNF $\alpha$-inhibiting therapy can lead to infusion reactions, such as headache, irritation at the site of injection, dizziness, nausea, chest pain, dyspnoea, and pruritus. In addition, treatment with infliximab is associated with a higher rate of infections [91] [119], in particular with an increased risk of reactivation of tuberculosis [132]. In line with these findings, treatment of ANCA-associated vasculitis patients with infliximab resulted in infections in $22 \%$ of the patients [87]. However, in patients with Wegener's 
granulomatosis treated with etanercept [126], as well as in several controlled trials on infliximab in rheumatoid arthritis [90] and ankylosing spondylitis [93], the incidence of infection was similar in the treatment and control group.

The role of TNF $\alpha$ in carcinogenesis is complex, and incompletely understood [133]. The effect of anti-TNF $\alpha$ therapy on the development of malignancies has been predominantly studied in rheumatoid arthritis, a disease that is associated with a higher incidence of malignancies [134]. In this disease, a large case series [135], a controlled retrospective study [136], a large prospective study [134], and a controlled trial [91] suggest that treatment with infliximab or etanercept makes patients more prone to the development or recurrence of cancer, in particular lymphomas. Conversely, Lipsky and colleagues found in a large prospective study that the incidence of malignancies in arthritis patients treated with infliximab was similar to background levels [90]. In the WGET trial, the treatment of Wegener's granulomatosis with etanercept increased the incidence of solid cancer [126], whereas one out of 32 patients with ANCA-associated vasculitis was reported to have developed a B cell lymphoma during treatment with infliximab [87]. Thus, the link between TNF $\alpha$-inhibition and malignancies should be investigated in more detail, before any conclusion can be drawn with respect to this issue.

The high incidence of venous thromboembolic complications entails a new potential threat for patients with Wegener's disease [137]. Bearing this in mind, it is of particular concern to gather information on the thrombogenic effects of TNF $\alpha$-inhibiting therapy. Whereas TNF $\alpha$ is generally thought to be prothrombotic in vivo (reviewed in [138]), the putative anticoagulant effect of TNF $\alpha$-inhibition is challenged by the finding that anti-TNF $\alpha$ can have a prothrombotic effect in chimpanzees [139]. In addition, it is shown that treatment with infliximab or etanercept can induce the formation of potentially prothrombotic anticardiolipin autoantibodies [140]. In the WGET-trial, however, no prothrombotic effect of etanercept was found [126].

Treatment with infliximab as well as etanercept has been associated with increased levels of autoantibodies and/or a lupus-like autoimmune disease (reviewed in [141]). This has been studied most extensively in patients with rheumatoid arthritis [90, 91, 140, 142-145], but anti-TNF $\alpha$ treatment was also shown to induce autoimmunity in ankylosing spondylitis [145], Crohn's disease [121, 122], and mixed connective tissue disease [146]. In the WGET-trial [126], as well as in the infliximab study [87], the incidence of autoimmunity has not been reported. Nevertheless, although the development of autoantibodies in these patients only very rarely leads to a -usually mild- lupus-like syndrome, that resolves after discontinuation of anti-TNF $\alpha$ therapy, treatmentinduced autoimmunity remains an issue of concern. 


\section{Conclusion}

Taken together, in vitro data support the notion that $\mathrm{TNF} \alpha$ plays an important role in the pathogenesis of ANCA-associated vasculitis and NCGN. Consequently, TNF $\alpha$ bioactivityinhibiting therapy might be effective in this disease. This is further supported by several studies in animal models of crescentic GN, although caution should be taken when translating animal studies to the clinical situation. In the only controlled study on TNF $\alpha$ bioinhibition in ANCAassociated vasculitis patients, addition of etanercept to standard immunosuppressive therapy did not lead to improvement, and was associated with the occurrence of serious side-effects. It remains to be seen whether other approaches will be effective. In future studies, patients that are refractory to current treatment strategies should be tested in a controlled fashion, and infliximab should be tested instead of etanercept as additional therapy to standard immunosuppressive regimens. The incidence of potential side-effects, such as infections, malignancies, thromboembolic complications, and autoimmunity, should be of specific concern. 



\section{Chapter 4 - Inhibition of complement factor C5}

protects against anti-myeloperoxidase antibodymediated crescentic glomerulonephritis in mice

Huugen D, van Esch A, Xiao H, Peutz-Kootstra CJ, Buurman WA, Cohen Tervaert JW, Jennette JC, and Heeringa P. Kidney Int 2007; 71: 646-654 


\section{Abstract}

In mice, administration of murine anti-MPO IgG induces pauci-immune necrotizing crescentic glomerulonephritis. Recent studies in this model indicate a crucial role for complement activation in disease induction. Here, we investigated the effect of pretreatment or intervention with a C5-inhibiting monoclonal antibody (BB5.1) in the mouse model of anti-MPO IgG-induced glomerulonephritis.

Mice received BB5.1 8 hours before or 1 day after disease induction with anti-MPO $\operatorname{IgG}$ and LPS. Mice were sacrificed after 1 or 7 days. Control antibody pretreated mice developed hematuria, leukocyturia and albuminuria and glomerulonephritis with a mean of $21.0 \pm 8.8 \%$ glomerular crescents and $12.8 \pm 5.5 \%$ glomerular capillary necrosis. BB5.1 pretreatment prevented disease development, as evidenced by the absence of urinary abnormalities, a marked reduction in glomerular neutrophil influx at day 1 and normal renal morphology at day 7. Importantly, BB5.1 administration 1 day after disease induction also resulted in a marked attenuation of urinary abnormalities and a more than $80 \%$ reduction in glomerular crescent formation.

In conclusion, inhibition of $\mathrm{C} 5$ activation attenuates disease development in a mouse model of anti-MPO IgGinduced glomerulonephritis. These results favor further investigations into the role of complement activation in human MPO-ANCA mediated glomerulonephritis, and indicate that inhibition of C5 activation is a potential therapeutic approach in this disease. 


\section{Introduction}

Wegener's granulomatosis, Churg-Strauss syndrome, microscopic polyangiitis and pauci-immune necrotizing crescentic glomerulonephritis are small vessel vasculitides (SVV) characterized by vascular inflammation predominantly affecting the capillaries, the pre-capillary arterioles and the post-capillary venules [2]. In many cases, renal and pulmonary involvement results in severe renal failure and pulmonary hemorrhage which may be fatal if left untreated. Current therapy consists mainly of immunosuppression with high-dose corticosteroids and cyclophosphamide during the induction phase and azathioprine in combination with low-dose steroids as maintenance therapy. However, considerable treatment-related morbidity and failure to induce or maintain remission underline the importance of less toxic and more effective therapies.

In many cases, SVV are associated with anti-neutrophil cytoplasmic autoantibodies (ANCA) [65]. The main autoantigens in ANCA-associated vasculitis are myeloperoxidase (MPO) and proteinase 3 (PR3), proteins that are predominantly stored in the azurophilic granules of resting neutrophils. In vitro studies suggest a crucial role for MPO-ANCA and PR3-ANCA in the pathogenesis of ANCA-associated SVV (reviewed in [13]) by showing that ANCA are capable of inducing degranulation and a respiratory burst in tumor necrosis factor alpha (TNF $\alpha$-) pretreated neutrophils, resulting in damage of bystander endothelial cells [15]. Definite proof of the pathogenicity of MPO-ANCA came with the development of an experimental animal model of anti-MPO induced necrotizing crescentic glomerulonephritis (NCGN) that involves the transfer of mouse MPO-reactive splenocytes or IgG into MPO-competent mice [43]. These mice developed NCGN with pathological features similar to human anti-MPO-associated glomerulonephritis. Further support for MPO-ANCA pathogenicity is provided by a recently developed rat model, in which immunization of WKY rats with human MPO led to the generation of anti-human MPO antibodies that cross-reacted with rat MPO and caused a mild form of NCGN [49].

In the mouse model of anti-MPO IgG-induced NCGN, we recently showed that circulating neutrophils are crucial in disease development [147], and demonstrated that disease severity was markedly aggravated by the addition of bacterial lipopolysaccharide (LPS) as a proinflammatory stimulus [105]. Moreover, the disease enhancing effects of LPS could be attenuated, but not fully prevented, by pretreatment of the animals with a TNF $\alpha$ bioactivity-inhibiting monoclonal antibody. The partial response on TNF $\alpha$-bioinhibition observed in this study, as well as the ambiguous results obtained from human studies on anti-TNF $\alpha$ therapy in patients with ANCA- 
associated vasculitis $[87,126]$, suggests that other factors are likely to be more critically involved in the induction of anti-MPO mediated NCGN. Recent experiments in mouse strains deficient for various factors of the complement system have indicated a crucial role for the alternative pathway of complement activation in disease induction [148]. More specifically, in these studies it was found that factor B as well as C5 deficient mice are completely protected from anti-MPO antibody induced NCGN, whereas C4 deficient mice are not. These observations prompted us to investigate whether targeting complement factor C5 is a possible therapeutic strategy for intervention. To this end, the present study was designed to examine the effects of a C5inhibiting monoclonal antibody in the mouse model of anti-MPO IgG-induced NCGN.

\section{Methods}

\section{MICE}

$\mathrm{Mpo}^{-/-}$mice, backcrossed to a C57BL/6 background for six times, were genotyped using PCRamplified DNA from tail clippings [61]. Wildtype (WT) female C57Bl/6 (8-12 weeks of age) mice were obtained from Harlan (Horst, the Netherlands). Mice were kept according to University of Maastricht animal facility regulations, and all experiments were approved by the local Animal Care and Experimentation Committee.

\section{PURIFICATION OF MURINE MPO}

Murine MPO (muMPO) was purified from WEHI-3 cells as described previously [105]. Purity was checked by sodium dodecyl sulfate polyacrylamide gel electrophoresis.

\section{Preparation of POLYClONAL MOUSE ANTI-MOUSE MPO IgG}

$\mathrm{Mpo}^{-/-}$mice were immunized with murine MPO as reported previously [105]. On day 42, mice were bled. Anti-MPO antibody titers were monitored by enzyme-linked immunosorbent assay (ELISA) as described previously [105]. IgG was isolated from pooled sera by $50 \%$ ammonium sulfate precipitation followed by protein $G$ column affinity chromatography. IgG-containing fractions were concentrated by ultrafiltration (Centriplus; Millipore, Amsterdam, The Netherlands) and dialyzed against PBS. Protein concentrations were determined using the bicinchoninic acid protein assay kit (Pierce, Rockford, IL). The anti-MPO titer of each batch was checked by ELISA as described above, using a sample of anti-MPO $\operatorname{IgG}$ with previously established pathogenicity as a reference. 


\section{INDUCTION OF GLOMERULONEPHRITIS WITH ANTI-MPO IGG AND LIPOPOLYSACCHARIDE}

C57BL/6 mice received a dose of $100 \mu \mathrm{g} / \mathrm{g}$ body weight of sterile-filtered ( $0.2 \mu \mathrm{m}$ filter; Schleicher \& Schuell, Dassel, Germany) anti-MPO IgG by intraperitoneal injection. Previously, we showed that the administration of LPS considerably aggravated anti-MPO IgG-induced disease resulting in a more vigorous disease model [105]. Therefore, all mice received a single intraperitoneal injection with $0.5 \mu \mathrm{g} / \mathrm{g}$ body weight LPS (Escherichia coli, serotype 026-B6l; Sigma, St. Louis, MO), one hour after the administration of anti-MPO IgG.

\section{ANTI-C5 TREATMENT}

To inhibit C5 function, we used the mouse anti-murine C5 moAb BB5.1. This is a murine IgG1 antibody that blocks C5a-activity as well as the formation of C5b-9 in vivo [149]. ENA-1 (antihuman E-selectin [150]) was used as isotype control.

In a first experiment, C57BL/6 mice were pretreated with an intraperitoneal injection of $1 \mathrm{mg}$ of sterile-filtered BB5.1 ( $\mathrm{n}=9$ ) or isotype control antibody $(\mathrm{n}=9) 8$ hours prior to disease induction with anti-MPO IgG and LPS, to investigate the effect of C5 inhibition in the induction phase of disease. Mice were sacrificed after one day ( $n=4 /$ group) or after 7 days ( $n=5 /$ group) to evaluate disease development.

In the next experiment, we investigated whether intervention with BB5.1 after the induction of disease would lead to attenuation of anti-MPO IgG-induced NCGN. Disease was induced with anti-MPO IgG and LPS as in the first experiment, but this time, $1 \mathrm{mg}$ of BB5.1 or control antibody was given 1 day after disease induction. Mice were sacrificed at 7 days after disease induction ( $\mathrm{n}=5-6 /$ group)

In all mice, circulating anti-MPO IgG was monitored by ELISA as described above, using a serum pool from MPO-immunized MPO-knockout mice as reference.

\section{LABORATORY AND PATHOLOGICAL EVALUATION OF DISEASE}

At several timepoints during disease development, urine samples were tested by dipstick (Bayer, Mijdrecht, the Netherlands) for hematuria and leukocyturia and scored on a 0-4+ scale. At the timepoints indicated, mice were placed in metabolic cages for 16 hours to obtain urine for the determination of albuminuria by ELISA (Bethyl, Inc., Montgomery TX). Blood urea nitrogen (BUN) and creatinin levels were determined in sera collected at the time of sacrifice by an enzymatic degradation assay on a Synchron LX20 PRO (Beckman Coulter Inc., Fullerton CA). 
Tissue samples were taken from both kidneys and processed for light microscopy, immunofluorescence, and immunohistochemistry. For light microscopy, renal tissue samples were fixed in $4 \%$ formaldehyde and embedded in paraffin. Sections $(1.5 \mu \mathrm{m})$ were cut and hematoxylin/eosin, periodic acid-Schiff (PAS), and Martius yellow-brilliant crystal scarlet-soluble blue (MSB) staining were performed according to standard procedures. For each animal, the amount of crescents was determined in 100 consecutive glomerular cross sections. Only glomeruli that had two or more layers of cells in Bowman's space were considered crescentic. Similarly, a glomerular necrosis score was determined for each animal by evaluating segmental or global glomerular capillary necrosis in 100 consecutive glomerular cross sections. Analysis was performed in a blinded fashion by two independent investigators.

Phenotypic analysis of the inflammatory cell infiltrate was performed on $4-\mu \mathrm{m}$ cryostat sections fixed in $100 \%$ acetone at room temperature. The following primary antibodies were used: rat anti-mouse CD45 (clone MP33), rat anti-mouse neutrophils (clone NIMP-R14) [78], rat antimouse CD68 (macrophages, clone FA11 [79]), and rat anti-mouse CD3 (clone KT3). Endogenous peroxidase activity was blocked with $0.05 \% \mathrm{H}_{2} \mathrm{O}_{2}$ in PBS. Rabbit anti-rat IgG-PO and goat anti-rabbit IgG-PO (both DakoCytomation) were used as appropriate secondary and tertiary antibodies, respectively. Antibody binding was visualized using 3-amino-9-ethylcarbazole (AEC) with $\mathrm{H}_{2} \mathrm{O}_{2}$ as substrate. Sections were counterstained with hematoxylin. Glomerular cell infiltrates were determined by counting the number of positive cells in 30 glomerular cross sections per kidney section.

The presence of complement proteins was determined by immunofluorescence using the following primary antibodies: polyclonal digoxigenin- (dig-) labeled rabbit-anti-mouse C1q (generously provided by Prof. dr. MR Daha, Leiden, the Netherlands [151]), monoclonal rat-antimouse C4 (clone 16D2; HBT, Uden, the Netherlands), monoclonal rat-anti-mouse C3 (clone 11H9; HBT), and rabbit anti-mouse C5 (HBT) . FITC-labeled sheep-anti-dig IgG (Roche Diagnostics, Almere, the Netherlands), ALEXA 568-labeled goat-anti-rat IgG, and ALEXA 568labeled goat-anti-rabbit (Invitrogen, Breda, The Netherlands) supplemented with 5\% normal sheep serum were used as conjugates. Deposition of mouse IgG was detected by rabbit antimouse IgG-Alexa Fluor 488 (Invitrogen).

\section{STATISTICAL ANALYSIS}

Data are expressed as mean $\pm \mathrm{SD}$ and were analyzed by two-tailed Student's t-test using Graphpad Prism 4.03 for Windows (Graphpad Software, San Diego CA). Because of their non- 
Gaussian distribution, albuminuria data were compared by the two-tailed Mann-Whitney $\mathrm{U}$ test. In both cases, $\mathrm{p}$-values $\leq 0.05$ were considered statistically significant.

\section{Results}

\section{INHIBITION OF C5 ACTIVATION PREVENTS NECROTIZING CRESCENTIC GLOMERULONEPHRITIS INDUCED BY ANTI-MPO IGG AND LPS}

\section{Urinalysis}

Upon administration of anti-MPO IgG and $0.5 \mu \mathrm{g} / \mathrm{g}$ LPS, mice in both groups developed similar levels of circulating MPO-ANCA (data not shown). Control-pretreated mice developed hematuria and leukocyturia that lasted from day one until sacrifice on day 7 (Figure 4-1a, b). Urinary albumin concentrations were within normal range on day 1 post-induction (mean 48 (range, 10-145) $\mu \mathrm{g} / 16 \mathrm{~h}$ ), but were markedly increased on day 7 (mean 1600 (range, 430-3300) $\mu \mathrm{g} / 16 \mathrm{~h}$, Figure 4-1c). In contrast, urine samples of mice pretreated with anti-C5 antibody displayed only minimal amounts of blood and leukocytes throughout the experiment (Figure 41a, b), and albuminuria was within normal range. (day 1: mean 45 (range, 6-100) and day 7: mean 23 (range, 14-42) $\mu \mathrm{g} / 16 \mathrm{~h}$; Figure 4-1c). At sacrifice, creatinine and blood urea nitrogen levels were within normal range in all mice.
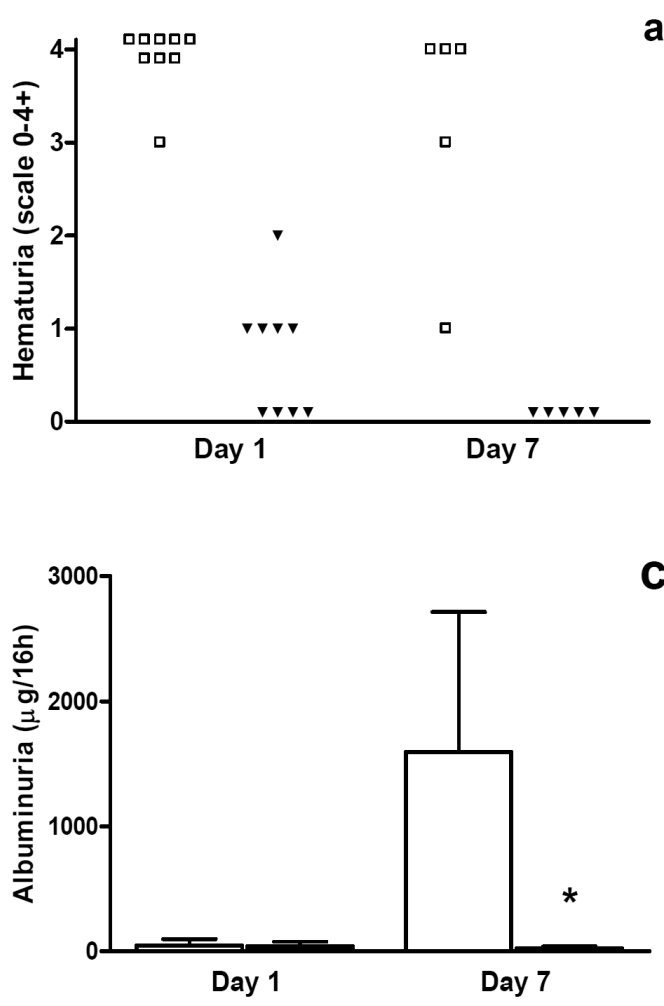

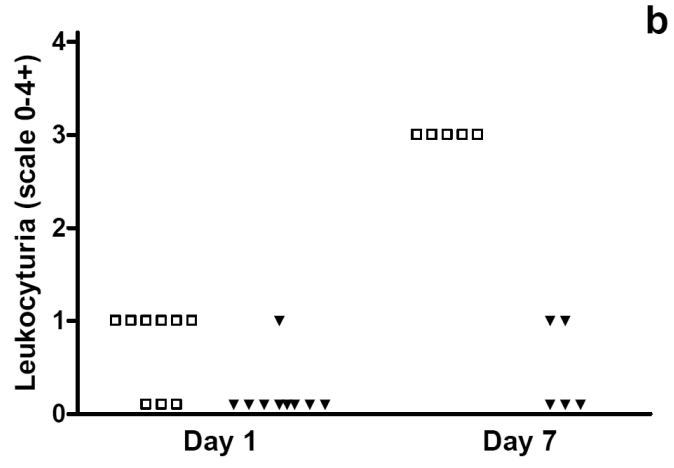

C Figure 4-1: Pretreatment with anti-C5 moAb inhibits induction of (a) hematuria, (b) leukocyturia, and (c) albuminuria induced by anti-MPO IgG and LPS. Open squares: control pretreatment; filled triangles: anti-C5 pretreatment. The mean albumin level in urine samples from untreated C57bl/6 $(n=5)$ mice was 43 (range 26-79) $\mu \mathrm{g} / 16$ hours. $* \mathrm{p}<0.05$. 


\section{Light microscopy}

In mice pretreated with control antibody, the administration of anti-MPO IgG and LPS induced a focal and segmental crescentic glomerulonephritis on day 7 in all subjected mice. Although the majority of glomeruli appeared unaffected, glomerular crescent formation and necrosis were frequently observed. The mean percentage of crescentic glomeruli was $21.0 \pm 8.8$, whereas $12.8 \pm$ $5.5 \%$ of glomeruli showed fibrinoid necrosis (Figure 4-2). Crescents were cellular, and they were partly segmental $(9 \%$ of the total number of glomeruli) and partly global ( $13 \%$ of total). Accompanying the crescentic glomerulonephritis, there was mild tubulo-interstitial inflammation, which in some cases was accompanied by mild tubular atrophy. Arterioles and larger blood vessels were unaffected.
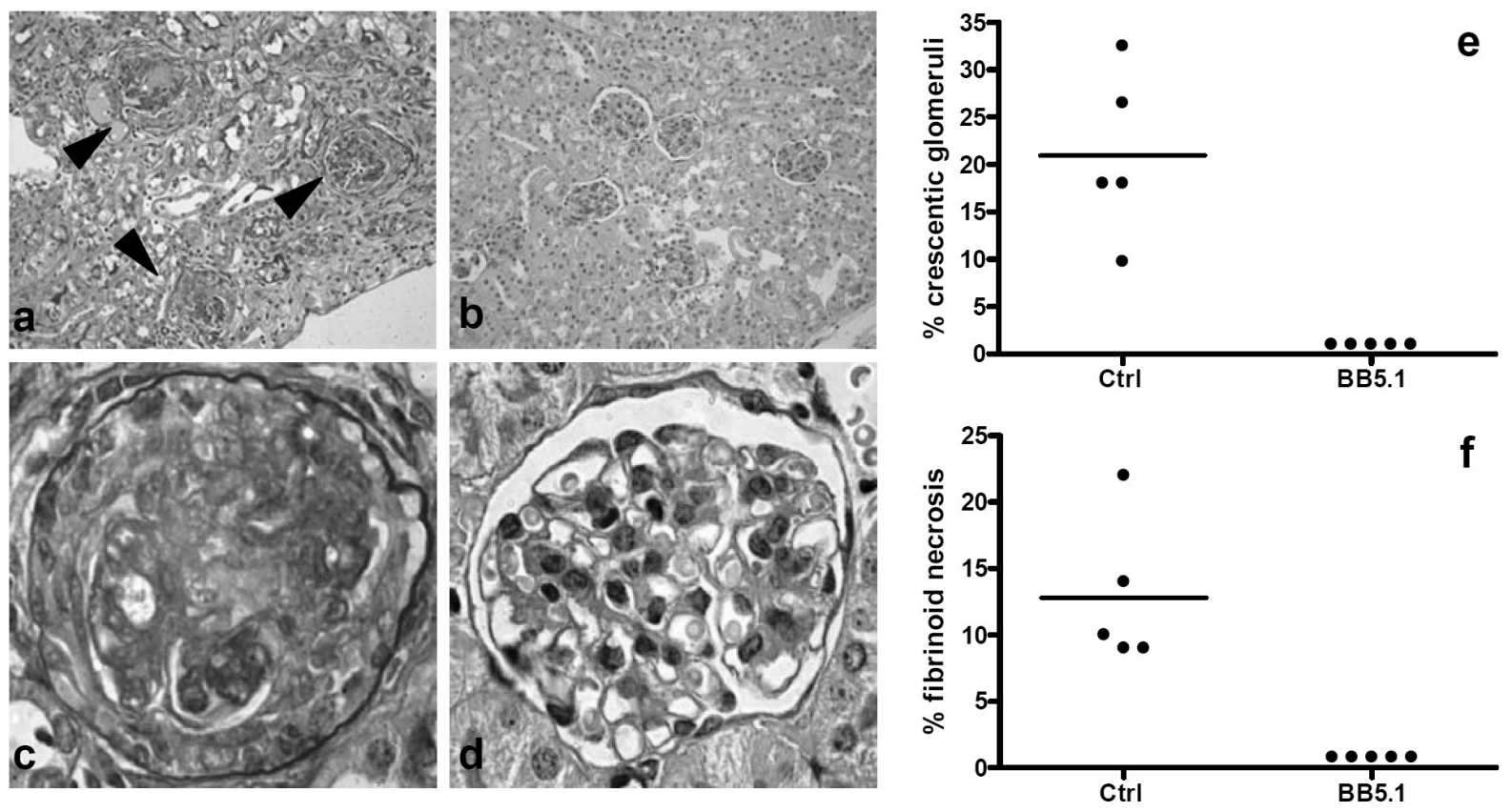

Figure 4-2: Pretreatment with anti-C5 moAb prevents development of NCGN induced by anti-MPO IgG and LPS. a: Overview of renal cortical tissue from a control antibody pretreated mouse, 7 days after administration of antiMPO IgG and LPS, representing the focal and segmental nature of the glomerulonephritis. Glomerular crescents are indicated by arrowheads. b: Overview of renal cortical tissue from an anti-C5 moAb treated mouse, 7 days after administration of anti-MPO IgG and LPS, displaying normal renal morphology. c: Detail of a glomerulus with a large cellular crescent from a mouse that received anti-MPO IgG and LPS after pretreatment with control antibody. d: Detail of a glomerulus from a mouse that received anti-MPO IgG and LPS after pretreatment with anti-C5 moAb, displaying normal morphology e: Effect of pretreatment with anti-C5 moAb on glomerular crescent formation expressed as a percentage of glomerular crescents in individual mice. f: Effect of pretreatment with anti-C5 moAb on glomerular necrosis expressed as a percentage of glomeruli with capillary necrosis in individual mice. Horizontal lines represent mean percentages in each group. a-d Periodic acid Schiff's stain. Magnification: a,b x200; d,e x630. 
In contrast, glomerular crescents and fibrinoid necrosis were completely absent in mice pretreated with C5-inhibiting antibody (Figure 4-2). In addition, no tubulo-interstitial inflammation or tubular atrophy could be found in these mice. Phenotypic analysis of the inflammatory cell infiltrate on day 7 revealed that anti-C5 pretreatment significantly inhibited glomerular influx of $\mathrm{CD}_{4} 5^{+}$leukocytes, $\mathrm{CD}^{+}$T-cells, FA11 ${ }^{+}$macrophages, and NimpR14 neutrophils (Table 4-1).

Table 4-1: Immunophenotyping of intraglomerular inflammatory cell infiltrate at 7 days after pretreatment with antiC5 moAb (BB5.1) or isotype control moAb (ENA-1).

\begin{tabular}{|c|c|c|c|}
\hline & $\begin{array}{l}\text { Control-pretreatment* } \\
\qquad(\mathrm{n}=5)\end{array}$ & $\begin{array}{l}\text { Anti-C5 pretreatment* } \\
\qquad(\mathrm{n}=5)\end{array}$ & p-value \\
\hline $\mathrm{CD} 5^{+}$leukocytes & $3.2 \pm 0.59$ & $1.2 \pm 0.21$ & 0.0004 \\
\hline $\mathrm{CD}^{+}{ }^{+} \mathrm{T}$-cells & $0.45 \pm 0.11$ & $0.13 \pm 0.05$ & 0.0011 \\
\hline $\mathrm{FA}_{11^{+}}$macrophages & $2.3 \pm 0.62$ & $0.68 \pm 0.32$ & 0.002 \\
\hline NIMP-R14 $^{+}$neutrophils & $0.41 \pm 0.12$ & $0.22 \pm 0.09$ & 0.03 \\
\hline
\end{tabular}

${ }^{*}$ Number of cells (mean $\pm \mathrm{SD}$ ) per glomerular cross-section

To gain more insight into the mechanism by which anti-C5 antibodies affect disease development in the mouse model of anti-MPO IgG-induced NCGN, we investigated the effect of anti-C5 pretreatment on early glomerular neutrophil influx. Compared to the control antibody, anti-C5 antibody pretreatment significantly reduced the number of neutrophils per glomerular crosssection in mice one day after disease induction with anti-MPO IgG and LPS ( $2.5 \pm 0.66$ vs. 0.79 \pm 0.27 /gcs, $\mathrm{p}<0.005$; Figure 4-3).
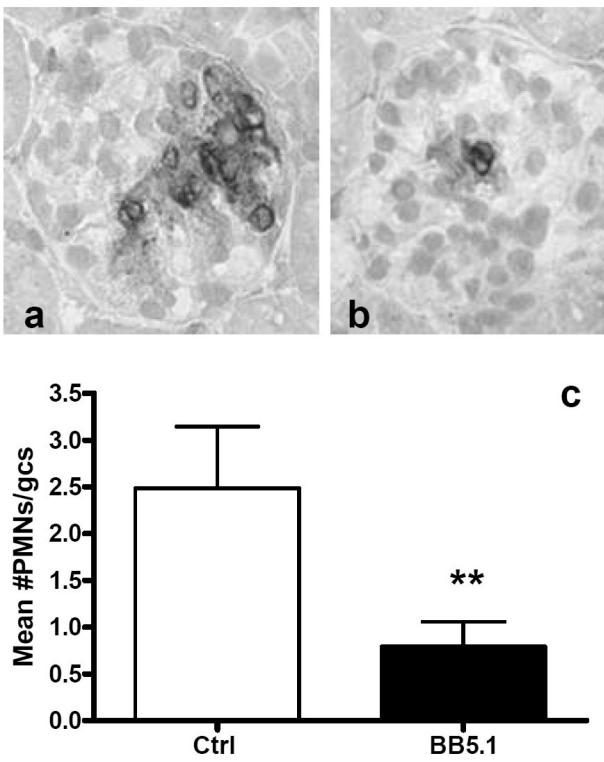

Figure 4-3: Pretreatment with anti-C5 moAb significantly reduces early glomerular neutrophil influx. a: Glomerulus from a mouse that had received anti-MPO $\operatorname{IgG}$ and LPS and was pretreated with control antibody demonstrating marked segmental infiltration of neutrophils as detected by immunohistochemistry. b: Glomerulus from a mouse that had received anti-MPO IgG and LPS and was pretreated with antiC5 moAb, demonstrating strongly reduced neutrophil infiltration. c: Quantification of glomerular neutrophil influx one day of administration of anti-MPO IgG and LPS in mice that were pretreated with control antibody (white bar) or antiC5 moAb (black bars). gcs, glomerular cross section; magnification a,b x630. $* * \mathrm{p}<0.01$. 


\section{Immunofluorescence}

Immunofluorescence staining for complement factors C1q, C3, and C4 in anti-MPO/LPS treated and untreated wildtype mice sacrificed at day one showed sparse glomerular staining for C1q and weak mesangial staining for C3 in all mice (Figure 4-4). Also, mesangial C4 deposits were observed. The intensity and pattern of C1q, C3, and C4 staining was similar to that observed in untreated C57Bl/ 6 mice (Figure 4-4). In contrast, glomerular capillary staining for C1q, C3, and C4 was detected in renal sections from mice one day after induction of heterologous anti-GBM glomerulonephritis (Figure 4-4). In these mice, staining for C5 demonstrated moderate granular glomerular deposits. However, such deposits were not detected in untreated C57Bl/6 mice and anti-MPO/LPS treated mice. Immunofluorescence staining for IgG revealed only scant deposits of $\mathrm{IgG}$ in glomeruli in all mice (data not shown).

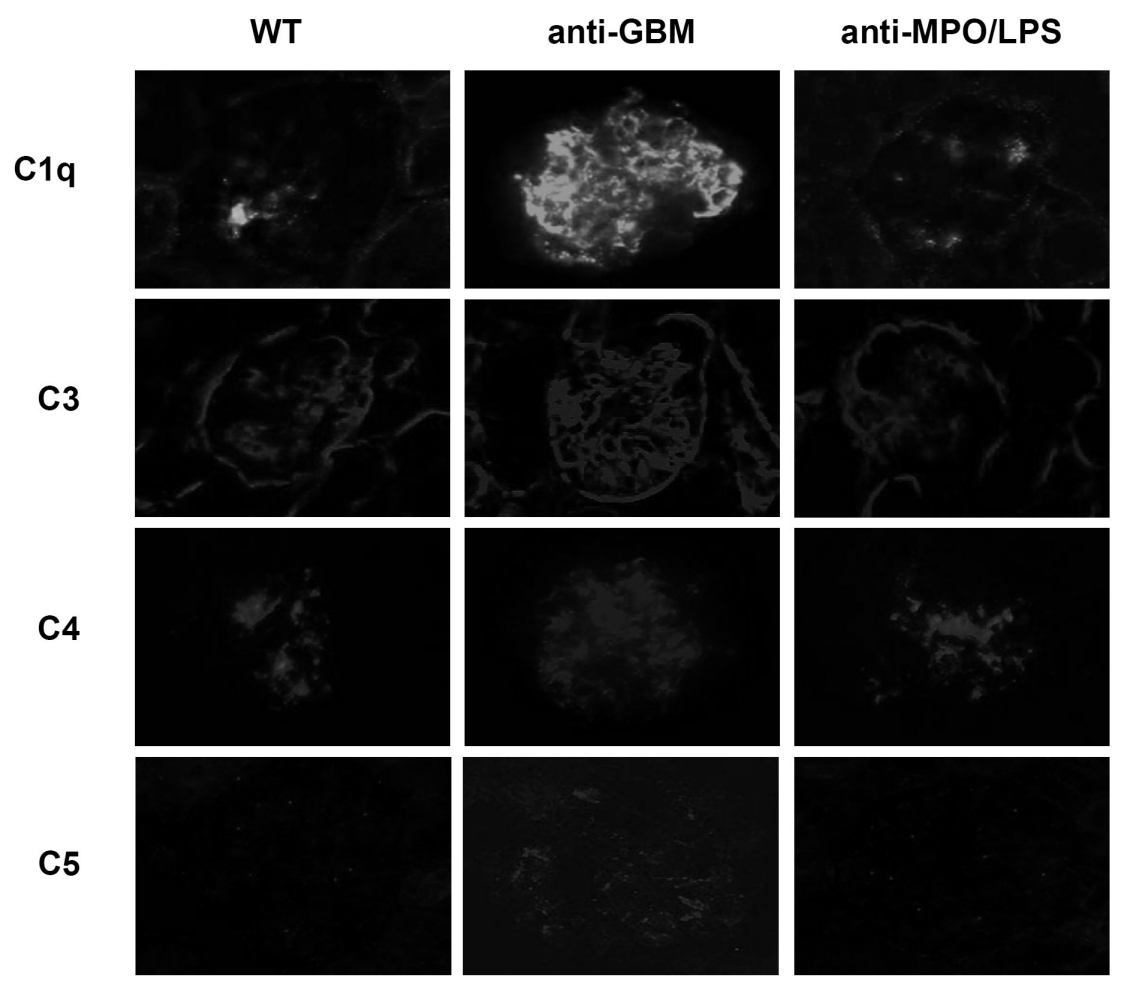

Figure 4-4: Immunofluorescence staining for complement proteins in untreated wildtype C57bl/6 mice and in mice one day after injection with anti-MPO IgG and LPS or heterologous rabbit anti-glomerular basement membrane (GBM) antibodies. Representative glomeruli stained for C1q, C3, C4, and C5 are shown. In wildtype mice and mice sacrificed one day after injection of anti-MPO IgG and LPS sparse glomerular staining for C1q, weak mesangial staining for $\mathrm{C} 3$ and mesangial deposits of $\mathrm{C} 4$ were observed. In contrast, in mice sacrificed one day after injection of rabbit anti-GBM antibodies, capillary staining for C1q, C3 and, C4 was found. For C5, moderate granular glomerular deposits were observed in renal sections of mice subjected to accelerated sheep anti-GBM nephritis whereas no glomerular staining was detected in untreated C57Bl/6 mice and anti-MPO/LPS treated mice. Magnification all pictures $\times 400$. 


\section{INHIBITION OF C5 ACTIVATION AFTER DISEASE INDUCTION ATTENUATES NECROTIZING CRESCENTIC GLOMERULONEPHRITIS INDUCED BY ANTI-MPO IGG AND LPS}

\section{Urinalysis}

We next investigated whether anti C5-treatment was also beneficial when administered one day after the induction of disease with anti-MPO IgG and LPS. We previously demonstrated that at this timepoint, considerable glomerular neutrophil influx is already present [105]. Moreover, at the start of intervention, mice in both groups had similar levels of circulating anti-MPO antibodies and had developed hematuria and leukocyturia to a similar extent (Figure 4-5a, b). As shown in Figure 4-5a, the extent of hematuria declined in mice treated with a single dose of BB5.1, and was absent on day five. At the time of sacrifice on day 7, three out of six BB5.1treated animals were still free from hematuria, whereas hematuria persisted in control-treated animals.


Figure 4-5: Intervention with anti-C5 moAb attenuates (a) hematuria, (b) leukocyturia and (c) albuminuria induced by anti-MPO IgG and LPS. Open squares: control treatment; Filled triangles: anti-C5 treatment. $* * \mathrm{p}<0.01$. 
Anti-C5 treatment also had a pronounced effect on leukocyturia. In control antibody-treated mice, leukocyturia increased during the time course of the experiment, whereas this increase was absent in mice treated with anti-C5 antibody (Figure 4-5b). In both groups, albuminuria was quantified in urine samples obtained on day 1 and day 7 post-induction. As shown in Figure 4-5c, urinary albumin levels on day 1 were within normal range in both groups. On day 7 however, a significant increase in albuminuria was detected in control antibody-treated mice, whereas in antiC5 antibody-treated mice urinary albumin levels were only minimally elevated (mean 212 (range, 45-633) $\mu \mathrm{g} / 16 \mathrm{~h}$ in anti-C5 antibody-treated mice vs. 1596 (range, 705-2552) $\mu \mathrm{g} / 16 \mathrm{~h}$ in control antibody-treated mice, $\mathrm{p}<0.01)$.

\section{Light microscopy}

On day 7 , all control antibody-treated mice had developed a focal and segmental NCGN, with a mean of $13.9 \pm 4.5 \%$ crescentic glomeruli, and $11.8 \pm 3.4 \%$ of glomeruli displaying capillary fibrinoid necrosis. The development of NCGN was however markedly attenuated in the anti-C5 antibody-treated mice, showing a mean of $1.9 \pm 0.8 \%$ glomerular crescents, and $1.5 \pm 1.2 \%$ of the glomeruli containing capillary fibrinoid necrosis (Figure 4-6).

Also, treatment with the C5-inhibiting antibody led to a significant reduction in glomerular influx of $\mathrm{CD}_{4} 5^{+}$leukocytes as well as $\mathrm{CD}^{+}{ }^{+}$-cells and $\mathrm{FA} 11^{+}$macrophages on day 7 (Table 4-2). In both groups, glomerular influx of NIMP-R14 ${ }^{+}$neutrophils on day 7 was low and the difference between the groups did not reach statistical significance. (Table 4-2) Staining for complement proteins in these groups of mice were similar to those of the pretreatment study. There were no differences in C1q, C3, C4, and C5 staining between BB5.1-treated and control-treated mice, and the intensity and pattern in both groups were similar to the untreated wildtype C57bl/6 mice (data not shown). 



Figure 4-6: Intervention with anti-C5 moAb attenuates development of NCGN induced by anti-MPO antibodies and LPS. a: Overview of renal cortical tissue from a control antibody treated mouse 7 days after administration of antiMPO IgG and LPS representing the focal and segmental nature of the induced glomerulonephritis . Glomerular crescents are indicated by arrowheads. b: Overview of renal cortical tissue from an anti-C5 moAb treated mouse 7 days after administration of anti-MPO IgG and LPS . One glomerular crescent is observed (arrowhead). c: Detail of a glomerulus with a cellular crescent from a mouse that received anti-MPO IgG and LPS and was treated with control antibody. $\mathbf{d}$ : Detail of a glomerulus with a small cellular crescent from a mouse that received anti-MPO IgG and LPS and was treated with anti-C5 moAb. e: Effect of treatment with anti-C5 moAb on glomerular crescent formation expressed as a percentage of glomerular crescents in individual mice. $\mathbf{f}$ : Effect of treatment with anti-C5 moAb on glomerular necrosis expressed as a percentage of glomeruli with capillary necrosis in individual mice. Horizontal lines represent mean percentages in each group. a-d Periodic acid Schiff's stain. Magnification: a,b x200; d,e $x 630 .{ }^{* * *} \mathrm{p}<0.001$.

Table 4-2: Immunophenotyping of intraglomerular inflammatory cell infiltrate at 7 days after treatment with anti-C5 moAb (BB5.1) or isotype control moAb (ENA-1).

\begin{tabular}{lccc}
\hline & $\begin{array}{c}\text { Control-treatment } \\
(\mathbf{n}=\mathbf{5})\end{array}$ & $\begin{array}{c}\text { Anti-C5 } \\
(\mathbf{n}=\mathbf{6})\end{array}$ & p-value \\
\hline $\mathrm{CD}^{*} 5^{+}$leukocytes & $3.2 \pm 0.76$ & $1.7 \pm 0.19$ & 0.0011 \\
$\mathrm{CD}^{+}$T-cells & $0.51 \pm 0.20$ & $0.14 \pm 0.06$ & 0.0017 \\
$\mathrm{FA}^{+} 1^{+}$macrophages & $1.85 \pm 0.30$ & $0.9 \pm 0.18$ & 0.0001 \\
NIMP-R14 $^{+}$neutrophils & $0.37 \pm 0.09$ & $0.27 \pm 0.08$ & n.s. \\
\hline
\end{tabular}

${ }^{*}$ Number of cells (mean $\pm \mathrm{SD}$ ) per glomerular cross-section; n.s.: not significant 


\section{Discussion}

In the present study, we demonstrate that mice pretreated with BB5.1, a moAb that inhibits activation of complement factor C5, are completely protected from disease induced by anti-MPO IgG and $0.5 \mu \mathrm{g} / \mathrm{g}$ bacterial LPS. Control-pretreated mice developed focal segmental NCGN to a degree comparable with our previous observations [105]. Importantly, we also demonstrate that intervention with BB5.1 after the onset of disease, at a time that important signs of disease, such as hematuria, leukocyturia and glomerular influx of neutrophils, are already present, was also effective, as evidenced by a more than $80 \%$ reduction in the percentage of crescentic glomeruli and a more than $85 \%$ reduction in albuminuria on day 7 .

Our study is the first to show that C5-inhibition prevents disease development in an animal model of ANCA mediated NCGN. These results are in line with two other studies on immunemediated glomerular injury showing involvement of the terminal complement pathway in a mouse model of lupus nephritis [152] and glomerulonephritis induced by cryoglobulins [153]. According to the currently prevailing theory on the pathogenesis of ANCA mediated vasculitis (reviewed in [18]), ANCA-induced activation of primed neutrophils is crucial to disease development. ANCA-activated neutrophils adhere to susceptible vascular endothelium, where they release oxygen radicals and lytic granule constituents, leading to local inflammation. Although this theory has proven very useful, it does not explain the critical involvement of complement in ANCA-induced disease.

Complement system activation can occur through the classical pathway, which is dependent on immune complexes (ICs), through the mannose-binding lectin (MBL) pathway, or through alternative pathway activation. Because of the paucity of ICs in renal biopsies from patients with ANCA-associated NCGN, it seems unlikely that the classical pathway plays an important pathogenic role. Immune deposits have however been found in fresh skin lesions from WG patients, suggesting their involvement in the initial phase of disease development [62]. Moreover, IC depositions were found in over 50\% of renal biopsies from ANCA-associated vasculitis patients [154] and their presence was associated with higher levels of proteinuria. This association does however not reveal whether IC positivity is the cause of more severe disease manifestations, or whether it is merely the result of more severe disease. Finally, we previously reported that levels of circulating MPO in mice are increased upon treatment with LPS which could potentially lead to the formation of circulating MPO/anti-MPO immune complexes in our model. (8) However, whether circulating immune complexes are formed and whether these participate in disease induction is unknown at present and warrants further investigation. In this respect, it is 
important to note that C4-deficient mice which are unable to activate the classical complement pathway, are not protected from disease induced by anti-MPO IgG [148]. Moreover, in our present study, glomerular depositions of $\operatorname{IgG}$ and $\mathrm{C} 1 \mathrm{q}$ were scanty and not different from those observed in untreated mice. Together, these results suggest that disease development in the mouse model of anti-MPO IgG-induced NCGN is not dependent on activation of the classical pathway of complement activation.

Involvement of the MBL pathway of complement activation has been demonstrated in several forms of glomerulonephritis, including IgA nephropathy and lupus nephritis [155]. However, whether MBL pathway activation contributes to ANCA mediated SVV is largely unknown and has not been thoroughly investigated. A recent study showed that in patients with ANCAassociated vasculitis, genotype frequencies of polymorphic variants in the MBL gene are not different from those observed in the normal population (Kamesh L, Heward JM, Williams JM, et al. Kidney Blood Press Res 28:176, 2005, abstract). Moreover, mice deficient for C4, one of the key components of the MBL cascade, are not protected from disease induction in the model of anti-MPO IgG-induced NCGN [148]. Together, these data indicate that involvement of the MBL pathway in ANCA mediated SVV is unlikely.

The initiating event in the alternative pathway of the complement cascade is the spontaneous generation of the $\mathrm{C} 3$ convertase, $\mathrm{C} 3 \mathrm{bBb}$. This complex is however very unstable, and its proper functioning is therefore dependent on the stabilizing effect of the binding of properdin. Interestingly, it has been shown that properdin is one of the proteins that is released by activated neutrophils [156]. Stabilizing the C3 convertase and consequent potentiation of the complement cascade might therefore be an important additional deleterious effect of neutrophil activation in ANCA-associated vasculitis. However, very little is known about the C3-binding and activating properties of ANCA. In one study, ANCA-positive sera were found to facilitate the binding of complement factor C3c, as well as the formation of C5b-9, on neutrophils [157]. Bearing in mind the results of our study, this potential mechanism of direct ANCA-induced complement activation certainly merits further investigations.

The enzymatic activity of MPO and other neutrophil lysosomal constituents may provide an alternative -or additional- link between MPO-ANCA and complement activation. It has been shown that C5 can acquire neutrophil chemotactic activity upon treatment with an enzyme stored in the lysosomal granules of granulocytes, but the nature of this enzyme remains unclear [158]. Interestingly, C5 acquires C5b-like activity upon treatment with hypochlorite and hypochloritemodified amines, either from an exogenous source [26], or as the enzymatic product of MPO 
released from activated neutrophils [27]. Since the majority of MPO-ANCA do not inhibit MPOactivity, but actually prevent the inhibition of MPO-activity by its natural inhibitor, ceruloplasmin [29], it is conceivable that release of MPO, in the presence of MPO-ANCA, leads to MPOinduced activation of the terminal complement pathway. It is however unclear in these studies whether, during the formation of the C5b-like complex, also a protein with C5a-like properties is formed. In addition, this mechanism does not provide an explanation for the critical role of alternative pathway activation in anti-MPO IgG-induced NCGN [148]

Upon activation, C5 is spliced into two cleavage products. The C5b molecule is involved in the generation of C5b-9, the end product of the terminal complement pathway. The C5a molecule is a powerful chemoattractant for neutrophils, and has considerable neutrophil activating potential. Since BB5.1 inhibits C5a-activity as well as the formation of C5b-9 [149], its effect on anti-MPO IgG-induced NCGN can theoretically be caused by the inhibition of C5a activity, inhibition of C5b-9 formation, or both.

Very little is known about the role of C5b-9 in ANCA-associated glomerulonephritis. On neutrophil smears, it has been demonstrated that ANCA-positive sera are capable of binding C3 which, in some cases, resulted in the formation of C5b-9 [157]. It is however unclear whether this mechanism also plays a role in vivo. Alternatively, C5a activity could play a critical role in the pathogenesis of MPO-ANCA associated vasculitis. C5a has strong neutrophil-attracting capability, and we previously showed that, in the mouse model of anti-MPO IgG-induced NCGN, neutrophils are crucial for disease development [147]. In our current study, we observed that anti-C5 pretreatment led to a significant decrease in early glomerular neutrophil influx on day one. This is in line with several studies showing that C5a strongly promotes the adhesion of neutrophils to endothelial cells $[159,160]$. Being a strong neutrophil activator, C5a might induce the adherent neutrophils to subsequently undergo an oxidative burst and release their granular enzymes. Interestingly, C5a is also a strong inducer of properdin release by neutrophils [156], and might thus be part of a positive feedback loop stabilizing the alternative pathway convertase, thereby increasing inflammation [161]

It has previously already been shown by our group that neutrophils play a pivotal role in the pathogenesis of anti-MPO IgG-induced NCGN [147]. However, whereas macrophages importantly contribute to glomerular lesions in this, as well as in a previous [105] study, it is as yet unclear as to what extent these cells are involved in the pathogenic process. It has been shown that monocytes, being the circulating precursors of macrophages, are activated in humans with active ANCA-associated disease [162], and in vitro studies have revealed that ANCA themselves 
might be at least partly responsible for this [163]. Moreover, macrophages have frequently been shown to be the predominant cell type in glomerular lesions of patients with ANCA-associated vasculitis $[164,165]$ (reviewed in [166]). These studies do however not unequivocally prove a role for monocytes and macrophages in ANCA-disease. Therefore, although in our study significantly less glomerular macrophages are observed in experimental mice pretreated with anti-C5, and although the activating effect of $\mathrm{C} 5 \mathrm{a}$ on monocytes and macrophages is well-known, additional studies are required to reveal whether the observed reduction in macrophage influx is pathogenetically involved in the effect of anti-C5 treatment in this disease model.

Murine anti-MPO IgG induced NCGN is in many ways similar to MPO-ANCA associated NCGN observed in humans. As in human disease, immune complexes are scarce or absent in the mouse model [43, 105], and murine anti-muMPO IgG activates TNF $\alpha$-primed murine neutrophils [105]. The results of the present study together with the observations that murine anti-MPO induced NCGN is dependent on an intact alternative pathway complement system indicate a previously unsuspected role for complement activation in ANCA associated diseases. Clearly, these results require confirmation and translation to the human situation.

\section{Acknowledgments}

We would like to thank Maria Vroomen (University Maastricht; Internal Medicine, Division of Clinical and Experimental Immunology) for her assistance in the immunohistochemical analysis of this study. We thank Prof. M.R. Daha (dept. of Nephrology, Leiden University Medical Center, the Netherlands) and dr S. Asgeirsdottir (dept. of Medical Biology, University Medical Center, Groningen, the Netherlands) for providing renal sections from heterologous anti-GBM treated mice. 

Chapter 5 - Monoclonal antibodies specific for murine myeloperoxidase aggravate mild antiglomerular basement membrane (GBM) antibodyinduced glomerulonephritis

Huugen D, van Esch A, Cohen Tervaert JW, and Heeringa P. Manuscript in preparation 


\section{Abstract}

Anti-neutrophil cytoplasmic autoantibodies specific for myeloperoxidase (MPO) are associated with small-vessel vasculitis in humans. The administration of polyclonal IgG, isolated from murine MPO-immunized MPO-deficient $\left(M p o^{-/}\right)$mice, causes vasculitis in wildtype (WT) mice. Using splenocytes from murine MPO-immunized $M p o^{-/-}$mice, we generated and characterized a panel of 11 mouse anti-mouse MPO monoclonal antibodies (moAbs). Whereas most of them cross-react with rat MPO, only one moAb shows weak cross-reactivity with human MPO. Inhibition ELISA revealed that their epitopes are restricted to three immunodominant regions on the murine MPO molecule. In several combinations, alone or with a priming dose of bacterial lipopolysaccharide (LPS), the anti-MPO moAbs do not cause glomerulonephritis in WT mice. However, two clones severely aggravated mild crescentic glomerulonephritis induced by the administration of a low dose of anti-glomerular basement membrane (GBM) IgG, as reflected by increased hematuria, albuminuria, and histopathological damage, and more severely impaired renal function. Together, these results underline the pathogenicity of the anti-MPO immune response, and provide an interesting tool to study the role of epitope specificity and isotype in the pathogenesis of anti-MPO IgG-induced NCGN. 


\section{Introduction}

Small vessel vasculitides (SVV) are diseases that are characterized by destructive inflammation of the arterioles, capillaries and postcapillary venules [2]. Although any organ can be affected, renal and pulmonary involvement typically lead to severe morbidity, and may be fatal if left untreated. In many patients, the presence of SVV is associated with elevated titers of anti-neutrophil cytoplasmic autoantibodies (ANCA). The autoantibodies are predominantly specific for myeloperoxidase (MPO-ANCA) or proteinase 3 (PR3-ANCA), proteins that are stored in the lysosomal granules of polymorphonuclear granulocytes (PMNs) and monocytes, and that have an important bactericidal function [20]. Since the discovery of ANCA, several studies have shown that ANCA-titers correlate with disease activity and predict relapse and therefore, ANCA detection is considered to be an important tool in the diagnosis and follow-up of patients with ANCA-associated SVV [7, 66-68, 167].

Since the discovery of ANCA, their role in the pathogenesis of ANCA-associated disease has been subject of investigation. It has been shown in vitro that ANCA can activate TNF $\alpha$-primed PMNs, and this has led to a theory on the pathogenesis of ANCA-associated SVV (reviewed in [18]), in which ANCA-activated neutrophils adhere to and damage endothelium of susceptible blood vessels, leading to local vascular inflammation. Although many other animal studies (reviewed in [168]) already strongly supported this notion, definite evidence for pathogenicity of MPO-ANCA was provided by Xiao et al., showing that the adoptive transfer of splenocytes or the passive transfer of purified $\mathrm{IgG}$ from murine MPO-immunized MPO-deficient $\left(\mathrm{Mpo}^{-/}\right)$mice to immune-deficient or wildtype (WT) mice resulted in pauci-immune necrotizing crescentic glomerulonephritis that was similar to human MPO-ANCA associated glomerulonephritis [43]. Since then, it has been shown that neutrophils play a key role in the passive transfer model of anti-MPO IgG-induced NCGN [147], and that proinflammatory stimuli aggravate disease [105]. In the mouse model of anti-MPO IgG-induced NCGN, disease is induced by the passive transfer of polyclonal anti-MPO $\operatorname{IgG}$, obtained from sera of MPO-immunized $M p 0^{-/}$mice. It is conceivable that in this mixture of antibodies with different epitope specificities and isotypes, only a subpopulation contributes to its pathogenic effect. In this study, we describe the development and characterization of a panel of mouse anti-MPO monoclonal antibodies (moAbs) that can be used to study the role of idiotype and isotype in murine anti-MPO IgGinduced NCGN. In addition, we show that two of those moAbs are pathogenic in the context of a mild local proinflammatory environment induced by the administration of a low dose of anti- 
glomerular basement membrane (GBM) antibodies. Our study confirms the pathogenicity of the anti-MPO immune response, and provides an interesting tool to study the involvement of epitope restriction and isotype in the pathogenesis of anti-MPO IgG-induced NCGN.

\section{Material and Methods}

\section{MICE}

$\mathrm{Mpo}^{-/-}$mice, backcrossed to a $\mathrm{C} 57 \mathrm{Bl} / 6$ background for six times, were genotyped using PCRamplified DNA from tail clippings [61]. Wildtype female C57Bl/6 (8-12 weeks of age) mice were obtained from Harlan (Horst, the Netherlands). Mice were kept according to University of Maastricht animal facility regulations, and all experiments were approved by the local Animal Care and Experimentation Committee.

\section{GENERATION AND CHARACTERIZATION OF ANTI-MPO MOABS}

\section{Immunization and generation of hybridomas}

To obtain anti-MPO IgG-producing hybridomas, $\mathrm{Mpo}^{-/-}$mice were immunized with MPO isolated from the murine promyelocytic cell line WEHI-3 as described previously [105]. Mice received an intraperitoneal dose of $10 \mu \mathrm{g}$ murine MPO in $100 \mu \mathrm{l}$ sterile-filtered PBS and an equal volume of Complete Freund's Adjuvant (Sigma, Zwijndrecht, The Netherlands) on day 0, followed by two booster injections of $10 \mu \mathrm{g}$ murine MPO in Incomplete Freund's Adjuvant (Sigma) on day 21 and 35. on day 43, $10 \mu \mathrm{g}$ of murine MPO in PBS was administered, and at day 48, the animals were sacrificed, the spleens were harvested, and a single-cell suspension was made by pressing the cells through a sterile nylon gaze. Subsequently, cells were resuspended in RPMI Medium 1640 with GlutaMAX ${ }^{\text {TM}_{-}}$(Invitrogen, Breda, The Netherlands), enriched with 20\% fetal calf serum, 1\% penicillin/streptamycin (Gibco), HAT (0.1 mM hypoxanthine, $0.4 \mu \mathrm{M}$ aminopterin, $16 \mu \mathrm{M}$ thymidine; Invitrogen), and $0.1 \% \beta$-mercaptoethanol, and fused with the HAT-sensitive cell line $\mathrm{SP}_{2} 0$ by co-culturing them in the presence of polyethylene glycol (Serva, Heidelberg, Germany). Anti-MPO moAb-producing clones were selected by enzyme-linked immunosorbent assay (ELISA) as described previously [105] with minor modifications. Briefly, microtiter plates were coated overnight with murine MPO $(0.5 \mu \mathrm{g} / \mathrm{ml})$, blocked with $1 \%$ bovine serum albumin (BSA), and incubated with undiluted supernatants. Depending on the required isotype, this was followed by incubation with alkaline phosphatase-conjugated goat-anti-mouse antibodies specific for IgG1, IgG2a, or IgG2b (BD Pharmingen, Alphen aan den Rijn, the 
Netherlands). 4-nitrophenyl phosphate (pNPP) was used as substrate, and wells were analyzed spectrophotometrically at $405 \mathrm{~nm}$. Each anti-MPO IgG-producing clone was subcloned 3-5 times. Once the clones were monoclonal, they were grown in Hepes-buffered RPMI Medium 1640 with GlutaMAX ${ }^{\text {TM}}-\mathrm{I}$ enriched with $1 \%$ low-IgG fetal calf serum (Perbio), and 1\% penicillin/streptomycin. Anti-MPO IgG was isolated from the supernatants by protein G column affinity chromatography, after concentration by ultrafiltration, using a $50 \mathrm{kD}$ Amicon filter (Millipore, Amsterdam, the Netherlands). IgG-containing fractions were pooled and dialyzed against PBS, and protein concentrations were determined using the bicinchoninic acid (BCA) protein assay kit (Pierce, Rockford, IL) according to the manufacturer's instructions. Affinity of the moAbs to murine MPO was determined by ELISA as described above. The isotype of each moAb was determined using a mouse isotyping test kit (HBT, Uden, the Netherlands), according to the manufacturer's instructions. A portion of each moAb was biotinylated using standard techniques.

\section{Inhibition ELISA}

To study epitope specificity of the anti-MPO moAbs, we utilized a home-made inhibition ELISA. Briefly, 96-well microtiter plates were coated with murine MPO $(0.5 \mu \mathrm{g} / \mathrm{ml})$ and blocked with BSA. In order to determine the capacity of each moAb (moAb A) to inhibit binding of another moAb (moAb B) to its antigen, the plates were then co-incubated with a fixed dilution of biotinylated moAb B and a two-fold serial dilution of moAb A. To detect the inhibitory effects of competing moAbs, the biotinylated moAb $\mathrm{B}$ was used at the highest dilution giving a maximum OD value by direct ELISA. Non-biotinylated moAb B and an isotype control were used as positive and negative inhibiting controls, respectively. After washing, plates were incubated with peroxidase-labeled streptavidin (Jackson ImmunoResearch, Cambridgeshire, UK). O-phenylene diamine was used as substrate, and plates were read at $490 \mathrm{~nm}$.

\section{Determination of species specificity}

Specificity of the generated antibodies for murine MPO, as well as cross-reactivity with rat MPO was determined by immunohistochemistry on WT, $\mathrm{Mpo}^{-/}$, and rat spleen sections. Sections were acetone-fixed and air-dried, before incubating them with biotinylated anti-MPO moAbs. Endogenous peroxidase activity was blocked with $0.05 \% \mathrm{H}_{2} \mathrm{O}_{2}$ in PBS, and sections were incubated with peroxidase-conjugated streptavidin. Antibody binding was visualized using AEC as substrate, and the slides were counterstained with hematoxylin. Staining intensity was determined semi-quantitatively, using a 0-3+ scale. 
Cross-reactivity of the antibodies to human MPO was determined by indirect immunofluorescence (IIF) on ethanol fixed neutrophils (Inova, San Diego CA, USA) and a direct ELISA on human MPO (Calbiochem, San Diego CA, USA), using fluorescein isothiocyanate (FITC-) labeled rabbit anti-mouse IgG (DakoCytomation, Glostrup, Denmark) and alkaline phosphatase-labeled goat anti-mouse IgG (Jackson ImmunoResearch), as respective conjugates.

\section{INDUCTION OF DISEASE}

Sheep anti-mouse GBM serum was kindly provided by Dr. E. Steenbergen, Dept of Pathology, University Medical Center Nijmegen, the Netherlands. IgG was isolated by 50\% ammonium sulfate precipitation followed by protein $G$ column affinity chromatography. IgG-containing fractions were pooled and dialyzed against PBS. Protein concentrations were determined using the BCA protein assay kit.

In all in vivo experiments, the administered solutions were sterile-filtered before injection $(0.2 \mu \mathrm{m}$ filter; Schleicher \& Schuell, Dassel, Germany). Disease was induced by the intravenous administration of $1 \mathrm{mg}$ sheep anti-mouse GBM IgG, dissolved in PBS, followed after 10 minutes by an intraperitoneal injection of $1 \mathrm{mg}$ anti-MPO moAb or isotype control. As IgG2a isotype control, we used clone OX-27 (mouse anti-rat MHC class I moAb). As IgG2b isotype control, mouse anti-TNP moAb (generously provided by M.R. Daha, Leiden, the Netherlands) was used [169].

In a separate series of experiments, we investigated whether intraperitoneal administration of a single anti-MPO moAb, a combination of several moAbs, or moAbs administered in the context of a systemic proinflammatory response induced by the intraperitoneal administration of $0.5 \mu \mathrm{g} / \mathrm{g}$ lipopolysaccharide (LPS), resulted in NCGN.

\section{LABORATORY AND PATHOLOGICAL EVALUATION OF DISEASE}

At several timepoints during disease development, urine samples were tested for hematuria using dipsticks (Bayer, Mijdrecht, the Netherlands), and scored on a $0-4+$ scale. On the evening prior to the timepoints indicated, mice were placed in metabolic cages for 16 hours to acquire urine for the determination of albuminuria by ELISA (Bethyl, Inc., Montgomery TX). Blood urea nitrogen (BUN) levels were determined in sera collected at the time of sacrifice by an enzymatic degradation assay on a Synchron LX20 PRO (Beckman Coulter Inc., Fullerton CA, USA).

After sacrifice, tissue samples were taken from both kidneys and processed for light microscopy, immunohistochemistry, and immunofluorescence. For light microscopy, renal tissue samples 
were fixed in $4 \%$ formaldehyde and embedded in paraffin. Sections $(1.5 \mu \mathrm{m})$ were cut and hematoxylin/eosin (HE) and periodic acid-Schiff (PAS) staining was performed. For each animal, the amount of crescents was determined in 100 consecutive glomerular cross sections. Only glomeruli that had two or more layers of cells in Bowman's space were considered crescentic. Similarly, for each animal a glomerular PAS-score was determined by counting the mean number of quarters containing PAS-positive material in 100 consecutive glomerular cross sections. Analysis was performed in a blinded manner.

The number of infiltrating leukocytes was determined by immunohistochemistry on $4 \mu \mathrm{m}$ cryostat sections fixed in 100\% acetone at room temperature, using rat anti-mouse CD45 and peroxidase-labeled rabbit anti-rat IgG (DakoCytomation) as primary and secondary antibody, respectively. Endogenous peroxidase activity was blocked with $0.05 \% \mathrm{H}_{2} \mathrm{O}_{2}$ in PBS. Antibody binding was visualized using 3-amino-9-ethylcarbazole (AEC) with $\mathrm{H}_{2} \mathrm{O}_{2}$ as substrate. Sections were counterstained with hematoxylin. Glomerular cell infiltrates were determined by counting the number of positive cells in 30 glomerular cross sections per kidney section.

By immunofluorescence, glomerular cross-sections were stained for the presence of sheep IgG and mouse IgG, using FITC-labeled rabbit anti-sheep IgG (Jackson ImmunoResearch) and Alexa Fluor 488-labeled rabbit anti-mouse IgG (Molecular Probes, Leiden, The Netherlands), respectively. Serum anti-MPO titers were measured in an ELISA as described previously [105], using alkaline phosphatase-labeled goat anti-mouse $\operatorname{Ig} G$ antibodies as conjugate.

\section{STATISTICAL ANALYSIS}

Data are expressed as mean $\pm \mathrm{SD}$ and were analyzed by two-tailed Student's t-test using Graphpad Prism 4.03 for Windows (Graphpad Software, San Diego CA, USA). Because of their non-Gaussian distribution, albuminuria data are compared by two-tailed Mann-Whitney $\mathrm{U}$ test. In both cases, $\mathrm{p}$-values $\leq 0.05$ were considered statistically significant. 


\section{Results}

\section{GENERATION AND CHARACTERIZATION OF ANTI-MPO MOABS}

We generated and characterized eight anti-MPO hybridomas producing moAbs of the IgG1 isotype, two IgG2a moAbs, and one IgG2b moAb. Specificity for mouse MPO was determined by ELISA on purified murine MPO, and confirmed by immunohistochemistry on WT and $M p 0^{-1-}$ spleen sections (Figure 5-1), showing specific staining of neutrophils in WT spleen (Figure 5-1a), and absence of any staining in $M p 0^{-1-}$ tissue (Figure 5-1b). As shown in Table 5-1, several of the moAbs cross-reacted with rat MPO as determined by immunohistochemistry on rat spleen sections, whereas only one moAb (4H9) showed weak cross-reactivity with human MPO by ELISA.

Table 5-1: Anti-MPO moAbs, isotypes, cross-inhibition, and cross-reactivity

\begin{tabular}{|c|c|c|c|c|c|}
\hline Clone & Isotype & Cross-inhibited by & Group & $\begin{array}{l}\text { Cross-reactivity } \\
\text { with rat MPO }\end{array}$ & $\begin{array}{c}\text { Cross-reactivity } \\
\text { with human MPO }\end{array}$ \\
\hline $8 \mathrm{~F} 4$ & IgG1 & $4 \mathrm{H} 9$ & $\mathrm{~A}$ & + & - \\
\hline $2 \mathrm{D} 4$ & $\operatorname{IgG1}$ & $8 \mathrm{~F} 4,4 \mathrm{H} 9,6 \mathrm{G} 4$ & A & +++ & - \\
\hline $4 \mathrm{H} 9$ & $\operatorname{IgG1}$ & 8F4, 6G4, 8F11 & $\mathrm{A}$ & ++ & + \\
\hline $1 \mathrm{~F} 1$ & $\operatorname{IgG1}$ & $\begin{array}{c}\text { 3B1, 1H4, 6G4, } \\
\text { 6D1, 8F11 }\end{array}$ & B & + & - \\
\hline $1 \mathrm{H} 4$ & $\operatorname{IgG1}$ & $\begin{array}{c}\text { 3B1, 1F1, 6G4, } \\
\text { 6D1, 8F11 }\end{array}$ & B & + & - \\
\hline $3 \mathrm{~B} 1$ & $\operatorname{IgG} 1$ & $\begin{array}{c}\text { 1H4, 1F1, 3F7, } \\
6 \mathrm{G} 4,6 \mathrm{D} 1,8 \mathrm{~F} 11\end{array}$ & B & + & - \\
\hline $3 \mathrm{~F} 7$ & $\operatorname{IgG1}$ & 4B3, 6G4 & $\mathrm{C}$ & - & - \\
\hline $4 \mathrm{~B} 3$ & $\operatorname{IgG1}$ & 3F7, 6G4, 6D1 & C & - & - \\
\hline $6 \mathrm{G} 4$ & $\operatorname{IgG} 2 \mathrm{a}$ & $\begin{array}{c}4 \mathrm{H} 9,1 \mathrm{~F} 1,3 \mathrm{~B} 1, \\
1 \mathrm{H} 4,8 \mathrm{~F} 11\end{array}$ & $\mathrm{~A} / \mathrm{B}$ & + & - \\
\hline $8 \mathrm{~F} 11$ & $\operatorname{IgG} 2 \mathrm{a}$ & $\begin{array}{c}4 \mathrm{H} 9,1 \mathrm{~F} 1,3 \mathrm{~B} 1, \\
1 \mathrm{H} 4,6 \mathrm{G} 4\end{array}$ & $\mathrm{~A} / \mathrm{B}$ & +++ & - \\
\hline 6D1 & $\operatorname{IgG} 2 b$ & 1F1, 3B1, 1H4 & B & - & - \\
\hline
\end{tabular}





Figure 5-1: Specificity and cross-reactivity of anti-murine MPO antibodies. Immunohistochemical (a-c) staining of WT (a), $M p^{-\%}$ (b) and rat (c) spleen sections with anti-MPO moAb (clone 8F4 (a and b) and clone 2D4 (c)). Original magnification x200. Immunofluorescence double labeling of clone 8F4 (d, green) and a rat moAb specific for murine neutrophils (clone NIMP-R14; e; red) on WT spleen sections demonstrated co-localization of MPO with neutrophils (f, overlay of pictures $\mathrm{d}$ and $\mathrm{e})$

\section{INHIBITION ELISA}

In order to determine epitope specificity of the generated moAbs, we used an inhibition ELISA, based on the assumption that binding of a moAb to its antigen inhibits binding of another moAb with specificity for a (partly) overlapping epitope. Generally, the inhibition studies revealed a pattern of grouped reciprocal inhibition. In other words, if moAb A inhibits (biotinylated) moAb $\mathrm{B}$, moAb B also inhibits (biotinylated) moAb A, but this reciprocal inhibition only occurs if both antibodies are part of one group, recognizing epitopes that are at least partly overlapping. Roughly, the generated panel of antibodies could be divided into three groups, whereas inhibition occurred within, but not between the groups. A representative example of an inhibition ELISA, showing the binding of biotinylated $8 \mathrm{~F} 4$ to be inhibited by clone $4 \mathrm{H} 9$, but not by the other clones and normal mouse $\mathrm{IgG}$, is presented in Figure 5-2. 


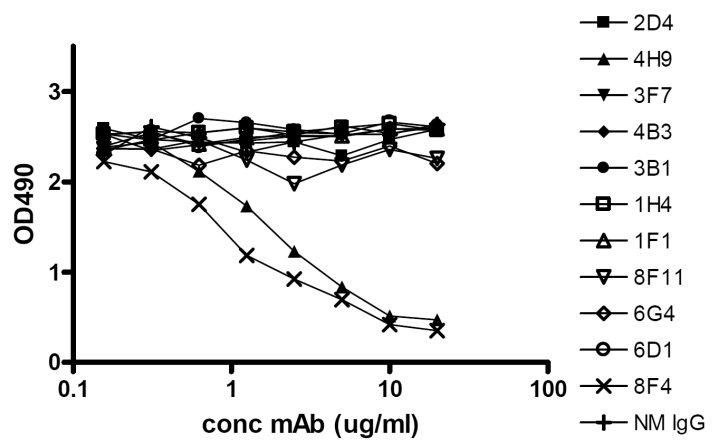

Figure 5-2: Representative example of the inhibition ELISAs summarized in Table 5-1. Reactivity of clone 8F4 is dose-dependently inhibited by clone $4 \mathrm{H} 9$ and unbiotinylated $8 \mathrm{~F} 4$ but not by the other clones generated and normal mouse $\operatorname{Ig} G$

\section{PATHOGENiCITY OF ANTI-MPO MOABS}

\section{Anti-MPO moAbs do not induce NCGN in the context of an LPS-induced systemic proinflammatory stimulus}

We have previously shown that NCGN could be induced by administration of polyclonal antiMPO IgG alone [43], and was markedly aggravated by bacterial lipopolysaccharide [105]. In this study we therefore first investigated the pathogenic effect of the anti-MPO moAbs alone, or in the context of a systemic proinflammatory environment induced by the administration of 0.5 $\mu \mathrm{g} / \mathrm{g}$ LPS. None of the generated moAbs, applied in several dosages, alone or in several combinations of IgG1, IgG2a and/or IgG2b clones, had the capacity to induce NCGN.

\section{Mild anti-GBM glomerulonephritis is severely aggravated by anti-MPO moAbs}

It is already known that a polyclonal anti-MPO immune response can enhance the pathogenicity of anti-GBM antibodies [45, 170,171]. Therefore, we conducted a series of experiments to evaluate the pathogenicity of our anti-MPO moAbs in the context of a local proinflammatory stimulus provided by the intravenous administration of anti-GBM IgG.

In mice injected with polyclonal anti-MPO IgG and LPS [105], we observed that anti-MPO $\operatorname{IgG} 2 \mathrm{a}$ and IgG2b titers decreased faster than the IgG1 titer (Figure 5-3). We hypothesized that this was due to the predominant involvement of $\operatorname{IgG} 2 \mathrm{a}$ and $\operatorname{IgG} 2 \mathrm{~b}$ antibodies in the induction of NCGN by polyclonal anti-MPO IgG. Therefore, we chose to study the effects of 6D1, an antiMPO moAb of the IgG2b isotype, and 6G4, an IgG2a moAb, on mild GN induced by $1 \mathrm{mg}$ sheep anti-GBM IgG.

As shown in Figure 5-4, administration of 6D1 as well as 6G4 caused a marked increase in early albuminuria (37.4 (range 7.4-82.8) and 14.2 (range 5.0-40.5) $\mathrm{mg} / 16 \mathrm{~h}$ in mice receiving 6D1 and 6G4, respectively; $\mathrm{p}<0.05$ compared to the respective control groups) and hematuria. At this time 
point, albuminuria in mice treated with isotype control antibodies was significantly less severe (5.4 (range 0.22-20.7) and 2.7 (range $0.30-5.7$ ) $\mathrm{mg} / 16 \mathrm{~h}$ in mice receiving IgG2b and IgG2a isotype control, respectively). On day 7 , the animals that had received $6 \mathrm{D} 1$ or $6 \mathrm{G} 4$ still had considerable hematuria, whereas this was absent in both control groups (Figure 5-4c). In addition, the animals in both experimental groups still had high levels of albuminuria (30.9 (range 9.0-56.4) and 16.3 (range 8.8-25.1) $\mathrm{mg} / 16 \mathrm{~h}$ in mice receiving 6D1 and 6G4, respectively). The administration of $\operatorname{IgG} 2 \mathrm{~b}$ isotype control resulted in mild albuminuria on day 7 . In contrast, IgG2a isotype control antibody treatment induced albuminuria to a level comparable to the level observed after the administration of 6G4 (Figure 5-4d).

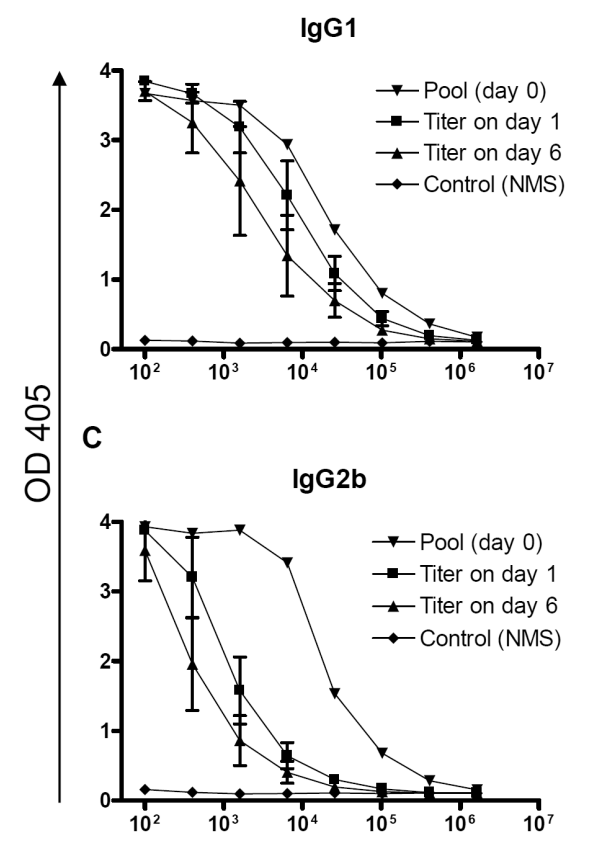

B



D



Dilution

Figure 5-3: Titer of different anti-MPO IgG subclasses in C57Bl/6 mice on several timepoints after the administration of polyclonal anti-MPO IgG and LPS [105]. Whereas IgG1, IgG2a and IgG2b are equally present in polyclonal IgG obtained by immunizing $M p o^{-/-}$mice with murine MPO (d), IgG2a (b) and IgG2b (c) anti-MPO titers decrease faster than the IgG1 (a) anti-MPO titer after injection of polyclonal IgG in combination with LPS. In all sera, including the original pool, IgG3 anti-MPO was not detected (results not shown). Black down-pointing triangle $(\boldsymbol{\nabla})$ : anti-MPO IgG subclass titer in the polyclonal pool, prior to administration; black squares

(匹): serum anti-MPO subclass titer one day after intraperitoneal administration of polyclonal anti-MPO IgG; black up-pointing triangle $(\mathbf{\Delta})$ : serum anti-MPO subclass titer six days after intraperitoneal administration of polyclonal anti-MPO IgG; black diamond ( ) : normal mouse serum (negative control). Results are depicted as mean \pm SD of five animals. In Figure $\mathrm{d}$, the $\operatorname{IgG}$ subclass titers in polyclonal anti-MPO $\operatorname{IgG}$ prior to administration (the black down-pointing triangles in Figure a-c) are plotted to validate the differences in Figure a-c 
After sacrifice on day 7, kidneys were harvested for histopathological and immunohistochemical analysis. As shown in Figure 5-5, both 6D1 and 6G4 severely aggravated mild NCGN induced by a low dose of sheep anti-GBM $\operatorname{IgG}$, whereas their isotype controls only caused mild histopathological alterations. The difference was most remarkable between 6D1 and its IgG2b isotype control: no crescent formation and only minimal glomerular PAS-positivity was discernable in the mice that had received $\operatorname{IgG} 2 \mathrm{~b}$ isotype control whereas in mice that had received 6D1 13.6\% (range 8-17\%) crescentic glomeruli and a glomerular PAS-score of 1.13 (range 0.74-1.48; $\mathrm{p}<0.0001$ compared to isotype control) were observed (Figure 5-6).


Figure 5-4: Urinalysis. On day 1 (a, b) and day 7 (c, d), hematuria (a, c) and albuminuria (b, d) were assessed by dipstick analysis and albumin ELISA, respectively. On day 1 and 7, anti-MPO moAbs 6G4 and 6D1 caused severe hematuria and albuminuria in combination with anti-GBM IgG. On day 7, mice that received IgG2a isotype control antibody also had considerable proteinuria. Black bars: anti-MPO moAbs; white bars: isotype controls

The IgG2a anti-MPO moAb 6G4 also increased disease severity (Figure 5-5). After sacrifice on day 7 , mice that had received anti-GBM IgG and 6G4 had on average 26\% (range 18-36\%; 
$\mathrm{p}<0.0001$ compared to isotype control) crescentic glomeruli, and a glomerular PAS-score of 1.65 (range 1.18-1.87; $\mathrm{p}<0.0001$; Figure 5-6). Surprisingly however, the animals that had received $\operatorname{IgG2a}$ isotype control and anti-GBM IgG also developed some renal morphological abnormalities (Figure 5-5). In this group, on average 1.2\% (range $0-2.0 \% ; \mathrm{p}<0.001$ compared to 6G4) of all glomeruli were crescentic, and the average glomerular PAS-score was 0.44 (range 0.26-0.64; Figure 5-6; $\mathrm{p}<0.001$ compared to $6 \mathrm{G} 4)$.


Figure 5-5: Anti-MPO moAbs 6G4 (IgG2a, fig a, c, d) and 6D1 (IgG2b, fig f, h, i) severely increase glomerular damage in comparison to their isotype controls ( $\mathbf{b}$ and $\mathbf{e}$, and $\mathbf{g}$ and $\mathbf{j}$, respectively) upon administration of a low dose of anti-GBM IgG. Whereas administration of anti-GBM IgG and isotype control induces only mild glomerular PAS-positivity and crescent formation, the addition of $6 \mathrm{G} 4$ or $6 \mathrm{D} 1$ results in severe renal damage, characterized by the deposition of PAS-positive material and the formation of crescents (arrows). PAS staining, original magnification x200 (a, b, f, g); x400 (c-e, h-j) 



Figure 5-6: Anti-MPO moAbs 6G4 and 6D1 severely increase the percentage of crescentic glomeruli (a) and the mean glomerular PAS-positivity (b) in combination with a dose of anti-GBM $\mathrm{IgG}$ that is only mildly nephritogenic in combination with an isotype control

To investigate whether the observed differences in histopathological damage were also reflected in renal function, we determined BUN levels in all mice sacrificed at day 7 , using sera obtained at the time of sacrifice. As can be seen in Figure 5-7, 6G4 and 6D1 significantly increased BUN levels in combination with anti-GBM IgG, whereas their respective isotype controls caused only mild renal function disturbance.

Immunofluorescence staining of renal cross-sections for sheep $\operatorname{IgG}$ revealed a glomerular linear capillary pattern on $\mathrm{t}=3 \mathrm{~h}$ as well as on $\mathrm{t}=7$ days, that was equally intense in all groups sacrificed at the same timepoint (results not shown). At $\mathrm{t}=3 \mathrm{~h}$, a diffuse glomerular mesangial granular staining pattern was observed upon staining for mouse $\operatorname{IgG}$. This was slightly more intense in mice that had received $6 \mathrm{G} 4$ or $6 \mathrm{D} 1$, compared to mice that had received isotype control. Also on day 7, probably due to "trapping" of IgG in the damaged glomeruli, the fluorescence intensity for mouse $\operatorname{IgG}$ tended to be somewhat higher in the experimental groups than in the controls.

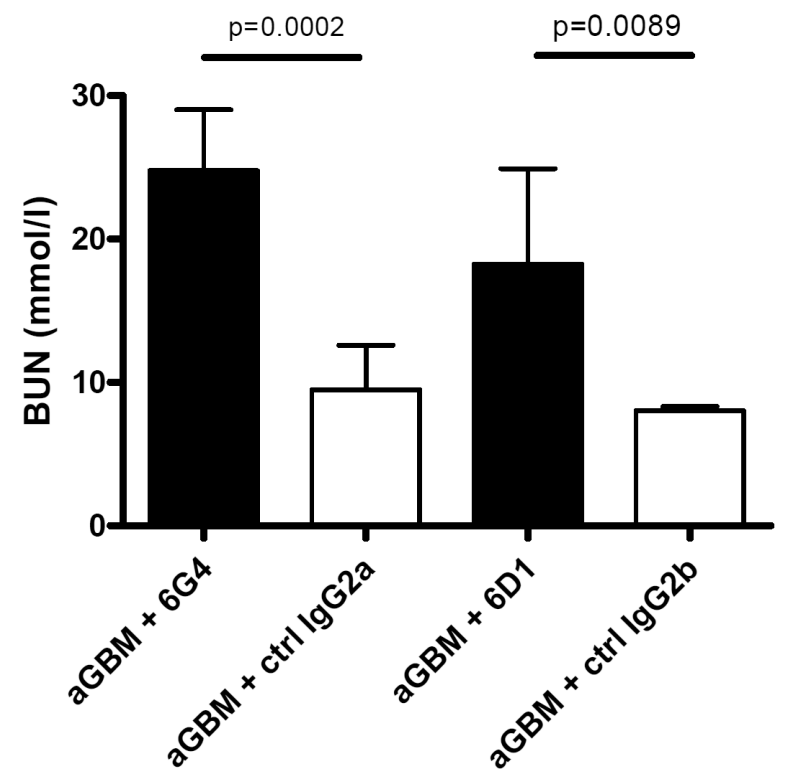

Figure 5-7: Increased blood urea nitrogen (BUN) levels in mice treated with anti-MPO moAbs 6G4 and 6D1 in combination with anti-GBM IgG 
As determined by ELISA and using a total IgG-specific conjugate, no difference was observed in anti-MPO titers between mice receiving 6G4 and mice receiving 6D1, in sera obtained after $3 \mathrm{~h}$, 24h, and 7 days. As expected, no anti-MPO IgG could be detected in mice receiving isotype control moAb (results not shown).

\section{Discussion}

The pathogenicity of polyclonal mouse anti-murine MPO IgG has been well established in the mouse model of anti-MPO IgG-induced NCGN [43, 105, 147]. In this model, as well as in human ANCA-associated vasculitis, it is largely unknown whether there is a subset of ANCA, having a specific isotype and/or recognizing a specific epitope, which is responsible for their pathogenic effect. In this study, we describe the generation and characterization of a panel of mouse moAbs specific for murine MPO, and we show that two of the generated clones are severely pathogenic in the context of a mild anti-GBM glomerulonephritis. Our results provide additional evidence for the pathogenicity of anti-MPO IgG. In addition, our study shows that these moAbs are important tools for further investigations into the role of isotype and/or epitope-related functions of autoantibodies in the pathogenesis of anti-MPO IgG-induced NCGN.

We employed a combination of inhibition ELISAs to obtain an indication of epitope specificity of the generated moAbs. The results from these assays suggest that the moAbs recognize epitopes that are located on three immunoreactive locations on the murine MPO-molecule. This is in line with studies in humans ([172-176], for a review, see [177]), suggesting that MPO-ANCA recognize a restricted number of epitopes on human MPO.

Next, we investigated whether the generated anti-MPO moAbs were cross-reactive with human and rat MPO. Only one of the generated moAbs $(4 \mathrm{H} 9)$ showed weak cross-reactivity with human MPO, indicating that, despite a reported $85 \%$ amino acid similarity between mouse and human MPO [178], the immunoreactive regions of murine MPO are not found on its human counterpart. This result is however in line with the observation that all mouse anti-human MPO moAbs, as well as the vast majority of polyclonal MPO-ANCA derived from human sera, do not cross-react with rat MPO [179]. Conversely, in the study presented here, all except one of the generated anti-MPO moAbs showed cross-reactivity with rat MPO. Apparently, mice and rats are phylogenetically related to such an extent, that the murine immunogenic sites are preserved on the rat MPO molecule. 
In previous studies, we showed that administration of polyclonal anti-MPO IgG causes NCGN [43], which is aggravated by bacterial LPS [105]. However, the anti-MPO moAbs generated in this study did not cause NCGN when administered alone, in combinations, and/or in combination with LPS. This is in contrast with mouse models of autoimmune arthritis [180-182] and pemphigus vulgaris [183, 184], in which both polyclonal and monoclonal autoantibodies induce disease in the recipient animals.

It is unclear why the anti-MPO moAbs in our study, alone or in various combinations, with or without LPS, do not exert the same in vivo effect as polyclonal anti-MPO IgG. One possibility is that we might not yet have selected a clone recognizing a pathogenic epitope. Alternatively, pathogenicity might be restricted to a monoclonal antibody with a highly specific combination of proper isotype and epitope specificity. Since we selected only two IgG2a and one IgG2b moAb, a pathogenic anti-MPO moAb might be having either of those isotypes, but another epitope specificity.

The administration of an IgG2a or IgG2b anti-MPO moAb led to severe aggravation of mild anti-GBM disease, as determined by severe urinary abnormalities, histopathological damage, and loss of renal function after 7 days. These results are in line with a study in rats revealing an aggravating effect of rabbit anti-rat MPO IgG on anti-GBM glomerulonephritis [170], and with two studies demonstrating that administration of anti-GBM IgG results in more severe disease if the recipient animals are first immunized with human MPO $[45,171]$. In these studies however, the observed effect was exerted by polyclonal antibodies, whereas in our study, we describe an effect of monoclonal anti-MPO antibodies.

Anti-MPO IgG2a and IgG2b titers are rapidly declining upon the administration of polyclonal anti-MPO IgG in combination of LPS, whereas the anti-MPO IgG1 titer remains high. This suggests that in polyclonal anti-MPO IgG, IgG2a and IgG2b are responsible for disease development. Apart from a more rapid clearance of anti-MPO IgG2a and IgG2b from the sera of mice injected with polyclonal anti-MPO-IgG, there are several other reasons to assume a predominant role of IgG2a and IgG2b autoantibodies in MPO-ANCA-mediated disease. Firstly, preliminary data show that in the mouse model of anti-MPO IgG-induced NCGN, deficiency of both $F_{c} \gamma$ receptors $F_{c} \gamma R I$ and $F_{c} \gamma R I I I$ is required to prevent polyclonal anti-MPO IgG-induced disease development [185]. A similar pattern is observed in a model of murine experimental autoimmune hemolytic anemia, in which the pathogenicity of various isotype switch variants of a murine moAb causing hemolytic anemia is studied in several $F c \gamma R$-deficient mouse strains [186]. In this study, pathogenicity of the $\operatorname{IgG} 2 \mathrm{a}$ and $\operatorname{IgG} 2 \mathrm{~b}$ isotype-switch variants is only fully 
prevented in common gamma-chain $(\mathrm{FcR} \gamma)$ deficient mice, that do not express the $\mathrm{Fc} \gamma \mathrm{RI}$ as well as the FcyRIII receptor. This is in accordance with the observations in murine polyclonal antiMPO IgG-induced NCGN [185], and provides indirect support for the pathogenicity of antiMPO IgG2a and IgG2b. However, the exact role of the IgG isotype remains to be determined, using IgG1 clones with exactly the same epitope specificity as 6B1 and 6G4.

Another pathogenicity-determining characteristic determined by the isotype of a moAb, lies in its capacity to activate complement. In the mouse model of anti-MPO IgG-induced NCGN, it has been shown that activation of the alternative complement pathway is pivotal in disease development $[148,187]$. It would therefore be interesting to study the complement-activating potential of moAbs that share a similar idiotype, but have different isotypes. In this respect, it has been shown in the mouse model of hemolytic anemia described above, that IgG2a and IgG2b, but not IgG1 isotype-switch variants bind complement factor C3 in vivo [188]. Whether antiMPO IgG2a and/or IgG2b are also better activators of the complement pathway than anti-MPO $\operatorname{Ig} \mathrm{G} 1$, is as yet unknown.

An unexpected observation in our study was the mild increase in disease severity induced by the administration of control IgG2a after the injection of anti-GBM antibodies, when compared to the control group receiving $\operatorname{IgG} 2 \mathrm{~b}$. Additional studies are necessary to explain the observed effect of this irrelevant IgG2a moAb on anti-GBM glomerulonephritis.

In conclusion, the study presented here describes the generation and characterization of a panel of moAbs specific for mouse MPO. The generated clones are not pathogenic in combination with LPS, but an IgG2a and an IgG2b anti-MPO moAb severely aggravate mild anti-GBM glomerulonephritis. Thus, a new murine model is provided that can be used to specifically address the importance of several antibody effector functions related to isotype, idiotype, and possibly to other antibody properties, in the development of anti-MPO IgG-induced NCGN. Studying this model may help us to obtain a better understanding of the pathophysiology of human MPO-ANCA associated vasculitis and glomerulonephritis. 



\section{Chapter 6-MPO is critically involved in the induction of organ damage after renal ischemia reperfusion}

Submitted as part of: Robert A. Matthijsen, Dennis Huugen, Nicole T. Hoebers, Bart de Vries, Carine J. Peutz-Kootstra, Yasuaki Aratani, Jan Willem Cohen Tervaert, Wim A. Buurman and Peter Heeringa. Myeloperoxidase is critically involved in the induction of organ damage following renal ischemia reperfusion. 


\section{Abstract}

Injury induced by renal ischemia and reperfusion (IR) is one of the factors that are still complicating not only renal transplantation surgery, but also a variety of other clinical conditions. Myeloperoxidase (MPO) is a neutrophil enzyme that is not only involved in host defence, but also in several immune-mediated inflammatory syndromes. In the present study, we utilized a murine model of renal IR injury to evaluate the role of MPO in this condition.

Renal IR injury was applied in wildtype (WT) and myeloperoxidase-deficient $\left(\mathrm{Mpo}^{-/}\right)$mice by unilateral clamping for 40 minutes, followed by removal of the contralateral kidney and 24 hours of reperfusion. Blood urea nitrogen (BUN) levels were determined to evaluate the extent or renal damage, and immunohistochemistry was utilized to investigate neutrophil influx and renal deposition of MPO.

24 hours after reperfusion, the mean BUN level in $M p o^{-/}$mice was $31.4 \pm 3.9$, compared to $42.8 \pm 5.2$ in WT mice $(\mathrm{p}=0.003)$. In addition, renal neutrophil influx was significantly lower in $M p 0^{-/-}$compared to WT mice $(28.2 \pm 7.6 \mathrm{vs}$. $40.4 \pm 5.2$ cells per high-power field, $\mathrm{p}<0.02)$.

From the data presented here, we conclude that MPO might play a hitherto unrecognized role in renal IR injury in humans. Finally, we discuss several potential mechanisms through which this versatile enzyme might be involved in renal IR. 


\section{Introduction}

In clinical medicine, complications arising from organ ischemia and reperfusion (IR) are common phenomena, complicating the treatment of severely injured or ill patients and influencing the outcome of various clinical conditions. Our understanding of the pathophysiology of IR induced organ damage is limited, and this impairs the development of new and effective therapies.

In renal IR injury, cellular injury is one of the potential targets that has received much interest over the last decades. It is induced by ischemia and aggravated upon reperfusion, and forms a potent trigger for the activation of an extensive inflammatory response, illustrated by the production of various cytokines such as TNF- $\alpha$ and the interleukins 10, 12 and 18 [189, 190], the infiltration and activation of polymorphonuclear neutrophils (PMNs) in the affected area, as well as the expression or deposition of various components of the innate immune response, such as complement factors [191]. Under healthy conditions, the cells and proteins orchestrate a wellmounted attack on invading micro-organisms, but when faced with extensive IR injury, they seem to lack sufficient means of control.

MPO is a $140 \mathrm{kD}$ heme protein that is predominantly stored in the lysosomes of monocytes and in the azurophilic granules of resting PMNs [20]. It is one of the enzymes that are released upon neutrophil activation, and its capacity to catalyze the formation of hypochlorite ( $\mathrm{HOCl}$ ) out of hydrogen peroxide $\left(\mathrm{H}_{2} \mathrm{O}_{2}\right)$ and chloride ions makes it a powerful tool in the bactericidal armament of these cells [20]. However, there are also clinical studies indicating a potentially harmful effect in immune-mediated inflammatory syndromes, such as multiple sclerosis [192, 193], acute coronary syndrome [194], and renal disease (reviewed in [19]). In addition, a considerable line of research indicates that MPO and MPO-derived oxidants are involved in the pathogenesis of atherosclerosis, possibly through catalyzing the oxidation of low-density [195199] and high-density [200] lipoproteins.

It can be concluded from these studies that MPO can have both beneficial and detrimental effects in a variety of immune-mediated diseases. However, although the immune system is generally thought to be also involved in renal IR, the role of MPO in this is as yet unknown. More information regarding this issue might teach us more about the pathophysiological mechanisms that are responsible for renal IR, and provide potential targets for therapeutic intervention. Thus, in this chapter, we investigated the role of MPO in a mouse model of renal unilateral ischemia reperfusion injury, by comparing disease severity in wildtype (WT) and MPOdeficient $\left(M p o^{-1}\right)$ mice with respect to various disease parameters. Our results show that that in 
this model, MPO-mediated cytotoxicity is in part responsible for the development of renal damage resulting from ischemia and reperfusion. The potential mechanisms through which MPO might exert this effect on murine and human renal IR are discussed.

\section{Methods}

\section{MICE}

$\mathrm{Mpo}^{-/-}$mice, backcrossed to a C57BL/6 background for six times, were genotyped using PCRamplified DNA from tail clippings [61]. Wildtype male C57Bl/6 (11 weeks of age) mice were obtained from Charles River Breeding Laboratories (Heidelberg, Germany). Mice were kept according to University of Maastricht animal facility regulations, and all experiments were approved by the local Animal Care and Experimentation Committee.

\section{EXPERIMENTAL PROCEDURE}

Experiments were carried out as previously described, with minor modifications [201]. At the start of the experiments, mice ( $n=5-6$ per group) were anesthetized with sodium pentobarbital $\left(100 \mathrm{mg} / \mathrm{kg}\right.$ i.p.). Body temperature was maintained at $39^{\circ} \mathrm{C}$ by a heating pad until animals recovered from anesthesia. Under aseptic conditions a $1.0-\mathrm{cm}$ long midline abdominal incision was made and ischemia was induced by applying a non-traumatic vascular clamp to the left renal pedicle for $40 \mathrm{~min}$. Subsequently, the wound was covered with cotton soaked in sterile PBS. Immediately after removal of the clamp, the left kidney was inspected for restoration of blood flow and the contralateral kidney was removed. This provided us with a model of renal transplantation that allowed us to study the effect of renal IR unaffected by changes caused by the acute disruption of renal function prior to reperfusion, as would be the case if a contralateral nephrectomy were performed before the application of ischemia. The wound was closed in two layers, and animals were sacrificed 2 or 24 hours after reperfusion. In mice that were to be sacrificed after 24 hours, $0.25 \%$ bupivacaine was applied topically for postoperative pain management. At the time of sacrifice, plasma was collected and the left kidney was harvested for immunohistochemical and immunofluorescence analysis.

\section{LABORATORY AND PATHOLOGICAL EVALUATION OF DISEASE}

In mice sacrificed after 24 hours, blood urea nitrogen (BUN) levels were determined by an enzymatic degradation assay on a Synchron LX20 PRO (Beckman Coulter Inc., Fullerton CA), in sera collected at the time of sacrifice. Renal neutrophil influx was determined using a rat anti- 
mouse neutrophil antibody (clone NIMP-R14 [78]) on $4 \mu \mathrm{m}$ snap-frozen tissue sections. After incubation with the primary antibody, endogenous peroxidase activity was blocked with $0.05 \%$ $\mathrm{H}_{2} \mathrm{O}_{2}$ in PBS, and rabbit anti-rat IgG-PO and goat anti-rabbit IgG-PO (both DakoCytomation) were then used as secondary and tertiary antibodies, respectively. Antibody binding was visualized using 3-amino-9-ethylcarbazole (AEC) with $\mathrm{H}_{2} \mathrm{O}_{2}$ as substrate. Sections were counterstained with hematoxylin, and juxtamedullary neutrophil influx was quantified by counting the average number of NIMP-R14-positive cells in 10 adjacent high-power fields, located around the medulla.

To determine colocalization of renal MPO deposits with the localization of PMNs, renal crosssections were double-stained with a biotinylated mouse moAb specific for murine MPO (clone 8F4 [105]) and NIMP-R14, using Alexa Fluor 488-labeled streptavidin and Alexa 568-labeled goat-anti-rat IgG (both obtained from Invitrogen, Breda, The Netherlands) as respective conjugates. No significant staining was detected in slides incubated with control rat IgG (NIMPR14) or mouse IgG (8F4).

Plasma MPO levels were determined by in-house catching ELISA as described previously [105]. Briefly, microtiter plates were coated with Fc $\gamma$ fragment-specific goat anti-mouse IgG (Jackson ImmunoResearch), incubated for 48 hours at $4^{\circ} \mathrm{C}$, and blocked with $1 \% \mathrm{BSA}$ in PBS. Plates were then incubated with an anti-murine MPO-specific moAb clone 8F4), followed by incubation with appropriately diluted plasma samples. Next, the plates were incubated with polyclonal rabbit antihuman MPO (DakoCytomation, Glostrup, Denmark) and alkaline phosphatase-labeled polyclonal goat anti-rabbit IgG as primary and secondary detection antibody, respectively. 4Nitrophenyl phosphate (pNPP) was used as substrate, and results were analyzed spectrophotometrically at $405 \mathrm{~nm}$. Concentrations were calculated from a standard curve of purified murine MPO (range, 2.5-100 ng/ml).

\section{STATISTICAL ANALYSIS}

Data are expressed as means $\pm \mathrm{SD}$ and were analyzed by unpaired two-tailed Student's t-test, using Graphpad Prism 4.01 for Windows (Graphpad Software, San Diego CA). P-values $<0.05$ were considered statistically significant.

\section{Results}

To investigate the effect of MPO-deficiency on renal function deterioration induced by renal IR injury, we determined BUN levels in plasma obtained at the time of sacrifice, 24 hours after 
reperfusion and contralateral nephrectomy. As shown in Figure 6-1, $M p o^{-1-}$ mice displayed a markedly less pronounced increase in BUN levels, when compared to WT controls (mean, $31.4 \pm$ 3.9 vs. $42.8 \pm 5.2 \mathrm{mmol} / 1$ in $\mathrm{Mpo}^{-/}$and WT mice, respectively, $\left.\mathrm{p}=0.003\right)$. This was accompanied by a lower degree of renal influx of NIMP-R14-positive PMNs, as determined by immunohistochemistry (Figure 6-2, Figure 6-3, d and h). Whereas WT mice had on average 40.4 \pm 5.2 NIMP-R14-positive cells per high-power field, the mean number of PMNs in $\mathrm{Mpo}^{-/-}$mice was $28.2 \pm 7.6$ per high-power field $(\mathrm{p}<0.02$, Figure 6-2).

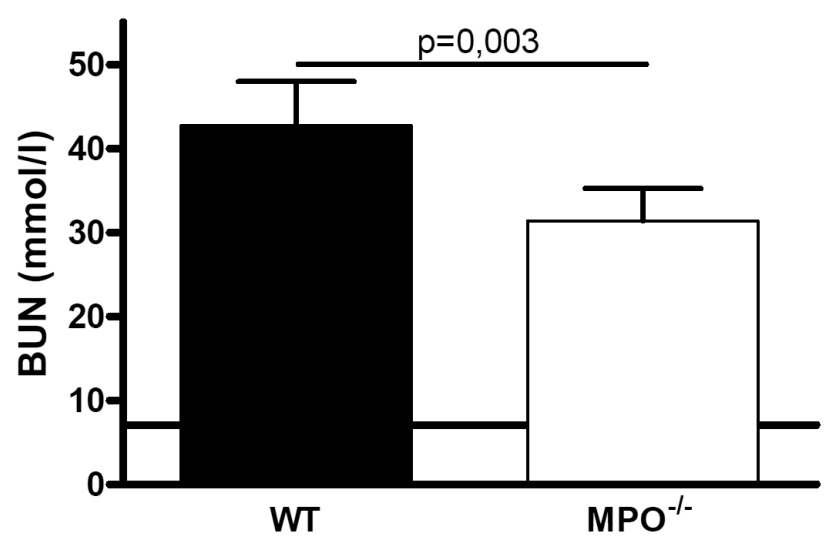

Figure 6-1: MPO-deficient mice are partly
protected from loss of renal function
induced by renal ischemia perfusion, as
reflected by a smaller increase in blood
urea nitrogen (BUN) level after 24 hours.
Untreated WT and Mpo mice had
similar BUN levels; the solid line
indicates the mean BUN level in
untreated mice $(\mathrm{n}=5)$.

To investigate whether MPO-positivity was restricted to PMNs, or whether also extracellular MPO could be found, double-staining with NIMP-R14 and 8F4 was performed. As displayed in Figure 6-3, sequestrated PMNs in WT mice were predominantly positive for the NIMP-R14 antigen as well as MPO. A small amount of MPO-positivity did however not merge with positivity for NIMP-R14, suggesting that extracellular MPO was present in the lesions as well. As expected, no MPO positivity was found in the kidneys of $M p o^{-1-}$ mice (Figure 6-3).

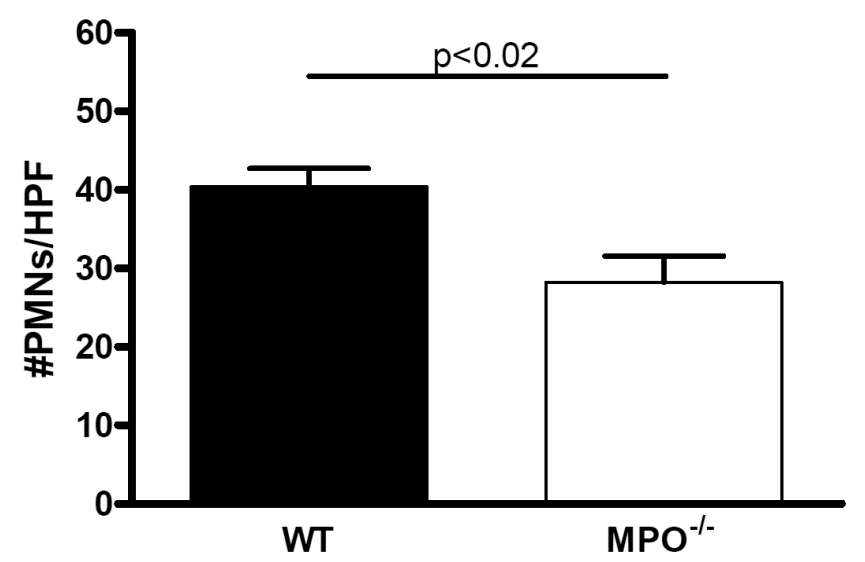

Figure 6-2: Juxtamedullary sequestration of NIMP-R14positive PMNs is attenuated in mice deficient for MPO. 
Apart from the generation of reactive oxygen species, activation of PMNs also induces the release of their granule constituents such as MPO. Indeed, in plasma obtained from WT mice 24 hours after reperfusion, we found significantly elevated levels of circulating MPO compared to untreated WT mice (mean, $24.2 \pm 2.3$ and $11.4 \pm 2.8 \mathrm{ng} / \mathrm{ml}$ in IR and control mice, respectively; $\mathrm{p}<0.0001 ;$ Figure 6-4).

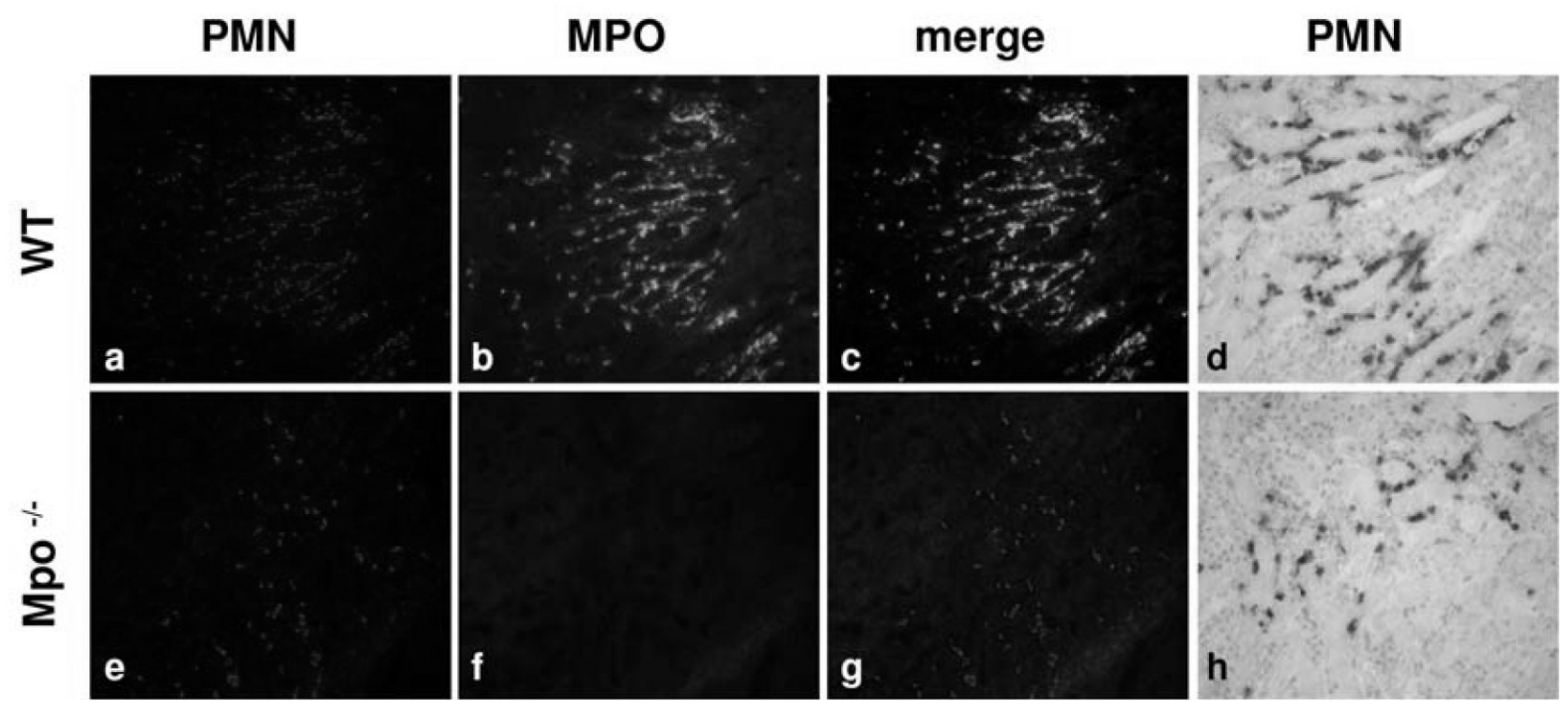

Figure 6-3: Immunofluorescence (a-c and e-g) and immunohistochemistry ( $\mathrm{d}$ and h) pictures of renal tissue stained for PMNs (NIMP-R14, red, a and e) and MPO (8F4, green, b and f). In WT mice, renal deposition of MPO largely colocalizes with NIMP-R14 (c), whereas no MPO is detected after renal IR in Mpo-/ mice (g).

\section{Discussion}

Many of the mechanisms responsible for renal failure after a period of ischemia followed by reperfusion are as yet unknown. In the present study we show that MPO-deficient mice are partly protected from IR-induced renal failure. Therefore, we postulate that this enzyme plays a hitherto unrecognized role in the pathogenesis of this syndrome.

MPO is a neutrophil enzyme that is predominantly stored in the azurophilic granules of PMNs. Its capacity to catalyze the formation of $\mathrm{HOCl}$ out of $\mathrm{H}_{2} \mathrm{O}_{2}$ and chloride-ions is well-documented (for a review, see [20]). It has also been demonstrated in vitro that MPO mediates CD11b/CD18 (MAC-1) dependent adhesion of PMNs [21], and that this specific binding of MPO to CD11b/CD18 results in PMN activation [22]. Finally, the role of MPO as an antigen in vasculitis and glomerulonephritis mediated by MPO-specific anti-neutrophil cytoplasmic autoantibodies (ANCA) is nowadays firmly established [18]. 
In our present study, we showed that the protective effect of MPO-deficiency, as measured by a decrease in BUN levels, is accompanied by a decrease in IR-induced renal influx of PMNs. Bearing in mind the in vitro role of MPO in CD11b/CD18-mediated PMN adhesion and activation $[21,22]$, it is tempting to postulate that the protective effect of MPO-deficiency is mediated by its effect on PMN adhesion and/or activation. In line with this hypothesis, Rabb and colleagues showed a protective effect of CD11b/CD18 blockade in a rat model of acute renal failure [202]. However, the role of MPO in PMN adhesion in vivo is as yet unclear, and also the role of PMNs in renal IR injury is not undisputed ([201, 203-205], and reviewed in [206]). Thus, PMN influx and CD11b/CD18 seem to play an intriguing but complicated role in renal IR, and it is as yet unclear whether MPO has a modifying effect on their action.

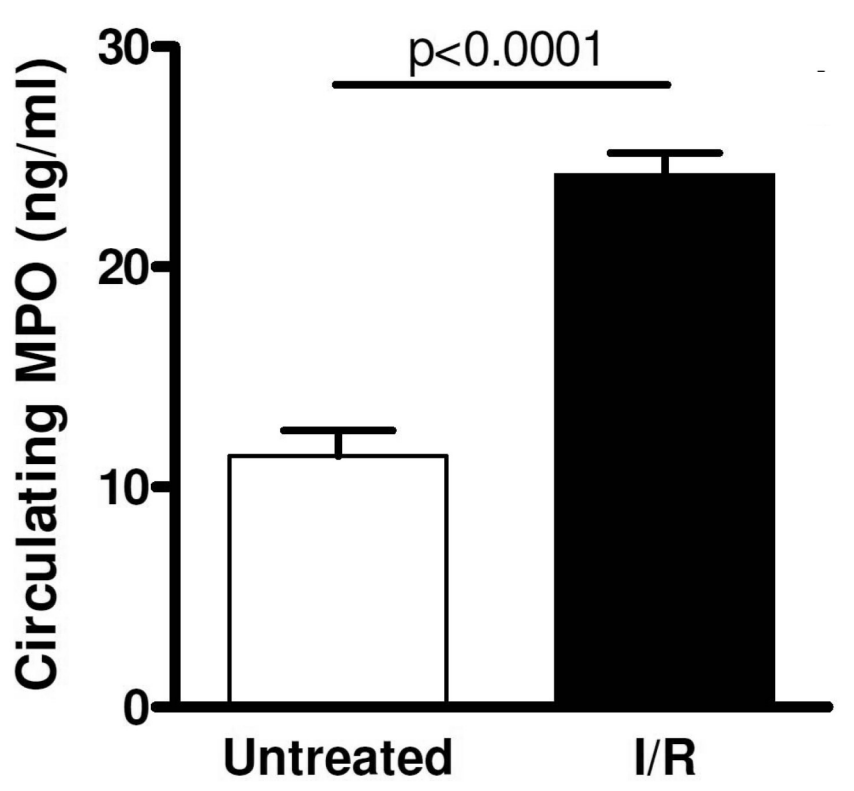

Figure 6-4: Renal IR leads to
an increase in circulating MPO
levels 24 hours after
reperfusion.

Apart from its effect on PMN adhesion and activation, MPO could also be involved in other pathways that play a role in the pathogenesis of renal IR. Firstly, there is convincing in vitro evidence that MPO also plays a role in apoptosis [23-25]. Over the years, a massive body of literature has emerged describing activation of apoptotic pathways in renal IR (reviewed in [207] and [208]). Moreover, inhibition of apoptosis through administration of the anti-apoptotic agent IGF-1 or ZVAD-fmk (a caspase inactivator) [209], repletion of guanosine [210], or administration of a p53 inhibitor [211] has been shown to preserve renal function after renal IR. We are currently testing the hypothesis that in our study, a decrease in MPO-mediated apoptosis is one of the mechanisms through which $\mathrm{Mpo}^{-1-}$ mice are protected from injury caused by renal IR. 
Secondly, a direct activating effect of MPO on complement factor C5 [26, 27] provides a potential mechanism through which MPO plays a role in renal IR injury. It has been shown that inhibition of C5b-9 (MAC) formation [212], as well as treatment with a C5a receptor antagonist [201] attenuates disease in mouse models of renal IR. However, data on a direct effect of MPO on complement system activation are restricted to in vitro studies $[26,27]$.

MPO has been studied as a possible pathogenic factor in a number of other mouse models. In a study on myocardial ischemia, MPO-deficient mice are partly protected against leukocyte influx and myocardial rupture, and have a better post-ischemic left-ventricular function [213]. It is suggested that the effect of MPO in this model is mediated by its oxidizing effect on plasminogen activator inhibitor-1 (PAI-1), resulting in a decreased plasmin activity in $M p o^{-/}$mice. Alternatively, MPO might be involved in myocardial IR injury via the generation of cytotoxic aldehydes [214]. Nevertheless, it remains to be determined whether these mechanisms also play a role in renal IR injury.

Whereas myocardial IR injury is attenuated in MPO-deficient mice, MPO-deficiency is actually detrimental in murine atherosclerosis [215] and murine experimental autoimmune encephalitis [216]. Moreover, $M \mathrm{Po}^{-1-}$ mice also display an increased infarct size in a murine model of cerebral IR injury [217]. It is suggested in these studies that MPO-mediated, oxidative inactivation of proinflammatory molecules [215], or increased proliferation of antigen-specific T-cells in $M p 0^{-1-}$ animals [216] are responsible for the observed effects. Alternatively, as discussed by Nauseef [218], MPO might by protective in these models via the terminating effect of the $\mathrm{MPO}-\mathrm{H}_{2} \mathrm{O}_{2}-$ halide system on the NADPH-oxidase pathway [219]. However, the role of these mechanisms in the pathogenesis of renal IR injury remains obscure.

In conclusion, the study presented here provides evidence that MPO plays a detrimental role in murine renal IR injury. We are currently trying to unravel if this effect is caused by the role of MPO in PMN adhesion and activation, apoptosis, complement pathway activation, plasmin activity, the generation of toxic aldehydes, and/or other, as yet unknown, factors. 

Chapter 7 - Summary and discussion 
Wegener's Granulomatosis, Churg-Strauss Syndrome, microscopic polyangiitis, and idiopathic pauci-immune necrotizing crescentic glomerulonephritis (NCGN), have many characteristics in common. First, their diagnosis is based histopathologically on a sterile inflammation predominantly affecting the arterioles, venules and capillaries of the circulatory system. Second, their presence is often accompanied by elevated levels of anti-neutrophil cytoplasmic autoantibodies (ANCA), predominantly specific for proteinase 3 (PR3) or myeloperoxidase (MPO). Therefore, these diseases are collectively called ANCA-associated small-vessel vasculitides [2].

The close association between the presence of ANCA and vasculitis has led many researchers to investigate their role in the pathogenesis of this disease. In the presented thesis, we try to elucidate the effects of MPO-ANCA -the autoantibodies- as well as MPO -the autoantigen- in renal inflammation. To this end, we utilized the mouse model of anti-MPO IgG-induced glomerulonephritis, as discussed in the next paragraph. In addition, we used the mouse model of renal ischemia reperfusion injury to obtain a more profound insight into the role of MPO in the pathophysiology of (renal) inflammation in a broader sense.

\section{Translation of in vitro observations to in vivo evidence for MPO-ANCA pathogenicity}

As discussed in Chapter 1, a considerable body of in vitro studies generally points towards a mechanism in which the development of full-blown ANCA-associated vasculitis relies on three key players (Figure 7-1). The first player is the polymorphonuclear neutrophil (PMN), being the most prominent source of MPO, PR3, and other potential ANCA antigens. The second is a proinflammatory environment, typically created in vitro by adding a priming concentration of TNF $\alpha$ to the system. This "pre-activates" the PMNs, leading to the upregulation of adhesion molecules and, importantly, to an increased expression of the ANCA antigens on the outer membrane of circulating PMNs and monocytes. ANCA then act as the third player when they bind to primed PMNs, resulting in their activation and the release of lytic granule constituents and reactive oxygen radicals (reviewed in [18]). The adherence of activated PMNs to susceptible endothelium then initiates an inflammatory cascade that ultimately results in symptomatology as clinically observed. 


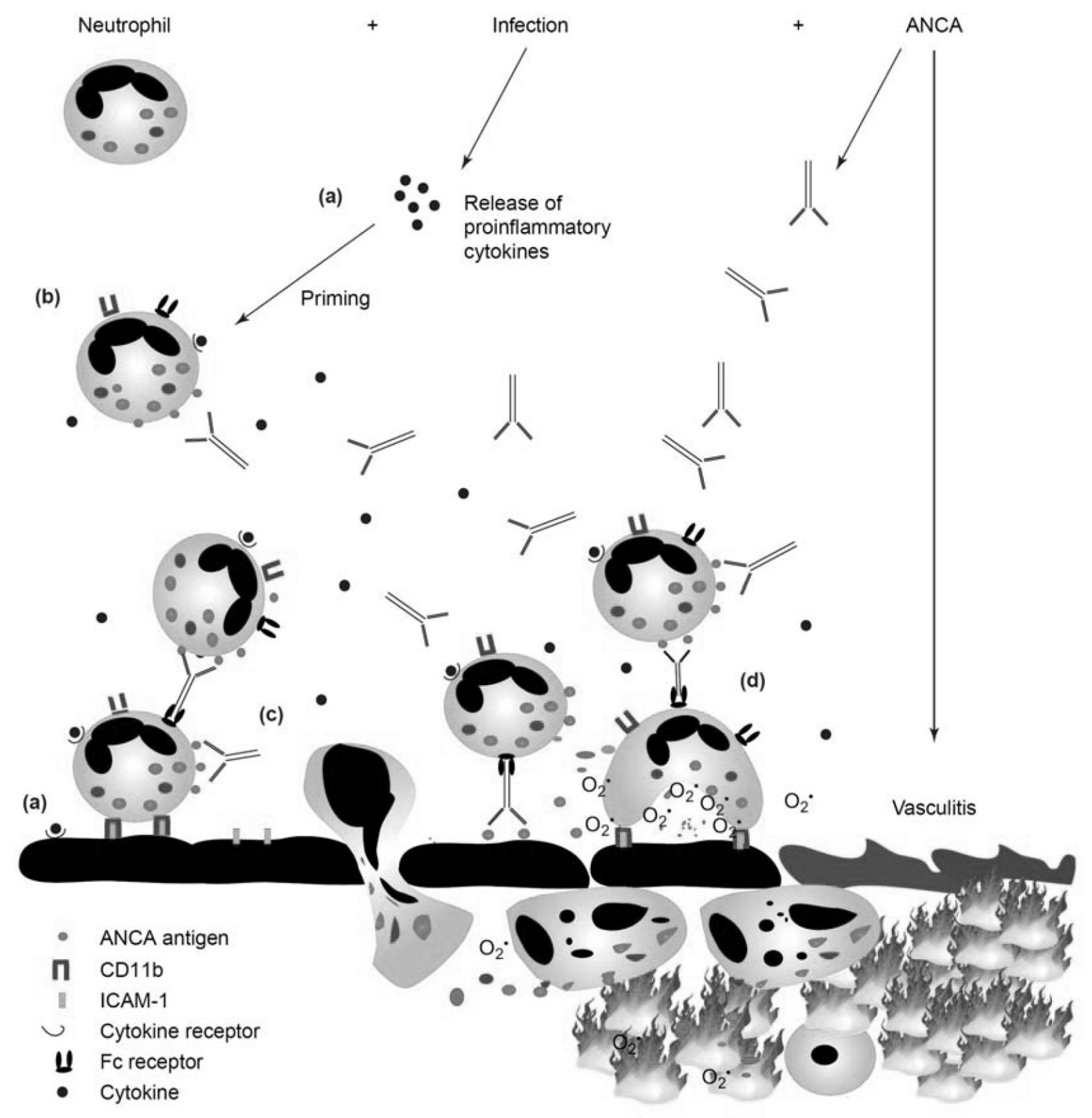

Figure 7-1: Representation of ANCA-mediated neutrophil responses that are putatively involved in the pathogenesis of ANCA-associated small vessel vasculitis. (a) Proinflammatory cytokines and chemokines (e.g. tumor necrosis factor $\alpha$ ), released as a result of local or systemic infection, cause upregulation of endothelial adhesion molecules (e.g. selectins, ICAM-1, and VCAM) and prime the neutrophil. (b) Neutrophil priming causes upregulation of neutrophil adhesion molecules (CD11b) and translocation of the ANCA antigens from their lysosomal compartments to the cell surface. (c) Engagement of the $\mathrm{F}\left(\mathrm{ab}^{\prime}\right) 2$ portion of ANCA with ANCA antigens on the cell surface, and interaction of the Fc part of the antibody with Fc receptors, activates the neutrophil, causing increased neutrophil-vessel wall adherence and transmigration. (d) ANCA-mediated neutrophil activation also triggers reactive oxygen radical production and possibly causes neutrophil degranulation, with consequent release of proteolytic enzymes, leading to vasculitis. 
To test the hypothesis that ANCA-induced activation of primed neutrophils underlies pauciimmune NCGN, the first requisite is an animal model that convincingly proves the pivotal role of ANCA in the pathogenesis of ANCA-associated vasculitis. In Chapter 1, several rodent models addressing this issue are discussed. Although these models generally support the suggested hypothesis, they do not prove unequivocally that ANCA cause vasculitis and glomerulonephritis. In 2002, Xiao and colleagues showed that the adoptive transfer of IgG from murine MPO (muMPO) immunized MPO-knockout $\left(M p o^{-1}\right)$ mice to wildtype (WT) mice resulted in a mild vasculitis and glomerulonephritis, with remarkable similarity to human MPOANCA associated disease [43]. In addition, it has recently been shown that the immunization of WKY rats with human MPO induces the generation of anti-human MPO antibodies that crossreact with rat MPO and cause a mild form of NCGN [49]. Together, these studies do not only prove that MPO-ANCA cause vasculitis and glomerulonephritis, but also provide an exquisite opportunity to further explore the mechanisms involved.

As described above, the transfer of anti-MPO IgG to WT mice only induces mild NCGN. In Chapter 2, we show that bacterial lipopolysaccharide (LPS), being a model proinflammatory stimulus, increases the severity of NCGN induced by anti-MPO IgG in a dose-dependent fashion. This provided us with a disease model that not only more closely resembled human MPO-ANCA associated NCGN, but that was also excellently suitable to investigate the effect of therapeutic intervention on various parameters of disease severity.

We investigated several factors that might be mediating the observed effect of LPS. First, we showed that LPS increases the anti-MPO IgG-induced early (day 1) glomerular influx of PMNs, as well as the late (day 6) influx of several types of leukocytes, in a synergistic fashion. Second, systemic administration of LPS resulted in an immediate, transient increase in serum TNF- $\alpha$ levels, as well as circulating MPO levels. Third, we were able to confirm ANCA-induced activation of PMNs in the mouse model by showing that incubation of murine TNF $\alpha$-pretreated peritoneal exudate cells (predominantly PMNs) with anti-MPO IgG results in a modest but significant increase in superoxide production. Together, these results strongly suggest that in mice, the LPS-induced release of TNF $\alpha$ and/or MPO augments anti-MPO IgG-induced activation of PMNs, thus leading to more severe disease. The activated PMNs then adhere to the glomerular endothelium, ultimately resulting in glomerulonephritis. Importantly, however, we also showed that pretreatment of the animals with a TNF $\alpha$-inhibiting monoclonal antibody (moAb) attenuates, but not fully prevents, disease development. This indicates that other - 
TNF $\alpha$-independent- mechanisms might be more crucially involved in the pathogenesis of antiMPO IgG-induced disease.

In order to prove that anti-MPO IgG-induced activation of primed PMNs causes glomerulonephritis, it is necessary to not only provide evidence for the role of anti-MPO IgG and priming, but also for a crucial role of PMNs in the pathogenesis of anti-MPO IgG-induced NCGN. We addressed this issue by investigating the effect of neutrophil-depletion in the mouse model of anti-MPO IgG-induced disease. In line with the hypothesis, pretreatment of animal with the neutrophil-depleting moAb NIMP-R14 [78] completely inhibited disease development upon administration of anti-MPO IgG [147].

In Chapter 3, we review in vitro and animal studies, as well as clinical data on TNF $\alpha$ and TNF $\alpha$ bioactivity inhibition in ANCA-associated vasculitis, and discuss the potential benefit that patients with ANCA-associated vasculitis might have from TNF $\alpha$ bioactivity-inhibiting therapy. As already briefly discussed in chapters 1 and 2, in vitro studies as well as various animal models strongly suggest an important role for TNF $\alpha$-induced neutrophil priming in the pathogenesis of ANCA-associated vasculitis. In human ANCA-associated disease, however, the therapeutic potential of TNF $\alpha$ inhibition is still controversial. The results from a large, but uncontrolled, study on the effect of infliximab in the treatment of ANCA-associated disease were promising, although major side-effects occurred [87]. However, the first controlled trial on the use of etanercept in this disease was disappointing, since no benefit of adding etanercept to standard therapy could be seen [126]. Moreover, also in this trial major side-effects occurred in the group receiving etanercept.

In summary, it can be concluded from the mouse studies that, in a fully homologous system, antiMPO IgG, an inflammatory environment and PMNs indeed act together in causing full-blown NCGN. However, TNF $\alpha$ plays a less crucial role in the involved mechanisms than could be expected from in vitro observations. Clinical trials, demonstrating that ANCA-associated disease can perpetuate and relapse during TNF $\alpha$-inhibiting therapy, support this notion, although firm conclusions should not yet be drawn from the available data. To substantiate the hypothesis that ANCA-induced activation of primed neutrophils causes vasculitis, we should therefore look for mechanisms that facilitate the pathogenic activity of ANCA in a TNF $\alpha$-independent fashion. 


\section{MPO-ANCA associated vasculitis and the complement}

\section{system}

Since ANCA-associated vasculitis is typically characterized by a paucity of immune complexes, it is generally assumed that the complement system does not play an important role in the pathogenesis of this disease. Contrary to this, several studies did find a considerable degree of immune complex depositions in skin lesions [62] as well as in renal biopsies [154] from ANCAassociated vasculitis patients. Immune complexes were also found in a rat model of MPO-ANCA associated glomerulonephritis at an early stage of disease, but not at later timepoints [47]. It is suggested from these studies that the deposition of immune complexes is an important initial trigger in the development of vascular lesions, but that they have disappeared by the time that biopsies are generally taken. To summarize, from the clinical data presented thus far, no firm conclusions can be drawn with respect to the importance of immune complex depositions and the complement system in the pathogenesis of ANCA-associated disease.



Figure 7-2: The complement system can be triggered through the classical and lectin pathway, both of which are dependent on the presence of complement factor $\mathrm{C} 4$, and through the alternative pathway, which is dependent on complement factor B. Generally, complement pathway activation leads to the formation of a C3 convertase, ultimately resulting in the generation of C5b-9 (the MAC-complex) and C3a and C5a, which are both powerful chemoattractants.

One of the advantages of the mouse model of anti-MPO IgG-induced glomerulonephritis, is that it allows the investigation of a seemingly infinite number of proteins, not only through the employment of neutralizing or depleting moAbs, but also by studying disease development in 
transgenic mice. Utilizing this approach, Xiao and colleagues recently investigated the role of the complement system (Figure 7-2) in anti-MPO IgG-induced NCGN. Much to their surprise, they found that disease development was completely inhibited in C3-depleted mice, as well as in transgenic mice deficient for complement factor C5 or factor B. Conversely, C4-deficient mice were not protected from disease induced by anti-MPO IgG [148]. It can be concluded from these experiments that alternative complement pathway activation, but not activation of the classical or lectin pathway, is somehow crucially involved in disease development.

The pivotal role of complement in anti-MPO IgG-induced NCGN, makes this system a potentially highly interesting therapeutic target. This issue is addressed in Chapter 4, in which we show the effect of a C5-inhibiting moAb (BB5.1 [149]) on the development of disease induced by the administration of anti-MPO IgG and LPS. As could be expected from the studies in complement-deficient mice, pretreatment with anti-C5 moAb completely prevented disease development upon administration of anti-MPO IgG and LPS, as measured by urinary, histopathological, and early (day 1) and late (day 7) immunohistochemical parameters. Importantly, however, we also studied the effect of anti-C5 treatment one day after disease induction, when hematuria, leukocyturia, and glomerular PMN influx are already present. This intervention resulted in a considerable decline in urinary abnormalities, as well as a more than $80 \%$ decrease in the percentage of glomeruli containing crescents and/or fibrinoid necrosis. The response to anti-C5 treatment was also reflected in several immunohistochemical parameters of glomerular inflammation. Immunofluorescence staining did not provide a clear answer with respect to the mechanism responsible for the anti-C5 effect. However, we hypothesize that properdin release from activated PMNs is involved in this process. Properdin is an important stabilizer of $\mathrm{C} 3 \mathrm{bBb}$, the alternative pathway $\mathrm{C} 3$ convertase, and therefore, its release would increase complement system activation, resulting in the generation of $\mathrm{C} 3 \mathrm{a}$ and $\mathrm{C} 5 \mathrm{a}$, both strong chemotactic and PMN-activating factors. Thus, a positive feedback loop is created, in which C3a and $\mathrm{C} 5 \mathrm{a}$ attract and activate new PMNs, resulting in the release of more properdin, so that more $\mathrm{C} 3 \mathrm{bBb}$ is stabilized (Figure 7-3) [161]. 




Figure 7-3: Proposed mechanism through which the complement system is involved in the pathogenesis of MPOANCA associated small vessel vasculitis. The $\mathrm{C} 3$ convertase $\mathrm{C} 3 \mathrm{bBb}$ is stabilized by properdin released from ANCAactivated PMNs. This induces activation of the terminal complement pathway, which entails the generation of C5a, a powerful chemoattractant and activator of PMNs.

The observation that C4-deficient mice are not protected from anti-MPO IgG-induced NCGN [148], might lead to the conclusion that immune complexes are not involved in its pathogenesis. However, it has been shown in murine heterologous nephrotoxic nephritis, a model in which disease development is generally thought to be mediated by immune complexes, that C1q- 
deficiency does not protect against disease [220]. Moreover, depending on the mouse strain used, C1q-deficiency might even lead to an aggravated response in the mouse model of heterologous [220] and accelerated [221] nephrotoxic nephritis. Obviously, there are many differences between the pathogenic mechanisms involved in nephrotoxic nephritis and anti-MPO IgG-induced glomerulonephritis, but it can be learned from those experiments that immune complex pathogenicity might under some circumstances be independent from classical pathway activation. It is conceivable that deposits of MPO/anti-MPO complexes are in part responsible for the initial glomerular sequestration of PMNs, and that the alternative complement pathway has an aggravating role [222]. Direct evidence for this mechanism is however lacking in (human) ANCA-associated vasculitis.

Our study does not only provide important new insights into the pathogenesis of anti-MPO IgGinduced NCGN, but it is also of interest from a clinical point of view. In an animal model of MPO-ANCA associated vasculitis that has many features in common with its human counterpart, we showed that C5-inhibition prohibits all pathogenic effects of anti-MPO IgG, not only when initiated prior to disease induction, but also in an intervention setup. Interestingly, C5inhibiting therapy is also already available for humans. Eculizumab is a recombinant humanized moAb that inhibits cleavage of $\mathrm{C} 5$ and thus prevents the formation of the chemotactic mediator C5a as well as the generation of CD5b-9 [223, 224]. It has recently been shown to be effective and safe in the treatment of paroxysmal nocturnal hemoglobinuria [225]. Pexelizumab, another C5-inhibiting moAb [226], has been tested mainly in patients undergoing coronary artery bypass grafts, and the safety of long-term administration of this drug is as yet undetermined. Therefore, it would be very interesting to determine whether complement pathway activation is involved in the pathogenesis of human (MPO-) ANCA associated vasculitis as well. Ultimately, such a finding could lead to a study into the effect of anti-C5 treatment in a clinical trial.

\section{Anti-MPO monoclonal antibodies in the mouse model of anti-MPO IgG-induced NCGN}

In the mouse model of anti-MPO IgG-induced NCGN, disease is induced with polyclonal antiMPO IgG, isolated from the sera from MPO-immunized $M p o^{-1-}$ mice. Consequentially, it might be consisting of several isotypes, and specific for several epitopes on the MPO-molecule. In Chapter 5, we describe the development and characterization of a panel of monoclonal antiMPO antibodies. By immunohistochemistry we show that some of the generated moAbs cross- 
react with rat MPO, and enzyme-linked immunosorbent assay (ELISA) and indirect immunofluorescence reveal that only one of the generated anti-mouse MPO moAbs is weakly cross-reactive with human MPO. The epitopes recognized by the generated moAbs are roughly clustered in three regions on the murine MPO-molecule, as determined by inhibition ELISA. Although it is not sure whether our panel of moAbs is exhaustive, our results are in accordance with studies on epitope specificity in human MPO-ANCA associated vasculitis ([173-176], reviewed in [177]).

To our surprise, none of the generated moAbs, applied in several dosages, alone or in several combinations of $\operatorname{IgG} 1, \operatorname{IgG} 2 \mathrm{a}$ and/or IgG2b clones, had the capacity to induce NCGN, either with or without LPS. The reason for this lack of pathogenicity is unknown. In our opinion, it is unlikely that the generation of new clones will yield moAbs with an epitope specificity that is importantly different from the moAbs that are already available. However, it is possible that the moAbs are only pathogenic in a specific combination of several isotypes and/or idiotypes, or that pathogenicity of a moAb relies on a specific combination of isotype and idiotype.

Whereas we could not show pathogenicity of the generated anti-MPO moAbs by themselves or in combination with a systemic proinflammatory stimulus created by the administration of LPS, two of the generated clones (one of the IgG2a and one of the IgG2b isotype) were severely pathogenic in the context of a mild immune complex glomerulonephritis, induced by the injection of sheep antibodies against the murine glomerular basement membrane (anti-GBM $\operatorname{IgG}$ ). A similar effect was already shown in two rat studies $[45,170]$, and recently, it has also been shown in mice that the presence of a polyclonal immune response against murine MPO aggravates anti-GBM glomerulonephritis [171]. However, in those studies, the anti-MPO effect was not exerted by moAbs, but by a polyclonal immune response.

The results of our study underline the pathogenicity of the anti-MPO immune response. However, it is as yet unclear as to what extent the described aggravating effects of the anti-MPO moAbs is restricted to the two tested clones. Investigation of the role of specific isotypes requires the generation of sets of clones from different isotypes, but recognizing exactly the same epitope. Similarly, to study whether the effect is epitope-specific, it would be necessary to establish sets of clones from the same isotype, recognizing different epitopes. However, after the administration of polyclonal anti-MPO $\operatorname{IgG}$, we observed that serum anti-MPO $\operatorname{IgG} 2 \mathrm{a}$ and $\operatorname{IgG} 2 \mathrm{~b}$ titers decreased more rapidly than serum anti-MPO IgG1. We therefore hypothesize that anti-MPO $\operatorname{Ig} G$ pathogenicity may predominantly be caused by antibodies of the $\operatorname{Ig} G 2 \mathrm{a}$ or $\operatorname{Ig} G 2 \mathrm{~b}$ isotype. Clearly, additional experiments are required to answer to this question. The generated panel of 
monoclonal autoantibodies might however provide interesting information concerning the role of immune complexes, complement binding, Fc-receptor involvement, and interference of the moAb with the enzymatic activity of MPO, in the pathogenesis of anti-MPO IgG-induced NCGN. Finally, although we did not yet perform experiments into this direction, it could be worthwhile to investigate the effect of an anti-MPO moAb, F(ab) fragment, or F(ab)2 fragment in combination with polyclonal anti-MPO IgG. In such an experiment, the moAb might very well aggravate disease, but it is also conceivable that the binding of non-pathogenic monoclonal antibodies (or antibody fragments) prevents the binding of pathogenic polyclonal anti-MPO IgG, and thus might lead to attenuation of disease.

\section{The role of MPO in renal inflammation}

Although similar in many ways, vasculitis patients positive for PR3-ANCA clearly differ from patients with MPO-ANCA (reviewed in [5]). This suggests that, apart from being an antigen, MPO might play an additional -functional- role in the pathogenesis of MPO-ANCA mediated disease. This is further supported by the finding that MPO-ANCA inhibit the binding of MPO to ceruloplasmin, its natural inhibitor, and thus promote the ongoing activity of MPO [28, 29]. In Chapter 6, we investigated the role of MPO in renal inflammation, by employing a mouse model of renal ischemia/reperfusion (IR) injury. By showing that MPO-deficient mice are partly protected from IR-induced renal failure, we demonstrate that this enzyme plays a hitherto unrecognized role in this model. Although it is as yet unknown what the mechanism is behind the deleterious effect of MPO in IR-induced renal inflammation, we discuss the possibility that this effect is mediated by apoptosis [23-25], activation of complement factor C5 [26, 27], and/or effects on coagulation and fibrinolysis $[213,227]$. It has been shown in vitro that MPO might be involved in CD11b/CD18 integrin-mediated PMN adherence and activation [21, 22]. In line with this, we observed a decrease in IR-induced adhesion of PMNs in $M p 0^{-/}$mice. Although the function of these cells in renal IR is as yet incompletely understood, a role for MPO in PMN adhesion in vivo might provide an attractive explanation for the protective effect of MPOdeficiency in renal IR injury and several other animal models of inflammation, and is currently being investigated. However, it remains to be investigated as to what extent the activity of MPO itself plays a role in the pathophysiology of MPO-ANCA-associated vasculitis. It might be worthwhile in this respect to see whether treatment with the MPO-inhibitor 4-aminobenzoic acid hydrazide or indomethacin [228] reduces disease in the mouse model. 


\section{Speculations on the development of ANCA-associated}

\section{vasculitis}

An important limitation of the discussed animal model is consequential to the artificial way in which the anti-MPO immune response is elicited: they are not suitable to study the origin of the autoimmune response to MPO. In this respect, infection [69-72, 229] and other environmental factors such as silica ([230-233], reviewed in [234]) have long been suggested to play a role in the induction of the ANCA response. This might also be the case for MPO-ANCA, although there is little support for this view from clinical as well as experimental studies. In addition, as discussed in the introduction of this thesis, future studies will have to establish the value of the theory of autoantigen complementarity [32] as a mechanism contributing to the initiation of the anti-PR3, and possibly the anti-MPO autoimmune response.

It is possible that any factor responsible for the initiation of an anti-MPO immune response, is also responsible for its perpetuation. However, if such a trigger is temporary, as would probably be the case when disease onset is consequential to an infection, as suggested by some researchers [32, 69-72], an explanation should be found for the continuation of ANCA-production after termination of the triggering factor. In this respect, it might be interesting to note that in preliminary experiments in our mouse model, we found increased numbers of periglomerular class II major histocompatibility complex and CD11c-positive dendritic cells (DCs). This is in line with observations by Krüger and colleagues, who identified similar depositions of CD11cpositive cells in a mouse model of nephrotoxic nephritis, and were able to show that these cells could act as dendritic cells in vitro [235].

Although far from proven, the presence of functionally active dendritic cells in the glomerular infiltrates would make it defendable to propose a hypothesis in which the cause of ongoing production of ANCA lies within the inflamed glomeruli themselves. Support for this mechanism is provided by a study showing that the administration of apoptotic PMNs can lead to the development of ANCA [54]. In addition, ANCA have been shown to disturb normal apoptosis of TNF $\alpha$-primed PMNs, leading to a decreased phagocytosis by macrophages [236]. This might cause secondary necrosis, which is a highly inflammatory event, potentially leading to the maturation and activation of dendritic cells [237]. Alternatively, it has been shown by Harper and colleagues that the opsonization with ANCA of apoptotic PMNs actually increases their uptake by macrophages, but that this occurs in a proinflammatory fashion, leading to an increased release of IL-1 and IL-8 [17]. Irrespective of the cause of inflammation however, it is conceivable 
that in a phlogistic milieu, characterized by the presence of activated, apoptotic and/or necrotic PMNs, DCs, and T-cells, DCs might process MPO in such a way, that it results in the activation of T-cells and the generation of autoimmunity. In this respect, it has already been shown that mice injected with DCs that are loaded in vitro with the cardiac autoantigen MYHC- $\alpha$, develop autoimmune myocarditis if the DCs were appropriately activated with LPS and a costimulatory anti-CD40 antibody [238]. Moreover, a similar mechanism of activation of T-cells by lesional DCs has already been suggested to play a role in murine autoimmune diabetes [239] and human autoimmune thyroiditis [240]. Also in ANCA-associated disease, generation of autoantibodies at the site of inflammation has been proposed previously [100, 241], and is supported by the presence of ANCA in broncho-alveolar lavage fluid of patients [242]. However, it remains a challenge to prove a role for this phenomenon in vivo. A better understanding of the pathophysiology behind the perpetuation of ANCA production has not only gained priority after the discovery of MPO-ANCA pathogenicity in rodent models [43, 49], but those models also offer an exiting opportunity to investigate this issue in an in vivo setting.

\section{Conclusion}

The development of the mouse model of anti-MPO IgG-induced vasculitis has led to a series of studies that largely confirmed the hypothesis derived from in vitro studies, that MPO-ANCA are pathogenetically involved in MPO-ANCA associated vasculitis through their capacity to activate TNF $\alpha$-primed PMNs. Unexpectedly however, the complement system has recently been shown to be crucially involved in disease pathogenesis. Additional studies will have to reveal the responsible mechanism. Nevertheless, if the results obtained in the mouse model also apply to human (MPO-) ANCA-associated disease, this does not only call for a modification of the current hypothesis, but also implies an exciting opportunity for novel therapeutic strategies. The therapeutic value of a panel of murine MPO-specific moAbs, as well as mounting data on the versatile functions of MPO in vivo, remain to be established. Finally, although considerable progress has been made in this field as well, it is as yet a challenge to elucidate the mechanisms that are responsible for the derangement of the immune system underlying the formation of an autoimmune response. 


\section{References}

1. Weyand CM. Vasculitis: A dialogue between the artery and the immune system. In: Hoffman GS, Weyand CM (eds). Inflammatory Diseases of Blood Vessels. Marcel Dekker, Inc.: New York, 2002, pp 1-12.

2. Jennette JC, Falk RJ, Andrassy K, Bacon PA, et al. Nomenclature of systemic vasculitides. Proposal of an international consensus conference. Arthritis Rheum 1994; 37: 187-192.

3. Jennette JC, Falk RJ. Small-vessel vasculitis. N Engl J Med 1997; 337: 1512-1523.

4. Bartunkova J, Tesar V, Sediva A. Diagnostic and pathogenetic role of antineutrophil cytoplasmic autoantibodies. Clin Immunol 2003; 106: 73-82.

5. Franssen CF, Stegeman CA, Kallenberg CG, Gans RO, et al. Antiproteinase 3- and antimyeloperoxidaseassociated vasculitis. Kidney Int 2000; 57: 2195-2206.

6. Jennette JC. Rapidly progressive crescentic glomerulonephritis. Kidney Int 2003; 63: 1164-1177.

7. Cohen Tervaert JW, Huitema MG, Hene RJ, Sluiter WJ, et al. Prevention of relapses in Wegener's granulomatosis by treatment based on antineutrophil cytoplasmic antibody titre. Lancet 1990; 336: 709-711.

8. Jayne D, Rasmussen N, Andrassy K, Bacon P, et al. A Randomized Trial of Maintenance Therapy for Vasculitis Associated with Antineutrophil Cytoplasmic Autoantibodies. N Engl J Med 2003; 349: 36-44.

9. Falk RJ, Terrell RS, Charles LA, Jennette JC. Anti-neutrophil cytoplasmic autoantibodies induce neutrophils to degranulate and produce oxygen radicals in vitro. Proc Natl Acad Sci U S A 1990; 87: 4115-4119.

10. Franssen CF, Huitema MG, Muller Kobold AC, Oost-Kort WW, et al. In vitro neutrophil activation by antibodies to proteinase 3 and myeloperoxidase from patients with crescentic glomerulonephritis. J Am Soc Nephrol 1999; 10: 1506-1515.

11. Hess C, Sadallah S, Schifferli JA. Induction of neutrophil responsiveness to myeloperoxidase antibodies by their exposure to supernatant of degranulated autologous neutrophils. Blood 2000; 96: 2822-2827.

12. Kettritz R, Schreiber A, Luft FC, Haller H. Role of mitogen-activated protein kinases in activation of human neutrophils by antineutrophil cytoplasmic antibodies. J Am Soc Nephrol 2001; 12: 37-46.

13. Rarok AA, Limburg PC, Kallenberg CG. Neutrophil-activating potential of antineutrophil cytoplasm autoantibodies. J Lenkoc Biol 2003; 74: 3-15.

14. Hewins P, Morgan MD, Holden N, Neil D, et al. IL-18 is upregulated in the kidney and primes neutrophil responsiveness in ANCA-associated vasculitis. Kidney Int 2006; 69: 605-615.

15. Ewert BH, Jennette JC, Falk RJ. Anti-myeloperoxidase antibodies stimulate neutrophils to damage human endothelial cells. Kidney Int 1992; 41: 375-383.

16. Radford DJ, Luu NT, Hewins P, Nash GB, et al. Antineutrophil cytoplasmic antibodies stabilize adhesion and promote migration of flowing neutrophils on endothelial cells. Arthritis Rheum 2001; 44: 2851-2861.

17. Harper L, Cockwell P, Adu D, Savage CO. Neutrophil priming and apoptosis in anti-neutrophil cytoplasmic autoantibody-associated vasculitis. Kidney Int 2001; 59: 1729-1738.

18. Heeringa P, Huugen D, Tervaert JW. Anti-neutrophil cytoplasmic autoantibodies and leukocyte-endothelial interactions: a sticky connection? Trends Immunol 2005; 26: 561-564.

19. Malle E, Buch T, Grone HJ. Myeloperoxidase in kidney disease. Kidney Int 2003; 64: 1956-1967.

20. Klebanoff SJ. Myeloperoxidase: friend and foe. J Lenkoc Biol 2005; 77: 598-625.

21. Johansson MW, Patarroyo M, Oberg F, Siegbahn A, et al. Myeloperoxidase mediates cell adhesion via the alpha M beta 2 integrin (Mac-1, CD11b/CD18). J Cell Sci 1997; 110 ( Pt 9): 1133-1139.

22. Lau D, Mollnau H, Eiserich JP, Freeman BA, et al. Myeloperoxidase mediates neutrophil activation by association with CD11b/CD18 integrins. Proc Natl Acad Sci U S A 2005; 102: 431-436.

23. Wagner BA, Buettner GR, Oberley LW, Darby CJ, et al. Myeloperoxidase is involved in H2O2-induced apoptosis of HL-60 human leukemia cells. J Biol Chem 2000; 275: 22461-22469.

24. Tsurubuchi T, Aratani Y, Maeda N, Koyama H. Retardation of early-onset PMA-induced apoptosis in mouse neutrophils deficient in myeloperoxidase. J Leukoc Biol 2001; 70: 52-58.

25. Myzak MC, Carr AC. Myeloperoxidase-dependent caspase-3 activation and apoptosis in HL-60 cells: protection by the antioxidants ascorbate and (dihydro)lipoic acid. Redox Rep 2002; 7: 47-53.

26. Vogt W, Hesse D. Oxidants generated by the myeloperoxidase-halide system activate the fifth component of human complement, C5. Immunobiology 1994; 192: 1-9.

27. Vogt W. Complement activation by myeloperoxidase products released from stimulated human polymorphonuclear leukocytes. Immunobiology 1996; 195: 334-346.

28. Segelmark M, Persson B, Hellmark T, Wieslander J. Binding and inhibition of myeloperoxidase (MPO): a major function of ceruloplasmin? Clin Exp Immunol 1997; 108: 167-174.

29. Griffin SV, Chapman PT, Lianos EA, Lockwood CM. The inhibition of myeloperoxidase by ceruloplasmin can be reversed by anti-myeloperoxidase antibodies. Kidney Int 1999; 55: 917-925. 
30. Brons RH, Kallenberg CG, Cohen Tervaert JW. Are antineutrophil cytoplasmic antibody-associated vasculitides pauci-immune? Rheum Dis Clin North Am 2001; 27: 833-848.

31. Shoenfeld Y. Idiotypic induction of autoimmunity: a new aspect of the idiotypic network. Faseb J 1994; 8: 1296-1301.

32. Pendergraft WF, Preston GA, Shah RR, Tropsha A, et al. Autoimmunity is triggered by cPR-3(105-201), a protein complementary to human autoantigen proteinase-3. Nat Med 2004; 10: 72-79.

33. Popa ER, Stegeman CA, Kallenberg CG, Tervaert JW. Staphylococcus aureus and Wegener's granulomatosis. Arthritis Res 2002; 4: 77-79.

34. Mathieson PW, Thiru S, Oliveira DB. Mercuric chloride-treated brown Norway rats develop widespread tissue injury including necrotizing vasculitis. Lab Invest 1992; 67: 121-129.

35. Heeringa P, Brouwer E, Cohen Tervaert JW, Weening JJ, et al. Animal models of anti-neutrophil cytoplasmic antibody associated vasculitis. Kidney Int 1998; 53: 253-263.

36. Kiely PD, Pecht I, Oliveira DB. Mercuric chloride-induced vasculitis in the Brown Norway rat: alpha beta T cell-dependent and -independent phases: role of the mast cell. J Immunol 1997; 159: 5100-5106.

37. Macphee IA, Turner DR, Yagita H, Oliveira DB. The Th2-response in mercuric chloride-induced autoimmunity requires continuing costimulation via CD28. Clin Exp Immunol 2002; 129: 405-410.

38. Brouwer E, Stegeman CA, Huitema MG, Limburg PC, et al. T cell reactivity to proteinase 3 and myeloperoxidase in patients with Wegener's granulomatosis (WG). Clin Exp Immunol 1994; 98: 448-453.

39. King WJ, Brooks CJ, Holder R, Hughes P, et al. T lymphocyte responses to anti-neutrophil cytoplasmic autoantibody (ANCA) antigens are present in patients with ANCA-associated systemic vasculitis and persist during disease remission. Clin Exp Immunol 1998; 112: 539-546.

40. Clayton AR, Savage CO. What you should know about PR3-ANCA. Evidence for the role of T cells in the pathogenesis of systemic vasculitis. Arthritis Res 2000; 2: 260-262.

41. Popa ER, Franssen CF, Limburg PC, Huitema MG, et al. In vitro cytokine production and proliferation of $T$ cells from patients with anti-proteinase 3 - and antimyeloperoxidase-associated vasculitis, in response to proteinase 3 and myeloperoxidase. Arthritis Rheum 2002; 46: 1894-1904.

42. Clayton AR, Savage CO. Production of antineutrophil cytoplasm antibodies derived from circulating B cells in patients with systemic vasculitis. Clin Exp Immunol 2003; 132: 174-179.

43. Xiao H, Heeringa P, Hu P, Liu Z, et al. Antineutrophil cytoplasmic autoantibodies specific for myeloperoxidase cause glomerulonephritis and vasculitis in mice. J Clin Invest 2002; 110: 955-963.

44. Wu Z, Turner DR, Oliveira DB. Antioxidants inhibit mercuric chloride-induced early vasculitis. Int Immunol 2002; 14: 267-273.

45. Heeringa P, Brouwer E, Klok PA, Huitema MG, et al. Autoantibodies to myeloperoxidase aggravate mild anti-glomerular-basement-membrane-mediated glomerular injury in the rat. Am J Pathol 1996; 149: 16951706.

46. Heeringa P, Foucher P, Klok PA, Huitema MG, et al. Systemic injection of products of activated neutrophils and $\mathrm{H} 2 \mathrm{O} 2$ in myeloperoxidase-immunized rats leads to necrotizing vasculitis in the lungs and gut. Am J Pathol 1997; 151: 131-140.

47. Brouwer E, Huitema MG, Klok PA, de Weerd H, et al. Antimyeloperoxidase-associated proliferative glomerulonephritis: an animal model. J Exp Med 1993; 177: 905-914.

48. Yang JJ, Jennette JC, Falk RJ. Immune complex glomerulonephritis is induced in rats immunized with heterologous myeloperoxidase. Clin Exp Immunol 1994; 97: 466-473.

49. Little MA, Smyth CL, Yadav R, Ambrose L, et al. Antineutrophil cytoplasm antibodies directed against myeloperoxidase augment leukocyte-microvascular interactions in vivo. Blood 2005; 106: 2050-2058.

50. Savige J, Nassis L, Cooper T, Paspaliaris B, et al. Antineutrophil cytoplasmic antibody (ANCA)-associated systemic vasculitis after immunisation with bacterial proteins. Clin Exp Rheumatol 2002; 20: 783-789.

51. Blank M, Tomer Y, Stein M, Kopolovic J, et al. Immunization with anti-neutrophil cytoplasmic antibody (ANCA) induces the production of mouse ANCA and perivascular lymphocyte infiltration. Clin Exp Immunol 1995; 102: 120-130.

52. Jenne DE, Frohlich L, Hummel AM, Specks U. Cloning and functional expression of the murine homologue of proteinase 3: implications for the design of murine models of vasculitis. FEBS Lett 1997; 408: $187-190$.

53. Rauova L, Gilburd B, Zurgil N, Blank M, et al. Induction of biologically active antineutrophil cytoplasmic antibodies by immunization with human apoptotic polymorphonuclear leukocytes. Clin Immunol 2002; 103: 69-78.

54. Patry YC, Trewick DC, Gregoire M, Audrain MA, et al. Rats injected with syngenic rat apoptotic neutrophils develop antineutrophil cytoplasmic antibodies. J Am Soc Nephrol 2001; 12: 1764-1768.

55. Clayton AR, Prue RL, Harper L, Drayson MT, et al. Dendritic cell uptake of human apoptotic and necrotic neutrophils inhibits CD40, CD80, and CD86 expression and reduces allogeneic T cell responses: Relevance to systemic vasculitis. Arthritis Rheum 2003; 48: 2362-2374. 
56. Kinjoh K, Kyogoku M, Good RA. Genetic selection for crescent formation yields mouse strain with rapidly progressive glomerulonephritis and small vessel vasculitis. Proc Natl Acad Sci U S A 1993; 90: 3413-3417.

57. Andrews BS, Eisenberg RA, Theofilopoulos AN, Izui S, et al. Spontaneous murine lupus-like syndromes. Clinical and immunopathological manifestations in several strains. J Exp Med 1978; 148: 1198-1215.

58. Harper JM, Thiru S, Lockwood CM, Cooke A. Myeloperoxidase autoantibodies distinguish vasculitis mediated by anti-neutrophil cytoplasm antibodies from immune complex disease in MRL/Mp-lpr/lpr mice: a spontaneous model for human microscopic angiitis. Eur J Immunol 1998; 28: 2217-2226.

59. Harper JM, Healey DG, Thiru S, Gordon C, et al. Factors involved in the pathogenesis of neutrophilic vasculitis in MRL/Mp-lpr/lpr mice: a model for human microscopic angiitis. Autoimmunity 1999; 31: 133145.

60. Neumann I, Birck R, Newman M, Schnulle P, et al. SCG/Kinjoh mice: A model of ANCA-associated crescentic glomerulonephritis with immune deposits. Kidney Int 2003; 64: 140-148.

61. Aratani Y, Koyama H, Nyui S, Suzuki K, et al. Severe impairment in early host defense against Candida albicans in mice deficient in myeloperoxidase. Infect Immun 1999; 67: 1828-1836.

62. Brons RH, de Jong MC, de Boer NK, Stegeman CA, et al. Detection of immune deposits in skin lesions of patients with Wegener's granulomatosis. Ann Rheum Dis 2001; 60: 1097-1102.

63. Bansal PJ, Tobin MC. Neonatal microscopic polyangiitis secondary to transfer of maternal myeloperoxidase-antineutrophil cytoplasmic antibody resulting in neonatal pulmonary hemorrhage and renal involvement. Ann Allergy Asthma Immunol 2004; 93: 398-401.

64. Pfister H, Ollert M, Frohlich LF, Quintanilla-Martinez L, et al. Antineutrophil cytoplasmic autoantibodies against the murine homolog of proteinase 3 (Wegener autoantigen) are pathogenic in vivo. Blood 2004; 104: 1411-1418.

65. Kallenberg CG, Brouwer E, Weening JJ, Tervaert JW. Anti-neutrophil cytoplasmic antibodies: current diagnostic and pathophysiological potential. Kidney Int 1994; 46: 1-15.

66. Han WK, Choi HK, Roth RM, McCluskey RT, et al. Serial ANCA titers: Useful tool for prevention of relapses in ANCA-associated vasculitis. Kidney Int 2003; 63: 1079-1085.

67. Boomsma MM, Stegeman CA, van der Leij MJ, Oost W, et al. Prediction of relapses in Wegener's granulomatosis by measurement of antineutrophil cytoplasmic antibody levels: a prospective study. Arthritis Rheum 2000; 43: 2025-2033.

68. Tervaert JW, van der Woude FJ, Fauci AS, Ambrus JL, et al. Association between active Wegener's granulomatosis and anticytoplasmic antibodies. Arch Intern Med 1989; 149: 2461-2465.

69. Falk RJ, Hogan S, Carey TS, Jennette JC. Clinical course of anti-neutrophil cytoplasmic autoantibodyassociated glomerulonephritis and systemic vasculitis. The Glomerular Disease Collaborative Network. Ann Intern Med 1990; 113: 656-663.

70. Pinching AJ, Rees AJ, Pussell BA, Lockwood CM, et al. Relapses in Wegener's granulomatosis: the role of infection. BrMed J 1980; 281: 836-838.

71. Arimura Y, Minoshima S, Kamiya Y, Tanaka U, et al. Serum myeloperoxidase and serum cytokines in antimyeloperoxidase antibody-associated glomerulonephritis. Clin Nephrol 1993; 40: 256-264.

72. Capizzi SA, Specks U. Does infection play a role in the pathogenesis of pulmonary vasculitis? Semin Respir Infect 2003; 18: 17-22.

73. Reumaux D, Vossebeld PJ, Roos D, Verhoeven AJ. Effect of tumor necrosis factor-induced integrin activation on Fc gamma receptor II-mediated signal transduction: relevance for activation of neutrophils by anti-proteinase 3 or anti-myeloperoxidase antibodies. Blood 1995; 86: 3189-3195.

74. Dentener MA, Greve JW, Maessen JG, Buurman WA. Role of tumour necrosis factor in the enhanced sensitivity of mice to endotoxin after exposure to lead. Immunopharmacol Immunotoxicol 1989; 11: 321-334.

75. Sheehan KC, Ruddle NH, Schreiber RD. Generation and characterization of hamster monoclonal antibodies that neutralize murine tumor necrosis factors. J Immunol 1989; 142: 3884-3893.

76. Vernooy JH, Dentener MA, van Suylen RJ, Buurman WA, et al. Intratracheal instillation of lipopolysaccharide in mice induces apoptosis in bronchial epithelial cells: no role for tumor necrosis factoralpha and infiltrating neutrophils. Am J Respir Cell Mol Biol 2001; 24: 569-576.

77. Akashi S, Shimazu R, Ogata H, Nagai Y, et al. Cutting edge: cell surface expression and lipopolysaccharide signaling via the toll-like receptor 4-MD-2 complex on mouse peritoneal macrophages. J Immunol 2000; 164: 3471-3475.

78. Lopez AF, Strath M, Sanderson CJ. Differentiation antigens on mouse eosinophils and neutrophils identified by monoclonal antibodies. BrJ Haematol 1984; 57: 489-494.

79. Masaki T, Chow F, Nikolic-Paterson DJ, Atkins RC, et al. Heterogeneity of antigen expression explains controversy over glomerular macrophage accumulation in mouse glomerulonephritis. Nephrol Dial Transplant 2003; 18: 178-181. 
80. Pick E, Mizel D. Rapid microassays for the measurement of superoxide and hydrogen peroxide production by macrophages in culture using an automatic enzyme immunoassay reader. J Immunol Methods 1981; 46: 211-226.

81. Ewert BH, Becker ME, Jennette JC, Falk RJ. Antimyeloperoxidase antibodies induce neutrophil adherence to cultured human endothelial cells. Ren Fail 1995; 17: 125-133.

82. Reumaux D, De Boer M, Meijer AB, Duthilleul P, et al. Expression of myeloperoxidase (MPO) by neutrophils is necessary for their activation by anti-neutrophil cytoplasm autoantibodies (ANCA) against MPO.J Leukoc Biol 2003; 73: 841-849.

83. Lan HY. Therapeutic effects of cytokine blockade in glomerulonephritis. Nephrol Dial Transplant 1998; 13: 79 .

84. Vassalli P. The pathophysiology of tumor necrosis factors. Annu Rev Immunol 1992; 10: 411-452.

85. Le Hir M, Haas C, Marino M, Ryffel B. Prevention of crescentic glomerulonephritis induced by antiglomerular membrane antibody in tumor necrosis factor-deficient mice. Lab Invest 1998; 78: 1625-1631.

86. Karkar AM, Smith J, Pusey CD. Prevention and treatment of experimental crescentic glomerulonephritis by blocking tumour necrosis factor-alpha. Nephrol Dial Transplant 2001; 16: 518-524.

87. Booth A, Harper L, Hammad T, Bacon P, et al. Prospective study of TNFalpha blockade with infliximab in anti-neutrophil cytoplasmic antibody-associated systemic vasculitis. J Am Soc Nephrol 2004; 15: 717-721.

88. Slot MC, Cohen Tervaert JW, Franssen CF, Stegeman CA. Renal survival and prognostic factors in patients with PR3-ANCA associated vasculitis with renal involvement. Kidney Int 2003; 63: 670-677.

89. Bathon JM, Martin RW, Fleischmann RM, Tesser JR, et al. A comparison of etanercept and methotrexate in patients with early rheumatoid arthritis. N Engl J Med 2000; 343: 1586-1593.

90. Lipsky PE, van der Heijde DM, St Clair EW, Furst DE, et al. Infliximab and methotrexate in the treatment of rheumatoid arthritis. Anti-Tumor Necrosis Factor Trial in Rheumatoid Arthritis with Concomitant Therapy Study Group. N Engl J Med 2000; 343: 1594-1602.

91. St Clair EW, van der Heijde DM, Smolen JS, Maini RN, et al. Combination of infliximab and methotrexate therapy for early rheumatoid arthritis: a randomized, controlled trial. Arthritis Rheum 2004; 50: 3432-3443.

92. Targan SR, Hanauer SB, van Deventer SJ, Mayer L, et al. A short-term study of chimeric monoclonal antibody cA2 to tumor necrosis factor alpha for Crohn's disease. Crohn's Disease cA2 Study Group. $N$ Engl J Med 1997; 337: 1029-1035.

93. Braun J, Brandt J, Listing J, Zink A, et al. Treatment of active ankylosing spondylitis with infliximab: a randomised controlled multicentre trial. Lancet 2002; 359: 1187-1193.

94. Gorman JD, Sack KE, Davis JC, Jr. Treatment of ankylosing spondylitis by inhibition of tumor necrosis factor alpha. N Engl J Med 2002; 346: 1349-1356.

95. Hoffman GS, Merkel PA, Brasington RD, Lenschow DJ, et al. Anti-tumor necrosis factor therapy in patients with difficult to treat Takayasu arteritis. Arthritis Rheum 2004; 50: 2296-2304.

96. Ohno S, Nakamura S, Hori S, Shimakawa M, et al. Efficacy, safety, and pharmacokinetics of multiple administration of infliximab in Behcet's disease with refractory uveoretinitis. J Rheumatol 2004; 31: 1362 1368.

97. Melikoglu M, Fresko I, Mat C, Ozyazgan Y, et al. Short-term trial of etanercept in Behcet's disease: a double blind, placebo controlled study. J Rheumatol 2005; 32: 98-105.

98. Tugal-Tutkun I, Mudun A, Urgancioglu M, Kamali S, et al. Efficacy of infliximab in the treatment of uveitis that is resistant to treatment with the combination of azathioprine, cyclosporine, and corticosteroids in Behcet's disease: an open-label trial. Arthritis Rheum 2005; 52: 2478-2484.

99. Porges AJ, Redecha PB, Kimberly WT, Csernok E, et al. Anti-neutrophil cytoplasmic antibodies engage and activate human neutrophils via Fc gamma RIIa. J Immunol 1994; 153: 1271-1280.

100. Harper L, Radford D, Plant T, Drayson M, et al. IgG from myeloperoxidase-antineutrophil cytoplasmic antibody-positive patients stimulates greater activation of primed neutrophils than IgG from proteinase 3antineutrophil cytosplasmic antibody-positive patients. Arthritis Rheum 2001; 44: 921-930.

101. Condliffe AM, Chilvers ER, Haslett C, Dransfield I. Priming differentially regulates neutrophil adhesion molecule expression/function. Immunology 1996; 89: 105-111.

102. Savage CO, Pottinger BE, Gaskin G, Pusey CD, et al. Autoantibodies developing to myeloperoxidase and proteinase 3 in systemic vasculitis stimulate neutrophil cytotoxicity toward cultured endothelial cells. $A m J$ Pathol 1992; 141: 335-342.

103. Khan SB, Cook HT, Bhangal G, Smith J, et al. Antibody blockade of TNF-alpha reduces inflammation and scarring in experimental crescentic glomerulonephritis. Kidney Int 2005; 67: 1812-1820.

104. Ishida-Okawara A, Ito-Ihara T, Muso E, Ono T, et al. Neutrophil contribution to the crescentic glomerulonephritis in SCG/Kj mice. Nephrol Dial Transplant 2004; 19: 1708-1715.

105. Huugen D, Xiao H, van Esch A, Falk RJ, et al. Aggravation of Anti-Myeloperoxidase Antibody-Induced Glomerulonephritis by Bacterial Lipopolysaccharide: Role of Tumor Necrosis Factor-\{alpha\}. Am J Pathol 2005; 167: 47-58. 
106. Little MA, Bhangal G, Smyth CL, Nakada MT, et al. Therapeutic Effect of Anti-TNF-\{alpha\} Antibodies in an Experimental Model of Anti-Neutrophil Cytoplasm Antibody-Associated Systemic Vasculitis. J Am Soc Nephrol 2006; 17: 160-169.

107. Hochberg MC, Tracy JK, Hawkins-Holt M, Flores RH. Comparison of the efficacy of the tumour necrosis factor alpha blocking agents adalimumab, etanercept, and infliximab when added to methotrexate in patients with active rheumatoid arthritis. Ann Rheum Dis 2003; 62 Suppl 2: ii13-16.

108. Sandborn WJ, Hanauer SB, Katz S, Safdi M, et al. Etanercept for active Crohn's disease: a randomized, double-blind, placebo-controlled trial. Gastroenterology 2001; 121: 1088-1094.

109. Rau R. Adalimumab (a fully human anti-tumour necrosis factor alpha monoclonal antibody) in the treatment of active rheumatoid arthritis: the initial results of five trials. Ann Rheum Dis 2002; 61 Suppl 2: ii70-73.

110. Wick MC, Ernestam S, Lindblad S, Bratt J, et al. Adalimumab (Humira) restores clinical response in patients with secondary loss of efficacy from infliximab (Remicade) or etanercept (Enbrel): results from the STURE registry at Karolinska University Hospital. Scand J Rheumatol 2005; 34: 353-358.

111. Papadakis KA, Shaye OA, Vasiliauskas EA, Ippoliti A, et al. Safety and efficacy of adalimumab (D2E7) in Crohn's disease patients with an attenuated response to infliximab. Am J Gastroenterol 2005; 100: 75-79.

112. Noronha IL, Kruger C, Andrassy K, Ritz E, et al. In situ production of TNF-alpha, IL-1 beta and IL-2R in ANCA-positive glomerulonephritis. Kidney Int 1993; 43: 682-692.

113. Tesar V, Masek Z, Rychlik I, Merta M, et al. Cytokines and adhesion molecules in renal vasculitis and lupus nephritis. Nephrol Dial Transplant 1998; 13: 1662-1667.

114. Ito-Ihara T, Ono T, Nogaki F, Suyama K, et al. Clinical efficacy of intravenous immunoglobulin for patients with MPO-ANCA-associated rapidly progressive glomerulonephritis. Nephron Clin Pract 2006; 102: c35-42.

115. Feldmann M, Maini RN. Lasker Clinical Medical Research Award. TNF defined as a therapeutic target for rheumatoid arthritis and other autoimmune diseases. Nat Med 2003; 9: 1245-1250.

116. Lamprecht P, Voswinkel J, Lilienthal T, Nolle B, et al. Effectiveness of TNF-alpha blockade with infliximab in refractory Wegener's granulomatosis. Rheumatology (Oxford) 2002; 41: 1303-1307.

117. Kleinert J, Lorenz M, Kostler W, Horl W, et al. Refractory Wegener's granulomatosis responds to tumor necrosis factor blockade. Wien Klin Wochenschr 2004; 116: 334-338.

118. Luqmani RA, Bacon PA, Moots RJ, Janssen BA, et al. Birmingham Vasculitis Activity Score (BVAS) in systemic necrotizing vasculitis. Qjm 1994; 87: 671-678.

119. Rutgeerts P, D'Haens G, Targan S, Vasiliauskas E, et al. Efficacy and safety of retreatment with anti-tumor necrosis factor antibody (infliximab) to maintain remission in Crohn's disease. Gastroenterology 1999; 117: 761-769.

120. Present DH, Rutgeerts P, Targan S, Hanauer SB, et al. Infliximab for the treatment of fistulas in patients with Crohn's disease. N Engl J Med 1999; 340: 1398-1405.

121. Hanauer SB, Feagan BG, Lichtenstein GR, Mayer LF, et al. Maintenance infliximab for Crohn's disease: the ACCENT I randomised trial. Lancet 2002; 359: 1541-1549.

122. Vermeire S, Noman M, Van Assche G, Baert F, et al. Autoimmunity associated with anti-tumor necrosis factor alpha treatment in Crohn's disease: a prospective cohort study. Gastroenterology 2003; 125: 32-39.

123. Booth AD, Jayne DR, Kharbanda RK, McEniery CM, et al. Infliximab Improves Endothelial Dysfunction in Systemic Vasculitis. A Model of Vascular Inflammation. Circulation 2004; 109: 1718-1723.

124. Bartolucci P, Ramanoelina J, Cohen P, Mahr A, et al. Efficacy of the anti-TNF-alpha antibody infliximab against refractory systemic vasculitides: an open pilot study on 10 patients. Rheumatology (Oxford) 2002; 41: 1126-1132.

125. Zaenker M, Arbach O, Helmchen U, Glorius P, et al. Crescentic glomerulonephritis associated with myeloperoxidase-antineutrophil-cytoplasmic antibodies: first report on the efficacy of primary anti-TNFalpha treatment. Int J Tissue React 2004; 26: 85-92.

126. Etanercept plus standard therapy for Wegener's granulomatosis. N Engl J Med 2005; 352: 351-361.

127. Scallon B, Cai A, Solowski N, Rosenberg A, et al. Binding and functional comparisons of two types of tumor necrosis factor antagonists. J Pharmacol Exp Ther 2002; 301: 418-426.

128. Mitoma $\mathrm{H}$, Horiuchi $\mathrm{T}$, Hatta $\mathrm{N}$, Tsukamoto $\mathrm{H}$, et al. Infliximab induces potent anti-inflammatory responses by outside-to-inside signals through transmembrane TNF-alpha. Gastroenterology 2005; 128: 376392.

129. Van den Brande JM, Braat H, van den Brink GR, Versteeg HH, et al. Infliximab but not etanercept induces apoptosis in lamina propria T-lymphocytes from patients with Crohn's disease. Gastroenterology 2003; 124: 1774-1785.

130. Agnholt J, Dahlerup JF, Kaltoft K. The effect of etanercept and infliximab on the production of tumour necrosis factor alpha, interferon-gamma and GM-CSF in in vivo activated intestinal T lymphocyte cultures. Cytokine 2003; 23: 76-85. 
131. Kirchner S, Holler E, Haffner S, Andreesen R, et al. Effect of different tumor necrosis factor (TNF) reactive agents on reverse signaling of membrane integrated TNF in monocytes. Cytokine 2004; 28: 67-74.

132. Keane J, Gershon S, Wise RP, Mirabile-Levens E, et al. Tuberculosis associated with infliximab, a tumor necrosis factor alpha-neutralizing agent. N Engl J Med 2001; 345: 1098-1104.

133. Balkwill F. Tumor necrosis factor or tumor promoting factor? Cytokine Growth Factor Rev 2002; 13: $135-141$.

134. Wolfe F, Michaud K. Lymphoma in rheumatoid arthritis: the effect of methotrexate and anti-tumor necrosis factor therapy in 18,572 patients. Arthritis Rheum 2004; 50: 1740-1751.

135. Brown SL, Greene MH, Gershon SK, Edwards ET, et al. Tumor necrosis factor antagonist therapy and lymphoma development: twenty-six cases reported to the Food and Drug Administration. Arthritis Rheum 2002; 46: 3151-3158.

136. Geborek P, Bladstrom A, Turesson C, Gulfe A, et al. Tumour necrosis factor blockers do not increase overall tumour risk in patients with rheumatoid arthritis, but may be associated with an increased risk of lymphomas. Ann Rheum Dis 2005; 64: 699-703.

137. Merkel PA, Lo GH, Holbrook JT, Tibbs AK, et al. Brief communication: high incidence of venous thrombotic events among patients with Wegener granulomatosis: the Wegener's Clinical Occurrence of Thrombosis (WeCLOT) Study. Ann Intern Med 2005; 142: 620-626.

138. Joseph L, Fink LM, Hauer-Jensen M. Cytokines in coagulation and thrombosis: a preclinical and clinical review. Blood Coagul Fibrinolysis 2002; 13: 105-116.

139. van der Poll T, Levi M, van Deventer SJ, ten Cate H, et al. Differential effects of anti-tumor necrosis factor monoclonal antibodies on systemic inflammatory responses in experimental endotoxemia in chimpanzees. Blood 1994; 83: 446-451.

140. Jonsdottir T, Forslid J, van Vollenhoven A, Harju A, et al. Treatment with tumour necrosis factor alpha antagonists in patients with rheumatoid arthritis induces anticardiolipin antibodies. Ann Rheum Dis 2004; 63: 1075-1078.

141. Atzeni F, Turiel M, Capsoni F, Doria A, et al. Autoimmunity and Anti-TNF-\{alpha\} Agents. Ann N Y Acad Sci 2005; 1051: 559-569.

142. Brion PH, Mittal-Henkle A, Kalunian KC. Autoimmune skin rashes associated with etanercept for rheumatoid arthritis. Ann Intern Med 1999; 131: 634.

143. Charles PJ, Smeenk RJ, De Jong J, Feldmann M, et al. Assessment of antibodies to double-stranded DNA induced in rheumatoid arthritis patients following treatment with infliximab, a monoclonal antibody to tumor necrosis factor alpha: findings in open-label and randomized placebo-controlled trials. Arthritis Rheum 2000; 43: 2383-2390.

144. Shakoor N, Michalska M, Harris CA, Block JA. Drug-induced systemic lupus erythematosus associated with etanercept therapy. Lancet 2002; 359: 579-580.

145. Ferraro-Peyret C, Coury F, Tebib JG, Bienvenu J, et al. Infliximab therapy in rheumatoid arthritis and ankylosing spondylitis-induced specific antinuclear and antiphospholipid autoantibodies without autoimmune clinical manifestations: a two-year prospective study. Arthritis Res Ther 2004; 6: R535-543.

146. Christopher-Stine L, Wigley F. Tumor necrosis factor-alpha antagonists induce lupus-like syndrome in patients with scleroderma overlap/mixed connective tissue disease. J Rheumatol 2003; 30: 2725-2727.

147. Xiao H, Heeringa P, Liu Z, Huugen D, et al. The role of neutrophils in the induction of glomerulonephritis by anti-myeloperoxidase antibodies. Am J Pathol 2005; 167: 39-45.

148. Xiao H, Schreiber A, Heeringa P, Falk RJ, et al. Alternative complement pathway in the pathogenesis of disease mediated by anti-neutrophil cytoplasmic autoantibodies. Am J Pathol 2007; 170: 52-64.

149. Wang Y, Rollins SA, Madri JA, Matis LA. Anti-C5 monoclonal antibody therapy prevents collagen-induced arthritis and ameliorates established disease. Proc Natl Acad Sci US A 1995; 92: 8955-8959.

150. Redl H, Dinges HP, Buurman WA, van der Linden CJ, et al. Expression of endothelial leukocyte adhesion molecule-1 in septic but not traumatic/hypovolemic shock in the baboon. Am J Pathol 1991; 139: 461-466.

151. Trouw LA, Seelen MA, Duijs JM, Benediktsson H, et al. Glomerular deposition of C1q and anti-C1q antibodies in mice following injection of antimouse C1q antibodies. Clin Exp Immunol 2003; 132: 32-39.

152. Wang Y, Hu Q, Madri JA, Rollins SA, et al. Amelioration of lupus-like autoimmune disease in NZB/WF1 mice after treatment with a blocking monoclonal antibody specific for complement component C5. Proc Natl Acad Sci U S A 1996; 93: 8563-8568.

153. Trendelenburg M, Fossati-Jimack L, Cortes-Hernandez J, Turnberg D, et al. The role of complement in cryoglobulin-induced immune complex glomerulonephritis. J Immunol 2005; 175: 6909-6914.

154. Haas M, Eustace JA. Immune complex deposits in ANCA-associated crescentic glomerulonephritis: a study of 126 cases. Kidney Int 2004; 65: 2145-2152.

155. Endo M, Ohi H, Ohsawa I, Fujita T, et al. Glomerular deposition of mannose-binding lectin (MBL) indicates a novel mechanism of complement activation in IgA nephropathy. Nephrol Dial Transplant 1998; 13: 1984-1990. 
156. Wirthmueller U, Dewald B, Thelen M, Schafer MK, et al. Properdin, a positive regulator of complement activation, is released from secondary granules of stimulated peripheral blood neutrophils. J Immunol 1997; 158: 4444-4451.

157. Mellbye OJ, Mollnes TE, Steen LS. IgG subclass distribution and complement activation ability of autoantibodies to neutrophil cytoplasmic antigens (ANCA). Clin Immunol Immunopathol 1994; 70: 32-39.

158. Ward PA, Hill JH. C5 chemotactic fragments produced by an enzyme in lysosomal granules of neutrophils. I Immunol 1970; 104: 535-543.

159. Jagels MA, Daffern PJ, Hugli TE. C3a and C5a enhance granulocyte adhesion to endothelial and epithelial cell monolayers: epithelial and endothelial priming is required for C3a-induced eosinophil adhesion. Immunopharmacology 2000; 46: 209-222.

160. DiScipio RG, Daffern PJ, Jagels MA, Broide DH, et al. A comparison of C3a and C5a-mediated stable adhesion of rolling eosinophils in postcapillary venules and transendothelial migration in vitro and in vivo. $J$ Immunol 1999; 162: 1127-1136.

161. Schwaeble WJ, Reid KB. Does properdin crosslink the cellular and the humoral immune response? Immunol Today 1999; 20: 17-21.

162. Muller Kobold AC, Kallenberg CG, Cohen Tervaert JW. Monocyte activation in patients with Wegener's granulomatosis. Ann Rheum Dis 1999; 58: 237-245.

163. Weidner S, Neupert W, Goppelt-Struebe M, Rupprecht HD. Antineutrophil cytoplasmic antibodies induce human monocytes to produce oxygen radicals in vitro. Arthritis Rheum 2001; 44: 1698-1706.

164. Rastaldi MP, Ferrario F, Crippa A, Dell'Antonio G, et al. Glomerular monocyte-macrophage features in ANCA-positive renal vasculitis and cryoglobulinemic nephritis. J Am Soc Nephrol 2000; 11: 2036-2043.

165. Weidner S, Carl M, Riess R, Rupprecht HD. Histologic analysis of renal leukocyte infiltration in antineutrophil cytoplasmic antibody-associated vasculitis: importance of monocyte and neutrophil infiltration in tissue damage. Arthritis Rheum 2004; 50: 3651-3657.

166. Ferrario F, Rastaldi MP. Necrotizing-crescentic glomerulonephritis in ANCA-associated vasculitis: the role of monocytes. Nephrol Dial Transplant 1999; 14: 1627-1631.

167. Sanders JS, Huitma MG, Kallenberg CG, Stegeman CA. Prediction of relapses in PR3-ANCA-associated vasculitis by assessing responses of ANCA titres to treatment. Rheumatology (Oxford) 2006; 45: 724-729.

168. Huugen D, Tervaert JW, Heeringa P. Antineutrophil cytoplasmic autoantibodies and pathophysiology: new insights from animal models. Curr Opin Rheumatol 2004; 16: 4-8.

169. Trouw LA, Groeneveld TW, Seelen MA, Duijs JM, et al. Anti-C1q autoantibodies deposit in glomeruli but are only pathogenic in combination with glomerular C1q-containing immune complexes. J Clin Invest 2004; 114: 679-688.

170. Kobayashi K, Shibata T, Sugisaki T. Aggravation of rat nephrotoxic serum nephritis by antimyeloperoxidase antibodies. Kidney Int 1995; 47: 454-463.

171. Ruth AJ, Kitching AR, Kwan RY, Odobasic D, et al. Anti-Neutrophil Cytoplasmic Antibodies and Effector CD4+ Cells Play Nonredundant Roles in Anti-Myeloperoxidase Crescentic Glomerulonephritis. J Am Soc Nephrol 2006; 17: 1940-1949.

172. Cambridge G, Leaker B, Hall TJ. Production and characterization of mouse monoclonal antibodies to native human myeloperoxidase. Hybridoma 1992; 11: 381-384.

173. Nachman PH, Reisner HM, Yang JJ, Jennette JC, et al. Shared idiotypy among patients with myeloperoxidase-anti-neutrophil cytoplasmic autoantibody associated glomerulonephritis and vasculitis. Lab Invest 1996; 74: 519-527.

174. Short AK, Lockwood CM. Studies of epitope restriction on myeloperoxidase (MPO), an important antigen in systemic vasculitis. Clin Exp Immunol 1997; 110: 270-276.

175. Fujii A, Tomizawa K, Arimura Y, Nagasawa T, et al. Epitope analysis of myeloperoxidase (MPO) specific anti-neutrophil cytoplasmic autoantibodies (ANCA) in MPO-ANCA-associated glomerulonephritis. Clin Nephrol 2000; 53: 242-252.

176. Erdbrugger U, Hellmark T, Bunch DO, Alcorta DA, et al. Mapping of myeloperoxidase epitopes recognized by MPO-ANCA using human-mouse MPO chimers. Kidney Int 2006; 69: 1799-1805.

177. van der Geld YM, Stegeman CA, Kallenberg CG. B cell epitope specificity in ANCA-associated vasculitis: does it matter? Clin Exp Immunol 2004; 137: 451-459.

178. Venturelli D, Bittenbender S, Rovera G. Sequence of the murine myeloperoxidase (MPO) gene. Nucleic Acids Res 1989; 17: 7987-7988.

179. Patry YC, Nachman PH, Audrain MA, Falk RJ, et al. Difference in antigenic determinant profiles between human and rat myeloperoxidase. Clin Exp Immunol 2003; 132: 505-508.

180. Stuart JM, Dixon FJ. Serum transfer of collagen-induced arthritis in mice. J Exp Med 1983; 158: 378-392.

181. Terato K, Harper DS, Griffiths MM, Hasty DL, et al. Collagen-induced arthritis in mice: synergistic effect of E. coli lipopolysaccharide bypasses epitope specificity in the induction of arthritis with monoclonal antibodies to type II collagen. Autoimmunity 1995; 22: 137-147. 
182. Maccioni M, Zeder-Lutz G, Huang H, Ebel C, et al. Arthritogenic monoclonal antibodies from K/BxN mice. J Exp Med 2002; 195: 1071-1077.

183. Amagai M, Tsunoda K, Suzuki H, Nishifuji K, et al. Use of autoantigen-knockout mice in developing an active autoimmune disease model for pemphigus. J Clin Invest 2000; 105: 625-631.

184. Tsunoda K, Ota T, Aoki M, Yamada T, et al. Induction of pemphigus phenotype by a mouse monoclonal antibody against the amino-terminal adhesive interface of desmoglein 3. J Immunol 2003; 170: 2170-2178.

185. Xiao H, Heeringa P, Falk RJ, Jennette JC. The Role of Fc gamma receptors in the induction of glomerulonephritis and pulmonary granulomatous inflammation in mice by anti-myeloperoxidase antibodies (anti-MPO)[abstract]. J Am Soc Nephrol 2004; 15: 37A.

186. Fossati-Jimack L, Ioan-Facsinay A, Reininger L, Chicheportiche Y, et al. Markedly different pathogenicity of four immunoglobulin $G$ isotype-switch variants of an antierythrocyte autoantibody is based on their capacity to interact in vivo with the low-affinity Fcgamma receptor III. J Exp Med 2000; 191: 1293-1302.

187. Huugen D, van Esch A, Xiao H, Peutz-Kootstra CJ, et al. Inhibition of complement factor C5 protects against anti-myeloperoxidase antibody-mediated glomerulonephritis in mice. Kidney Int. In press.

188. Azeredo da Silveira S, Kikuchi S, Fossati-Jimack L, Moll T, et al. Complement activation selectively potentiates the pathogenicity of the $\mathrm{IgG} 2 \mathrm{~b}$ and $\mathrm{IgG} 3$ isotypes of a high affinity anti-erythrocyte autoantibody. J Exp Med 2002; 195: 665-672.

189. Daemen MA, van de Ven MW, Heineman E, Buurman WA. Involvement of endogenous interleukin-10 and tumor necrosis factor-alpha in renal ischemia-reperfusion injury. Transplantation 1999; 67: 792-800.

190. Daemen MA, van 't Veer C, Wolfs TG, Buurman WA. Ischemia/reperfusion-induced IFN-gamma upregulation: involvement of IL-12 and IL-18. J Immunol 1999; 162: 5506-5510.

191. De Vries B, Matthijsen RA, Wolfs TG, Van Bijnen AA, et al. Inhibition of complement factor C5 protects against renal ischemia-reperfusion injury: inhibition of late apoptosis and inflammation. Transplantation 2003; 75: 375-382.

192. Nagra RM, Becher B, Tourtellotte WW, Antel JP, et al. Immunohistochemical and genetic evidence of myeloperoxidase involvement in multiple sclerosis. J Neuroimmunol 1997; 78: 97-107.

193. Ramsaransing G, Teelken A, Prokopenko VM, Arutjunyan AV, et al. Low leucocyte myeloperoxidase activity in patients with multiple sclerosis. J Neurol Neurosurg Psychiatry 2003; 74: 953-955.

194. Baldus S, Heeschen C, Meinertz T, Zeiher AM, et al. Myeloperoxidase serum levels predict risk in patients with acute coronary syndromes. Circulation 2003; 108: 1440-1445.

195. Hazen SL, Hsu FF, Duffin K, Heinecke JW. Molecular chlorine generated by the myeloperoxidasehydrogen peroxide-chloride system of phagocytes converts low density lipoprotein cholesterol into a family of chlorinated sterols. J Biol Chem 1996; 271: 23080-23088.

196. Hazell LJ, Arnold L, Flowers D, Waeg G, et al. Presence of hypochlorite-modified proteins in human atherosclerotic lesions. J Clin Invest 1996; 97: 1535-1544.

197. Podrez EA, Schmitt D, Hoff HF, Hazen SL. Myeloperoxidase-generated reactive nitrogen species convert LDL into an atherogenic form in vitro. J Clin Invest 1999; 103: 1547-1560.

198. Malle E, Waeg G, Schreiber R, Grone EF, et al. Immunohistochemical evidence for the myeloperoxidase/H2O2/halide system in human atherosclerotic lesions: colocalization of myeloperoxidase and hypochlorite-modified proteins. Eur J Biochem 2000; 267: 4495-4503.

199. Hazen SL, Gaut JP, Crowley JR, Hsu FF, et al. Elevated levels of protein-bound phydroxyphenylacetaldehyde, an amino-acid-derived aldehyde generated by myeloperoxidase, are present in human fatty streaks, intermediate lesions and advanced atherosclerotic lesions. Biochem J 2000; 352 Pt 3: 693-699.

200. Bergt C, Pennathur S, Fu X, Byun J, et al. The myeloperoxidase product hypochlorous acid oxidizes HDL in the human artery wall and impairs ABCA1-dependent cholesterol transport. Proc Natl Acad Sci U S A 2004; 101: 13032-13037.

201. de Vries B, Kohl J, Leclercq WK, Wolfs TG, et al. Complement factor C5a mediates renal ischemiareperfusion injury independent from neutrophils. J Immunol 2003; 170: 3883-3889.

202. Rabb H, Mendiola CC, Dietz J, Saba SR, et al. Role of CD11a and CD11b in ischemic acute renal failure in rats. Am J Physiol 1994; 267: F1052-1058.

203. Klausner JM, Paterson IS, Goldman G, Kobzik L, et al. Postischemic renal injury is mediated by neutrophils and leukotrienes. Am J Physiol 1989; 256: F794-802.

204. Paller MS. Effect of neutrophil depletion on ischemic renal injury in the rat. J Lab Clin Med 1989; 113: 379386.

205. Thornton MA, Winn R, Alpers CE, Zager RA. An evaluation of the neutrophil as a mediator of in vivo renal ischemic-reperfusion injury. Am J Pathol 1989; 135: 509-515.

206. Heinzelmann M, Mercer-Jones MA, Passmore JC. Neutrophils and renal failure. Am J Kidney Dis 1999; 34: 384-399. 
207. Kaushal GP, Basnakian AG, Shah SV. Apoptotic pathways in ischemic acute renal failure. Kidney Int 2004; 66: 500-506.

208. Dagher PC. Apoptosis in ischemic renal injury: roles of GTP depletion and p53. Kidney Int 2004; 66: 506509 .

209. Daemen MA, van 't Veer C, Denecker G, Heemskerk VH, et al. Inhibition of apoptosis induced by ischemia-reperfusion prevents inflammation. J Clin Invest 1999; 104: 541-549.

210. Kelly KJ, Plotkin Z, Dagher PC. Guanosine supplementation reduces apoptosis and protects renal function in the setting of ischemic injury. J Clin Invest 2001; 108: 1291-1298.

211. Kelly KJ, Plotkin Z, Vulgamott SL, Dagher PC. P53 mediates the apoptotic response to GTP depletion after renal ischemia-reperfusion: protective role of a p53 inhibitor. J Am Soc Nephrol 2003; 14: 128-138.

212. Zhou W, Farrar CA, Abe K, Pratt JR, et al. Predominant role for C5b-9 in renal ischemia/reperfusion injury. J Clin Invest 2000; 105: 1363-1371.

213. Askari AT, Brennan ML, Zhou X, Drinko J, et al. Myeloperoxidase and plasminogen activator inhibitor 1 play a central role in ventricular remodeling after myocardial infarction. J Exp Med 2003; 197: 615-624.

214. Vasilyev N, Williams T, Brennan ML, Unzek S, et al. Myeloperoxidase-generated oxidants modulate left ventricular remodeling but not infarct size after myocardial infarction. Circulation 2005; 112: 2812-2820.

215. Brennan ML, Anderson MM, Shih DM, Qu XD, et al. Increased atherosclerosis in myeloperoxidasedeficient mice. J Clin Invest 2001; 107: 419-430.

216. Brennan M, Gaur A, Pahuja A, Lusis AJ, et al. Mice lacking myeloperoxidase are more susceptible to experimental autoimmune encephalomyelitis. J Neuroimmunol 2001; 112: 97-105.

217. Takizawa S, Aratani Y, Fukuyama N, Maeda N, et al. Deficiency of myeloperoxidase increases infarct volume and nitrotyrosine formation in mouse brain. J Cereb Blood Flow Metab 2002; 22: 50-54.

218. Nauseef WM. The proper study of mankind. J Clin Invest 2001; 107: 401-403.

219. Jandl RC, Andre-Schwartz J, Borges-DuBois L, Kipnes RS, et al. Termination of the respiratory burst in human neutrophils. J Clin Invest 1978; 61: 1176-1185.

220. Robson MG, Cook HT, Pusey CD, Walport MJ, et al. Antibody-mediated glomerulonephritis in mice: the role of endotoxin, complement and genetic background. Clin Exp Immunol 2003; 133: 326-333.

221. Robson MG, Cook HT, Botto M, Taylor PR, et al. Accelerated nephrotoxic nephritis is exacerbated in C1qdeficient mice. J Immunol 2001; 166: 6820-6828.

222. Harboe M, Ulvund G, Vien L, Fung M, et al. The quantitative role of alternative pathway amplification in classical pathway induced terminal complement activation. Clin Exp Immunol 2004; 138: 439-446.

223. Riechmann L, Clark M, Waldmann H, Winter G. Reshaping human antibodies for therapy. Nature 1988; 332: 323-327.

224. Thomas TC, Rollins SA, Rother RP, Giannoni MA, et al. Inhibition of complement activity by humanized anti-C5 antibody and single-chain Fv. Mol Immunol 1996; 33: 1389-1401.

225. Hillmen P, Young NS, Schubert J, Brodsky RA, et al. The complement inhibitor eculizumab in paroxysmal nocturnal hemoglobinuria. N Engl J Med 2006; 355: 1233-1243.

226. Fitch JC, Rollins S, Matis L, Alford B, et al. Pharmacology and biological efficacy of a recombinant, humanized, single-chain antibody C5 complement inhibitor in patients undergoing coronary artery bypass graft surgery with cardiopulmonary bypass. Circulation 1999; 100: 2499-2506.

227. Spronk HMH, Huugen D, Frederix K, Loubele S, et al. A Hypercoagulable State in Myeloperoxidase Deficient Mice [Abstract]. J Thromb Haemost 2005; 3, S1: P1208.

228. Bekesi G, Heinle H, Kakucs R, Pazmany T, et al. Effect of inhibitors of myeloperoxidase on the development of aortic atherosclerosis in an animal model. Exp Gerontol 2005; 40: 199-208.

229. Tervaert JW, Popa ER, Bos NA. The role of superantigens in vasculitis. Curr Opin Rheumatol 1999; 11: 2433.

230. Hogan SL, Satterly KK, Dooley MA, Nachman PH, et al. Silica exposure in anti-neutrophil cytoplasmic autoantibody-associated glomerulonephritis and lupus nephritis. J Am Soc Nephrol 2001; 12: 134-142.

231. Lane SE, Watts RA, Bentham G, Innes NJ, et al. Are environmental factors important in primary systemic vasculitis? A case-control study. Arthritis Rheum 2003; 48: 814-823.

232. Rihova Z, Maixnerova D, Jancova E, Pelclova D, et al. Silica and asbestos exposure in ANCA-associated vasculitis with pulmonary involvement. Ren Fail 2005; 27: 605-608.

233. Beaudreuil S, Lasfargues G, Laueriere L, Ghoul ZE, et al. Occupational exposure in ANCA-positive patients: a case-control study. Kidney Int 2005; 67: 1961-1966.

234. Tervaert JW, Stegeman CA, Kallenberg CG. Silicon exposure and vasculitis. Curr Opin Rheumatol 1998; 10: $12-17$.

235. Kruger T, Benke D, Eitner F, Lang A, et al. Identification and functional characterization of dendritic cells in the healthy murine kidney and in experimental glomerulonephritis. J Am Soc Nephrol 2004; 15: 613-621. 
236. Harper L, Ren Y, Savill J, Adu D, et al. Antineutrophil cytoplasmic antibodies induce reactive oxygendependent dysregulation of primed neutrophil apoptosis and clearance by macrophages. Am J Pathol 2000; 157: 211-220.

237. Gallucci S, Lolkema M, Matzinger P. Natural adjuvants: endogenous activators of dendritic cells. Nat Med 1999; 5: 1249-1255.

238. Eriksson U, Ricci R, Hunziker L, Kurrer MO, et al. Dendritic cell-induced autoimmune heart failure requires cooperation between adaptive and innate immunity. Nat Med 2003; 9: 1484-1490.

239. Ludewig B, Odermatt B, Landmann S, Hengartner H, et al. Dendritic cells induce autoimmune diabetes and maintain disease via de novo formation of local lymphoid tissue. J Exp Med 1998; 188: 1493-1501.

240. Armengol MP, Juan M, Lucas-Martin A, Fernandez-Figueras MT, et al. Thyroid autoimmune disease: demonstration of thyroid antigen-specific B cells and recombination-activating gene expression in chemokine-containing active intrathyroidal germinal centers. Am J Pathol 2001; 159: 861-873.

241. Voswinkel J, Muller A, Lamprecht P. Is PR3-ANCA Formation Initiated in Wegener's Granulomatosis Lesions? Granulomas as Potential Lymphoid Tissue Maintaining Autoantibody Production. Ann N Y Acad Sci 2005; 1051: 12-19.

242. Hoffman GS, Sechler JM, Gallin JI, Shelhamer JH, et al. Bronchoalveolar lavage analysis in Wegener's granulomatosis. A method to study disease pathogenesis. Am Rev Respir Dis 1991; 143: 401-407. 



\section{Nederlandse samenvatting}

De ziekte van Wegener, microscopische angiitis, het syndroom van Churg-Strauss en "pauciimmune rapidly progressive crescentic glomerulonephritis", een vorm van glomerulonefritis die wordt gekenmerkt door zijn snelle progressie en het vrijwel afwezig zijn van immuuncomplexen, zijn ziekten die gekenmerkt worden door steriele inflammatie van met name de kleine bloedvaten. De organen die het meest frequent aangetast zijn, zijn de nieren, de longen, en het KNO-gebied, maar feitelijk kan de ziekte in ieder orgaan optreden. Het beloop kan zeer ernstig zijn, en leiden tot ernstig disfunctioneren van het getroffen orgaan. De huidige standaardtherapie bestaat uit diverse middelen die alle op een vrij aspecifieke manier de afweer onderdrukken. De effectiviteit ervan schiet frequent tekort, en bovendien kan de behandeling gepaard gaan met ernstige bijwerkingen.

Een fenomeen dat deze "kleine vaten-vasculitiden" met elkaar gemeen hebben, is hun associatie met de aanwezigheid van anti-neutrofiel cytoplasmatische autoantilichamen (ANCA). Zoals de naam al suggereert, zijn dit antilichamen die lichaamseigen antigenen herkennen, in het bijzonder eiwitten die zich bevinden in het cytoplasma van neutrofiele granulocyten. Hoewel de antilichamen gericht kunnen zijn tegen diverse eiwitten, zijn ANCA in de meeste patiënten met bovenstaande ziekten specifiek voor proteïnase 3 (PR3) of myeloperoxidase (MPO).

Tot voor kort bestond tussen de aanwezigheid van ANCA en vasculitis alleen een associatie, en kon in vivo niet aangetoond worden dat de autoantilichamen ook daadwerkelijk vasculitis en glomerulonefritis veroorzaakten. In 2002 is echter aangetoond dat muizen waarbij antilichamen gericht tegen muisMPO worden ingespoten vasculitis ontwikkelen, die wordt gekenmerkt door de aanwezigheid van rode en witte bloedcellen en eiwit in de urine, tekenen van nierfunctiestoornissen in het bloed en ontstekingen in de nieren en longen, die erg lijken op de afwijkingen die gevonden worden bij mensen met (MPO-) ANCA geassocieerde vasculitis. Deze ontdekking wierp niet alleen een geheel nieuw licht op het ontstaan van deze ziekte, maar creëerde ook de mogelijkheid om in dit muismodel andere factoren te onderzoeken die mogelijk betrokken zijn bij de pathogenese, en om potentiële nieuwe therapieën te testen. In dit proefschrift worden experimenten beschreven -vooral uitgevoerd in het hierboven beschreven muismodel- , waarmee we wilden uitzoeken waarom anti-MPO antilichamen glomerulonefritis veroorzaken in muizen, welke factoren daarbij betrokken zijn, en hoe we dit kunnen beïnvloeden. We denken dat deze muizen een goed model zijn voor de pathogenese van MPO-ANCA geassocieerde glomerulonefritis in mensen; het uiteindelijke doel is dan ook om het ontstaan van 
deze ziekte bij mensen beter te begrijpen, en een therapie te ontdekken die beter (dat wil zeggen, effectiever, en met minder bijwerkingen gepaard gaand) werkt dan de huidige behandeling.

In hoofdstuk 1 wordt een introductie gegeven over ANCA-geassocieerde vasculitiden, in het bijzonder MPO-ANCA geassocieerde vasculitis en glomerulonefritis. Tevens worden kort een aantal andere diermodellen van ANCA-geassocieerde ziekte besproken, aan de hand van hun plus- en minpunten. Uitgebreider wordt ingegaan op het hierboven beschreven muismodel, waarin het merendeel van onze studies zijn verricht.

In hoofdstuk 2 wordt beschreven wat de rol is van proinflammatoire stimuli in het muismodel van anti-MPO antilichaamgeïnduceerde glomerulonephritis. Er zijn een aantal redenen om te veronderstellen dat ontstekingseiwitten belangrijk zijn in het ontstaan van MPO-ANCA geassocieerde ziekte bij mensen, en in deze studie tonen we aan dat dit in ieder geval opgaat voor muizen. Bovendien laten we zien dat het tevoren inactiveren van één van die eiwitten -tumor necrosis factor-alfa, TNF $\alpha$ - leidt tot significant minder anti-MPO antilichaamgeïnduceerde nierschade, maar de ziekte niet helemaal voorkómt. Dit heeft implicaties voor het potentiële nut van anti-TNF $\alpha$ behandeling van mensen met ANCA-geassocieerde ziekte, een onderwerp dat verder wordt uitgewerkt in hoofdstuk 3. De conclusie van deze uitgebreide review van in vitro, in vivo, en klinische studies naar de rol van TNF $\alpha$ (/-inhibitie) in ANCA-geassocieerde vasculitis, is dat ook deze behandeling niet altijd voldoende effectief is, en nog met veel bijwerkingen gepaard gaat.

Hoofdstuk 4 behandelt de rol van het complementsysteem in het muismodel van anti-MPO antilichaamgeïnduceerde glomerulonefritis. Dit is een groep van eiwitten die samen belangrijk zijn voor de verdediging van het lichaam tegen schadelijke invloeden. Het blijkt echter dat sommige onderdelen van het complementsysteem ook een dermate belangrijke plaats hebben in het ontstaan van ziekte in het muismodel, dat deficiëntie ervan -hetzij in genetisch veranderde muizen, hetzij ten gevolge van het inspuiten van de muizen met een "complement-remmer"- het ontstaan van ziekte voorkómt. De meest interessante ontdekking uit onze studie was dat het tevoren inactiveren van één specifiek eiwit van het complementsysteem (factor C5) ziekte niet alleen kan voorkómen, maar dat het inspuiten van muizen die al ziek zijn met een factor C5remmer ook leidt tot een reductie van anti-MPO antilichaamgeïnduceerde nierschade van meer dan $80 \%$. Aangezien er al middelen bestaan die factor C5 in mensen kan remmen, heeft deze ontdekking mogelijk belangrijke consequenties voor de behandeling van mensen met MPOANCA geassocieerde vasculitis in de toekomst. 
In hoofdstuk 5 beschrijven we de ontwikkeling van een set muisMPO-specifieke monoklonale (dat wil zeggen, slechts bindend aan één specifiek stukje van het MPO-molekuul) antilichamen. Doel hiervan was te onderzoeken of er een bepaald type anti-MPO antilichaam verantwoordelijk is voor het ontstaan van ziekte. Echter, hoewel we elf MPO-specifieke antilichamen hebben gegenereerd, bleek geen enkele daarvan op zichzelf of in combinatie met elkaar ziekte te veroorzaken in muizen. Wel bleken sommige van de antilichamen in staat om een milde vorm van glomerulonefritis, geïnduceerd met antilichamen gericht tegen de glomerulaire basaalmembraan, te verhevigen. De betekenis hiervan voor de humane ANCA-geassocieerde vasculitis is nog niet helemaal duidelijk; in de toekomst zullen de verkregen antilichamen mogelijk van nut zijn bij verder onderzoek naar subtypespecifieke effecten van antilichamen in autoimmuunziekten.

Behalve het antigeen waar MPO-ANCA specifiek voor zijn, is MPO ook een zeer veelzijdig neutrofiel eiwit, met bepaalde eigenschappen en functies. Hoewel er weinig onderzoek naar is gedaan, bestaan er wel indirecte aanwijzingen dat MPO zelf een rol speel $t$ in de pathogenese van MPO-ANCA geassocieerde vasculitis. Omdat het niet mogelijk is dit uit te zoeken in ons muismodel, proberen we in hoofdstuk 6 de eigenschappen van MPO nader te onderzoeken in een muismodel van renale ischemie-reperfusieschade. Dit is een model waarin kan worden onderzocht hoe zuurstofgebrek in de nier, gevolgd door het herstel van de circulatie (zoals dat bijvoorbeeld optreedt bij een niertransplantatie) tot schade leidt. Het blijkt dat de afwezigheid van MPO muizen beschermt tegen renale schade geïnduceerd door ischemie gevolgd door reperfusie. Mogelijk oefent MPO dus ter plaatse van een ontsteking een toxisch effect uit op de omgeving. Het is mogelijk dat iets dergelijks ook een rol speelt bij (MPO-) ANCA geassocieerde glomerulonefritis, maar onze resultaten staan in opvallend contrast met studies waarin MPOdeficiëntie juist leidt tot meer schade na de inductie van bijvoorbeeld atherosclerose of een bepaalde vorm van encefalitis.

In hoofdstuk 7 worden de voorgaande hoofdstukken nogmaals in een ruimer perspectief besproken, en wordt kort ingegaan op de vraag hoe het menselijk lichaam mogelijk wordt aangezet tot de productie van ANCA. 



\section{Dankwoord}

$\mathrm{Nu}$ ook het feest vastere vormen aan begint te nemen, wordt het tijd om me bezig te gaan houden met de meest verantwoordelijke taak van een promovendus: het schrijven van een dankwoord dat íedereen behelst die -op directe of meer indirecte wijze- een bijdrage aan dit proefschrift heeft geleverd.

Peter, een goed proefschrift is afhankelijk van de uitvoering, maar vooral ook van de kwaliteit van het project en de begeleiding. Ik hoop dat dit proefschrift ook voor jou aantoont dat deze ingrediënten in meer dan voldoende mate aanwezig waren in onze samenwerking. Ik wil je bij deze bedanken voor de leerzame en gezellige tijd die ik onder jouw hoede heb gehad. Tenslotte wens ik je veel succes toe in Groningen, en ik hoop dat we contact houden. Jan Willem, bedankt voor het vertrouwen, en voor het mede in het oog houden van de grote lijnen. Onze discussies hielpen me realiseren dat er niet alleen muizen, maar ook mensen met ANCA-gemedieerde vasculitis bestaan!

Anita, zonder jouw ervaring, kwaliteiten, energie, toewijding, mentale steun, gemopper, en niet in het minst je vrijdagse kookkunsten, zou dit boekje waarschijnlijk nog niet half zo dik zijn geweest. Ons project vereiste een hoge mate van teamwork, en ik denk dat niemand méér geschikt was voor ons team dan jij. Bedankt dat je de samenwerking hebt willen afronden door mijn paranimf te zijn. En ik kan me heel goed voorstellen dat jullie je eerste dochter niet ELISA hebben genoemd!

De lijst van collega's die verder betrokken zijn geweest bij het onderzoek en bij de vele interessante zijpaadjes die we zijn ingeslagen lijkt eindeloos, en ik hoop dat ik de mensen die ik nu ongetwijfeld vergeet niet te hard voor het hoofd stoot. Het enthousiasme waarmee iedereen heeft geholpen was indrukwekkend, en daar ben ik iedereen erg dankbaar voor. Een bijzonder woord van dank aan Bram, Jan, Ad, Pieter, Anita, de rest van het secretariaat, het lab Algemene Immunologie, José, Maria (ik ben je enorme steun tijdens de laatste maanden niet vergeten!), Petra, Ruud, Luciënne, Kim, Henk, Marcella, Marjan, Marjan, de mensen van het PLAN, Menno, Anique, Anna, Vicky, Bart, Robert, Astrid, Gerard, Wilfred, Heinz, Silvie, Ariane, Mariska, Carine, Saskia, Nadine, Paulien: jullie hebben mijn jaren als aio allemaal op je eigen manier beïnvloed, waren nooit te beroerd om een stapje extra te zetten (soms meer stapjes als dat eens nodig was ...), en/of mij met allerhande adviezen bij te staan. Eric Steenbergen, een speciaal woord van dank voor jou en je groep: zonder jullie hulp zou de beginfase van het project er een 
stuk magerder uitgezien hebben! Moh Daha, bedankt voor de zinvolle discussies en suggesties. Helaas hebben we niet alle plannen in de praktijk kunnen brengen, maar een gesprek met $u$ leverde altijd weer een geheel nieuwe blik op de materie. Tom, bedankt voor de diverse keren dat je ons controlecoupes hebt gestuurd. Finally, I would like to thank Professor Jennette and Hong Xiao for the inspiring and fruitful collaboration with their group.

Ik ben ervan overtuigd dat relativeringsvermogen in het leven van een promovendus onmisbaar is. Gelukkig was er de afgelopen periode een grote hoeveelheid vrienden beschikbaar om dit in de praktijk te brengen, om frustraties te delen, een biertje te drinken, wat te sporten, wat te eten, of gewoon maar wat te vertellen. Arthur, als er iemand was die me altijd begreep was jij het wel. We spreken elkaar de laatste tijd wat minder frequent, maar keer op keer blijkt dat dit de kwaliteit van het contact niet beïnvloedt. Koen, na onze gemeenschappelijke middelbare schooltijd werd de (geografische) afstand tussen ons eerst een stuk groter, maar de laatste paar jaar zijn onze paden op miraculeuze wijze weer steeds meer parallel gaan lopen. Bedankt voor alle gesprekken, het mij ertoe overhalen toch nog enige lichamelijke arbeid te verrichten in de vorm van een aantal halsbrekende potjes squash, en niet te vergeten je hulp de afgelopen tijd met alle promotiezaken. Ik ben dankbaar dat je mijn paranimf wil zijn.

Martijn en Steven, jullie bleken al snel meer dan zomaar twee collega-aio's. Jullie grenzeloze relativeringsvermogen, humor, betrokkenheid, enthousiasme, energie, en natuurlijk het door jullie geïnitieerde en (zeker na de komst van Twan) al snel legendarische "kook-klupje", leverden de broodnodige afleiding, en de energie om er de volgende dag weer met frisse moed tegenaan te gaan. Martijn, ik ben blij dat je mijn collega bent gebleven; ik ben ervan overtuigd dat je uiteindelijk je doel zult bereiken, en wens je veel succes op het pad ernaartoe.

Matthias, Daniëlle, Jeroen, Judith, Linda, Natasja, Kitty, Suzanne, Marjo, Jeroen, Jeroen, Maura, Dorien, Frank, Jaap, Joost, Nicole, Rianne: de meesten van jullie waren minder bij het project zelf betrokken, maar jullie wisten altijd voor de broodnodige ontspanning te zorgen! Joost, leuk dat je ook in het onderzoek bent gestapt!

Pap, mam, jullie hebben me áltijd, en in álles wat ik me iedere keer weer in mijn hoofd haalde, gesteund. Jullie enthousiasme, geduld en begrip hebben me geholpen te worden wie ik nu ben, en dit boekje is daar mede een gevolg van. Bedankt voor alles. 
Babs en Nienke, het is een drukke tijd geweest, en af en toe dreigden jullie daar de dupe van de worden. Gelukkig maakten jullie me daar altijd attent op voordat de boel uit de hand liep. Dank voor jullie "down to earth" zijn, en voor jullie steun bij alles.

Nadiya, your coming into my life was the greatest and most unexpected result of my time as a $\mathrm{PhD}$-student. You were the one missing piece in the jigsaw of my life; thank you for all your support, your warmth, your enthusiasm, and for being who you are. We have been dreaming together now for almost three years and I hope that we will complement each other for many more. 


\section{Curriculum vitae}

Dennis Huugen werd geboren in Eindhoven op 10 augustus 1976.

\section{Opleiding}

1988-1994 Gymnasium; Eindhovens Protestants Lyceum, Eindhoven

1994-1995 Psychologie; Radbout Universiteit Nijmegen, Nijmegen

1995-2001 Geneeskunde; Universiteit Maastricht, Maastricht

2001-2002 AGNIO Interne Geneeskunde; St. Elisabeth Ziekenhuis, Tilburg

2002-2006 AIO; Afdeling Interne Geneeskunde, Divisie Immunologie, Universiteit Maastricht, Maastricht

2006-heden AIOS Interne Geneeskunde; Academisch Ziekenhuis Maastricht, Maastricht

\section{Extracurriculair}

2003-2006 Bestuurslid namens Maastricht van het Platform Aio’s Nefrologie (PLAN) 


\section{Publications}

1. Huugen D, Schouten HC, Bos GM. Advantages and limitations of (non-) myeloablative allogeneic stem cell transplantation. Neth J Med 2002; 60: 162-169.

2. Huugen D, Bos GM, Jansen M, Lalisang R, et al. Non-myeloablative allogeneic stem cell transplantation in patients with solid tumours and patients with a haematological malignancy. Neth J Med 2002; 60: 170-173.

3. Huugen D, Tervaert JW, Heeringa P. Antineutrophil cytoplasmic autoantibodies and pathophysiology: new insights from animal models. Curr Opin Rheumatol 2004; 16: 4-8.

4. Xiao H, Heeringa P, Liu Z, Huugen D, et al. The role of neutrophils in the induction of glomerulonephritis by anti-myeloperoxidase antibodies. Am J Pathol 2005; 167: 39-45.

5. Huugen D, Xiao H, van Esch A, Falk RJ, et al. Aggravation of Anti-Myeloperoxidase Antibody-Induced Glomerulonephritis by Bacterial Lipopolysaccharide: Role of Tumor Necrosis Factor- $\alpha$. Am J Pathol 2005; 167: 47-58.

6. Heeringa P, Huugen D, Tervaert JW. Anti-neutrophil cytoplasmic autoantibodies and leukocyte-endothelial interactions: a sticky connection? Trends Immunol 2005; 26: 561-564.

7. Huugen D, Cohen Tervaert JW, Heeringa P. TNF-a bioactivity-inhibiting therapy in ANCA-associated vasculitis: clinical and experimental considerations. Clin J Am Soc Nephrol 2006; 1: 1100-1107.

8. Huugen D, van Esch A, Xiao H, Peutz-Kootstra CJ, et al. Inhibition of complement factor C5 protects against anti-myeloperoxidase antibody-mediated glomerulonephritis in mice. Kidney Int 2007; 71: 646-654. 
